Canadian

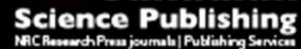

Canadian Journal of Microbiology Revue canadienne de de microbiologie

\title{
Synthetic cystic fibrosis sputum medium diminishes Burkholderia cenocepacia antifungal activity against Aspergillus fumigatus independently of phenylacetic acid production
}

\begin{tabular}{|r|l|}
\hline Journal: & Canadian Journal of Microbiology \\
\hline Manuscript ID & cjm-2016-0705.R1 \\
\hline Manuscript Type: & Article \\
\hline Date Submitted by the Author: & $13-J a n-2017$ \\
\hline Complete List of Authors: & $\begin{array}{l}\text { Lightly, Tasia Joy; University of Manitoba, Microbiology } \\
\text { Phung, Ryan R.; University of Manitoba, Microbiology } \\
\text { Sorensen, John L.; University of Manitoba, Chemistry } \\
\text { Cardona, Silvia T.; University of Manitoba, Microbiology; University of } \\
\text { Manitoba, Medical Microbiology \& Infectious Disease }\end{array}$ \\
\hline Keyword: & $\begin{array}{l}\text { phenylacetic acid, <i>Burkholderia cenocepacia</i>, <i>Aspergillus } \\
\text { fumigatus</i>, <i>Pseudomonas aeruginosa }</ i>, \text { cystic fibrosis }\end{array}$ \\
\hline &
\end{tabular}


Synthetic cystic fibrosis sputum medium diminishes Burkholderia cenocepacia antifungal activity against Aspergillus fumigatus independently of phenylacetic acid production

\footnotetext{
Tasia Joy Lightly ${ }^{1}$, Ryan R. Phung ${ }^{1}$, John L. Sorensen ${ }^{3}$ and Silvia T. Cardona ${ }^{1,2} *$

${ }^{1}$ Department of Microbiology, University of Manitoba, Winnipeg, Canada

${ }^{2}$ Department of Medical Microbiology \& Infectious Disease, University of Manitoba, Winnipeg, Canada

${ }^{3}$ Department of Chemistry, University of Manitoba, Winnipeg, Canada

*Corresponding author.

Mailing address: Silvia T. Cardona, Department of Microbiology, Buller Building, Room 213, University of Manitoba, Winnipeg, Manitoba, Canada R3T 2N2.

Phone: (204) 474-8997

Fax: (204) 474-7603

E-mail: Silvia.Cardona@umanitoba.ca
} 


\begin{abstract}
Phenylacetic acid (PAA), an intermediate of phenylalanine degradation, is emerging as a signal molecule in microbial interactions with the host. In this work, we explore the presence of phenylalanine and PAA catabolism in three microbial pathogens of the cystic fibrosis (CF) lung microbiome: Pseudomonas aeruginosa, Burkholderia cenocepacia and Aspergillus fumigatus. While in silico analysis of B. cenocepacia $\mathrm{J} 2315$ and A. fumigatus Af293 genome sequences showed complete pathways from phenylalanine to PAA, the $P$. aeruginosa PAO1 genome lacked several coding genes for phenylalanine and PAA catabolic enzymes. HPLC analysis of supernatants from B. cenocepacia K56-2 detected PAA when grown in LB but not in synthetic cystic fibrosis sputum medium (SCFM). However, we were unable to identify PAA production by A. fumigatus or P. aeruginosa in any of the conditions tested. The inhibitory effect of B. cenocepacia on A. fumigatus growth was evaluated using agar plate interaction assays. Inhibition of fungal growth by B. cenocepacia was lessened in SCFM but this effect was not dependent on bacterial production of PAA. In summary, while we demonstrated PAA production by $B$. cenocepacia, we were not able to link this metabolite with the $B$. cenocepacia- $A$. fumigatus microbial interaction in CF nutritional conditions.
\end{abstract}

Key Words phenylacetic acid, Burkholderia cenocepacia, Aspergillus fumigatus, Pseudomonas aeruginosa, cystic fibrosis 


\section{Introduction}

Cystic fibrosis (CF) is a human genetic disease characterized by mutations in the cystic fibrosis transmembrane conductance regulator (CFTR) gene (Cheng et al. 1990; Riordan et al. 1989). CFTR malfunction results in an imbalance of electrolytes and water in the airways, leading to accumulation of thick dehydrated mucus in the patient's lungs (Matsui et al. 1998). The CF mucus is rich in nutrients, such as amino acids (Palmer et al. 2007), and supports the establishment of a polymicrobial community that leads to lung inflammation, chronic infection, and loss of lung function (Hauser et al. 2011).

Haemophilus influenzae and Staphylococcus aureus are generally the first to colonize the CF lung, followed by Pseudomonas aeruginosa and, as the disease progresses, members of the Burkholderia cepacia complex (Bcc) (Lipuma 2010; Mahenthiralingam et al. 2005). Although $P$. aeruginosa is the most common opportunistic pathogen in $\mathrm{CF}$ patients, the outcomes of Bcc infection are the most severe (Courtney et al. 2004; Courtney et al. 2007). Bacteria are not the only colonizers of the CF lung. Filamentous fungi, such as Aspergillus fumigatus, are very prevalent (Bakare et al. 2003; Mortensen et al. 2011; Paugam et al. 2010). While research and treatment of CF patients have traditionally focused on individual pathogens, there is accumulating evidence that the progression of CF lung disease is governed by the complex interactions of the $\mathrm{CF}$ polymicrobial community (Sibley et al. 2006; Sibley and Surette 2011). Of particular interest is the possibility of microbial beneficial interactions that may result in poorer outcomes for the patient.

Microbial production of phenylacetic acid (PAA) is emerging as a regulatory mechanism by which microorganisms may modulate their pathogenicity and interactions with the 
host. PAA produced by a paaABCDE mutant of B. cenocepacia decreased luxI/luxR-type QS signaling. The paaABCDE mutant was defective in QS-related virulence as demonstrated in a Caenorhabditis elegans model of infection (Pribytkova et al. 2014). In line with our findings, exogenous addition of PAA inhibited QS-regulated virulence traits of $P$. aeruginosa $\mathrm{PAO}$, such as pyocyanin and exopolysaccharide production, protease and elastase activity, and swimming motility (Musthafa et al. 2012). Intriguingly, Wang et al. found that $P$. aeruginosa PAO1 produced PAA at high cell densities in LB, which in turn attenuated P. aeruginosa's cytotoxicity (Wang et al. 2013). The ability of two prominent CF pathogens to use PAA to modify their QS-regulated virulence presents intriguing possibilities for a physiological role of PAA metabolism in microbial interactions as microbial production of PAA may result in intra- and interspecies regulation of virulence. This has been demonstrated in the rhizosphere where PAA, released as a phytotoxin by the root infecting fungus Rhizoctonia solani, can repress the biosynthesis of nematicidal compounds by Pseudomonas fluorescens, indirectly protecting the plant-parasitic nematode Meloidogyne incognita from biocontrol by $P$. fluorescens (Siddiqui and Shaukat 2004). PAA can also affect the host immune system directly. PAA excreted by Bacillus fortis can activate the induced systemic response of tomato plants, protecting the plants by priming them to respond to the plant pathogen responsible for Fusarium wilt disease (Akram et al. 2016). In zebrafish, PAA released by a mutant of Acinetobacter baumannii acted as a chemoattractant for neutrophils resulting in improved bacterial clearance (Bhuiyan et al. 2016). Understanding the links between bacterial metabolism, pathogenicity and host immune response may help in the development of treatments. 
In Escherichia coli and Pseudomonas putida, PAA can be produced aerobically from phenylalanine (Supplemental Fig. S1 and S2), according to the Kyoto Encyclopedia of Genes and Genomes (KEGG) pathway database (Kanehisa and Goto 2000; Kanehisa et al. 2016) and accumulated experimental evidence (Teufel et al. 2010; Teufel et al. 2011). Phenylalanine can be converted to PAA through 2-phenylacetamide, which is then transformed to PAA by an amidase. Conversion of phenylalanine to PAA can also occur through synthesis of phenylethylamine by an aromatic-L-amino-acid decarboxylase followed by conversion to phenylacetaldehyde by a primary amine oxidase, such as the TynA (MaoA) enzyme of E. coli (Steinebach et al. 1996). Finally, phenylacetaldehyde is converted to PAA by a phenylacetaldehyde dehydrogenase, FeaB (PadA) (Ferrandez et al. 1997). In turn, PAA can be further degraded to succinyl-CoA and acetyl-CoA in aerobic conditions. In E. coli and P. putida (Supplemental Fig. S1 and S2), PAA is first converted to phenylacetyl-coenzyme A (PAA-CoA) by a CoA ligase, PaaK (MartinezBlanco et al. 1990; Vitovski 1993). Next, the aromatic ring of PAA-CoA undergoes epoxidation by PaABC(D)E, a four or five subunit-monoxygenase complex (Teufel et al. 2010; Teufel et al. 2012). Then, PaaG isomerizes the epoxide into an oxepin and the bifunctional enzyme PaaZ performs the hydrolysis of the oxepin and the subsequent oxidation of the terminal aldehyde group (Grishin and Cygler 2015; Teufel et al. 2010). The final steps of the PAA catabolic pathway, often referred to as the PAA catabolic lower pathway, are similar to the fatty acid $\beta$-degradation pathway. The product of PaaZ is cleaved by a CoA thiolase PaaJ, rearranged by the CoA isomerase PaaG, and hydrated by PaaF to form 3-hydroxyadipyl-CoA. This intermediate is then oxidized by PaaH, 
before finally being converted to acetyl-CoA and succinyl-CoA by PaaJ (Grishin and Cygler 2015; Grishin and Cygler 2015; Nogales et al. 2007; Teufel et al. 2010).

The aim of this study was to examine three CF pathogens, B. cenocepacia, P. aeruginosa and A. fumigatus, for their ability to produce PAA in aerobic conditions by analyzing the presence of putative genes of phenylalanine and PAA catabolic pathways, the presence of PAA in culture supernatants, and the role of PAA in their interactions. We demonstrate that B. cenocepacia is able to release PAA when grown in LB medium. The antifungal activity of both $B$. cenocepacia and $P$. aeruginosa against $A$. fumigatus was decreased in conditions that mimic the nutrients of the CF sputum. However, these interactions were not mediated by bacterial PAA production.

\section{Materials and methods}

Strains, media preparation and growth conditions

Bacterial and fungal strains are listed in Table 1. Bacterial strains were grown overnight at $37^{\circ} \mathrm{C}$ in Luria-Bertani (LB) medium, unless otherwise specified. A. fumigatus UAMH 2978 was grown and maintained on potato dextrose agar (PDA) media at $37^{\circ} \mathrm{C}$. The growth of bacterial cultures was measured by optical density at $600 \mathrm{~nm}\left(\mathrm{OD}_{600}\right)$ using a Biotek Synergy 2 plate reader with values converted to $1-\mathrm{cm}-$ path-length $\mathrm{OD}_{600}$ by prior calibration with a GeneQuant $^{\mathrm{TM}}$ III 4283, version 4283V1.6. Synthetic cystic fibrosis sputum medium (SCFM) (Palmer et al. 2007) agar plates were made using 2X SCFM pre-warmed to $55^{\circ} \mathrm{C}$ and mixed in equal volumes with $3 \%$ agar cooled to $55^{\circ} \mathrm{C}$ for a final concentration of $1 \mathrm{X}$ SCFM and $1.5 \%$ agar. 
Computational analysis of the phenylalanine catabolic pathway in CF pathogens The organism-specific pathway maps for phenylalanine catabolism to PAA for E. coli K12 MG1655 (Supplemental Fig. S1 and Supplemental File S1) and P. putida KT2440 (Supplemental Fig. S2 and Supplemental File S1) were retrieved from the KEGG pathway database. Gene annotations were examined by performing bidirectional BLASTP searches using the BLAST server tool from NCBI (http://blast.ncbi.nlm.nih.gov/Blast.cgi). All BLASTP searches used the non-redundant protein sequences database (nr) and an expect threshold of $10^{-8}$. All other algorithm parameters used were the default settings.

Next, the genes in the organism-specific pathway map of B. cenocepacia J2315, $P$. aeruginosa PAO1, and A. fumigatus Af293 were analyzed using KEGG. For the steps with no gene product assigned, the amino acid sequences of the corresponding genes from P. putida KT2440 and E. coli K-12 MG1655 were used as queries for a bidirectional BLASTP search against either B. cenocepacia J2315, P. aeruginosa PAO1, or A. fumigatus Af293. Genes that were identified as hits by bidirectional BLASTP searches against either $P$. putida KT2440 or E. coli K-12 MG1655 were considered as putative coding genes for the corresponding enzymes. Examination of bacterial supernatants for the release of PAA in LB media Using the method previously described (Pribytkova et al. 2014), cultures were grown overnight on LB agar and a full loopful was inoculated into $100 \mathrm{~mL}$ of LB in $250 \mathrm{~mL}$ flasks. Flasks were incubated at $37^{\circ} \mathrm{C}$ with agitation at $230 \mathrm{rpm}$ for 24 hours and cells were pelleted at 7,000 rpm for 10 minutes. Supernatants were filtered through a $0.2 \mu \mathrm{m}$ vacuum filter (Sarstedt, Montréal, Québec) and stored at $4^{\circ} \mathrm{C}$ overnight before extraction. 
Supernatants were acidified to a $\mathrm{pH}$ of $\leq 2$ with hydrochloric acid (HCl) and extracted twice with $50 \mathrm{ml}$ of ethyl acetate $(100 \mathrm{ml}$ total). The organic phases were combined and sodium sulphate was added in excess. The organic phase was filtered using Whatman No. 1 filter paper and evaporated under reduced pressure. The crude extract was dissolved in methanol to a concentration of approximately $2 \mathrm{mg} / \mathrm{ml}$. HPLC vials were filled in duplicate with $1 \mathrm{ml}$ of suspended crude extract and evaporated under reduced pressure. Two mg of crude extract was dissolved in either $1 \mathrm{ml}$ of acidified methanol (1 $\mathrm{ml}$ of $\mathrm{HCl}$ in $50 \mathrm{ml}$ of methanol), for esterification, or $1 \mathrm{ml}$ of neutral methanol (no acid added) and heated for 15 minutes at $50^{\circ} \mathrm{C}$. Samples were cooled and analyzed by HPLC using a Waters HPLC Separation Module 2695, combined with PDA Detector Model 2996 and a $\mu$ Bondapak $^{\mathrm{TM}}$ Waters $\mathrm{C}_{18}(3.9 \times 300 \mathrm{~mm})$ column particle diameter of $15-20 \mu \mathrm{m}$, with $125 \AA$ pores. Several gradients and solvents were tested for optimal peak separation of extracted supernatants. For LB media extracts the solvents chosen were methanol and $0.075 \%$ aqueous trifluoracetic acid. The gradient was held at $20 \%$ methanol in $0.075 \%$ aqueous trifluoracetic acid for 10 minutes, increased to $100 \%$ methanol over 10 minutes following a linear gradient and held there for 20 minutes. Methanol was decreased to $20 \%$ using a linear gradient over 10 minutes and then held for 10 minutes. The total run time was 60 minutes with a flow rate of $1 \mathrm{~mL} / \mathrm{minute}$. The eluent was monitored continuously at 210-600 nm and HPLC traces were displayed at $210.3 \mathrm{~nm}$. Experiments were performed a minimum of three times. 


\section{Examination of bacterial supernatants for PAA in SCFM media}

Cultures were grown overnight on LB agar and a full loopful was inoculated into $100 \mathrm{~mL}$ of SCFM in $250 \mathrm{~mL}$ flasks. As described for LB media, supernatants were collected and extracted after 24 hours of growth. Crude extracts were dissolved in methanol to a concentration of approximately $2 \mathrm{mg} / \mathrm{ml}$ and analyzed on the same HPLC and column. The solvents selected for SCFM extracted supernatants were acetonitrile and $0.075 \%$ aqueous trifluoracetic acid. The gradient was held at $5 \%$ acetonitrile in $0.075 \%$ aqueous trifluoracetic acid for 10 minutes, increased to $20 \%$ acetonitrile over 10 minutes following a linear gradient and held there for 20 minutes. This was decreased to $5 \%$ acetonitrile for 10 minutes using a linear gradient and held for 10 minutes. The total run time was 60 minutes with a flow rate of $1 \mathrm{~mL} /$ minute. The eluent was monitored continuously at 210-600 $\mathrm{nm}$ and HPLC traces were displayed at $210.3 \mathrm{~nm}$. The experiments were performed in triplicate.

\section{Examination of fungal supernatants for PAA}

A fungal plug of A. fumigatus UAMH 2978 was inoculated into $100 \mathrm{ml}$ of potato dextrose broth (PDB or SCFM) and grown at $37^{\circ} \mathrm{C}$ with aeration at $150 \mathrm{rpm}$ for 5 days. Cultures were filtered through cheesecloth and the supernatant was acidified to a $\mathrm{pH}$ of $\leq 2$. Extractions and analysis were performed as described for bacterial supernatants in SCFM and the experiments were performed in duplicate. 
Fungal and bacterial agar plate interaction assay

A. fumigatus UAMH 2978 was grown for 5 days at $37^{\circ} \mathrm{C}$ in a conidial flask of Potato Dextrose Agar (PDA). Spores were collected in isotonic saline $+0.005 \%$ Tween 20 . Fungal spores were counted using a haemocytometer and diluted to a concentration of $10^{5}$ spores $/ \mathrm{ml}$. Bacterial cultures were standardized to $10^{7} \mathrm{cfu} / \mathrm{ml}$ using a McFarland standard and dilutions were plated on LB agar for confirmation. Based on the experiments performed using Candida albicans (Morales et al. 2013), $10 \mu 1$ of $A$. fumigatus spore suspension was streaked onto the agar plate (PDA or SCFM) and $10 \mu 1$ of bacterial culture was spotted $0.75 \mathrm{~cm}$ from the end of the streak ( 3 replicates per plate). Plates were incubated right side up at $37^{\circ} \mathrm{C}$ overnight and then incubated upside down. Plates were observed every 24 hours and photos were taken using a Leica DFC425 digital camera attached to a Leica M80 stereomicroscope integrated using Leica Application Suites version 3.7. Photos of SCFM plates were taken against a black background for contrast. Experiments were performed in triplicate.

\section{Results and Discussion}

Computational analysis of the phenylalanine catabolic pathway in CF pathogens

We examined the phenylalanine and PAA aerobic catabolism of P. aeruginosa PAO1, $B$. cenocepacia J2315 and A. fumigatus Af293 using the KEGG database. Although $A$. fumigatus has not been described as a PAA producer, another fungal species belonging to the Aspergillaceae, Penicillium chrysogenum, was able to produce and release PAA (Veiga et al. 2012). According to KEGG, each of the selected CF pathogen genomes was missing genes encoding the enzymes responsible for various steps of the pathway. The 
metabolism of phenylalanine through PAA and subsequent degradation of PAA to acetylCoA and succinyl-CoA of two model organisms, P. putida and E. coli, has been thoroughly studied (Ferrandez et al. 1998; Grishin et al. 2011; Ismail et al. 2003; Jimenez et al. 2002; Nogales et al. 2007; Teufel et al. 2010; Teufel et al. 2012). Therefore, for the steps with no gene product assigned, we used the genes from these model organisms to perform bidirectional BLASTP searches against the genomes of the selected CF pathogens.

Bidirectional BLASTP searches of P. putida KT2440 and E. coli K-12 MG1655 against each other were performed to confirm the completeness of the pathways in these microorganisms (Supplemental File S1). By performing this analysis, we were able to identify PP_3280 of P. putida KT2440 as a homolog to the E. coli K-12 MG1655 paaJ (b1397) gene (Supplemental File S1 and Supplemental Fig. S2, steps 15 and 18). PP_3280 was not indicated as a putative paaJ gene in KEGG, but is annotated as a phenylalanine degradation gene, phaD, from another study (Jimenez et al. 2002). We then used the amino acid sequences of the genes from these strains in bidirectional BLASTP searches against the genomes of the selected CF pathogens (Supplemental File S2).

B. cenocepacia $\mathrm{J} 2315$. The KEGG organism-specific pathway map of $B$. cenocepacia J2315 contains genes encoding enzymes that theoretically enable degradation of phenylalanine and the production of PAA. However, according to KEGG, the $B$. cenocepacia J2315 genome lacks some genes encoding enzymes for PAA degradation, such as PaaZ, which hydrolyzes the ring of 2-oxepin-2(3H)-ylideneacetyl-CoA and 
subsequently oxidizes the aldehyde group to a carboxyl group (Fig. 1 and Supplemental Fig. S1 steps 13 and 14, respectively) and PaaJ, a 3-oxoadipyl-CoA/3-oxo-5,6dehydrosuberyl-CoA thiolase (Fig. 1 and Supplemental Fig. S1, steps 15 and 18) (Teufel et al. 2010).

Although missing from KEGG annotations, BCAL0408 is annotated as paaZ in $B$. cenocepacia J2315 (Law et al. 2008). However, our bidirectional BLASTP search identified BCAS0364 as the best hit to b1387 (paaZ) from E. coli K-12 MG1655 (Fig. 1 and Supplemental Fig. S1, steps 13 and 14). This discrepancy may be explained by the fact that PaaZ in E. coli is a bifunctional enzyme with an N-terminal aldehyde dehydrogenase domain and a C-terminal hydratase domain. In many other bacteria the gene present in the cluster of paa genes comprises only an aldehyde dehydrogenase domain and the gene containing the hydratase domain is found elsewhere in the genome (Grishin and Cygler 2015; Teufel et al. 2011). Indeed, BCAL0408 and BCAS0364 code for an aldehyde dehydrogenase and a hydratase, respectively. Thus, it is likely that BCAS0364 and BCAL0408 are both responsible for fulfilling the functions of the E. coli PaaZ.

We also identified BCAM2568 as a homolog of b1397, paaJ of E. coli K-12 MG1655 (Fig. 1 and Supplemental Fig. S1, step 15 and 18), and BCAL0407 as a homolog of PP_3280, the putatively identified paaJ gene of $P$. putida KT2440 by our bi-directional BLASTP search. This corroborates our previous annotation where BCAM2568 and BCAL0407 were both identified as candidate paaJ genes (Law et al. 2008). 


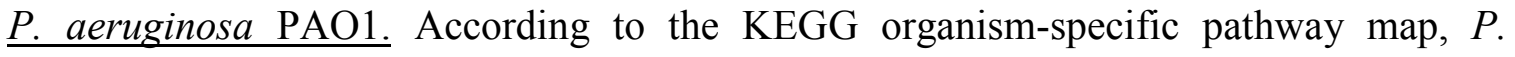
aeruginosa PAO1 lacks many coding genes for the conversion of phenylalanine to phenylacetic acid (Fig. 1). The KEGG organism-specific pathway map also shows that the PAA catabolic pathway lacks a paaK homolog for the conversion of PAA to PAACoA (Fig. 1 and Supplemental Fig. S1, step 10). Also the monoxygenase complex, paaABCDE, for the epoxidation of phenylacetyl-CoA to 2-(1,2-epoxy-1,2dihydrophenyl)acetyl-CoA (Fig. 1 and Supplemental Fig. S1, step 11) is not present, nor is paaZ (Fig. 1 and Supplemental Fig. S1, step 13 and 14) or paaJ (Fig. 1 and Supplemental Fig. S1, step 18).

The bidirectional BLASTP search for missing genes in $P$. aeruginosa PAO1 identified no alternative candidates for the conversion of phenylalanine to PAA, indicating that $P$. aeruginosa PAO1 should not be able to produce PAA from phenylalanine. For the catabolism of PAA, no paaK homolog (Fig. 1 and Supplemental Fig. S1, step 10) was identified although the gene encoding PaaI (Fig. 1 and Supplemental Fig. S1, step 9), which catalyzes the removal of CoA from PAA, is present according to KEGG. PA4889 was identified as a homolog of b1392 (PaaE) from E. coli (Fig. 1 and Supplemental Fig. $\mathrm{S} 1$, step 11), but no other genes from the monoxygenase complex were identified. PA3302 was identified in P. aeruginosa PAO1 as the best match for PaaZ from E. coli K12 MG1655 (b1387) (Fig. 1, steps 13 and 14). PA3302 is a hypothetical protein with a hotdog-fold hydratase domain, but does not contain the aldehyde dehydrogenase domain, unlike the bifunctional E. coli PaaZ. Our bidirectional BLASTP search did not find any genes with the aldehyde dehydrogenase domains similar to that of the PaaZ from $P$. putida KT2440 and E. coli K-12 MG1655. Unlike the genes for the degradation of PAA, 
which are mostly found clustered together in many bacterial genomes (Luengo et al. 2001), the putative genes in $P$. aeruginosa PAO1 do not seem to be in a cluster, therefore it is more difficult to identify a potential aldehyde dehydrogenase. Also identified by bidirectional BLASTP searches were PA0228 and PA3589, which are homologs of the $E$. coli K-12 MG1655 and P. putida KT2440 paaJ genes, b1397 and PP_3280, respectively. However, our analysis indicates that $P$. aeruginosa PAO1 does not have a complete pathway (Fig. 1) and would not be a good candidate for the production and degradation of PAA through this aerobic pathway.

A. fumigatus Af293. The KEGG organism-specific pathway map of $A$. fumigatus Af293 shows that phenylalanine can be converted by catalase-peroxidase to 2-phenylacetamide and then converted to PAA by an amidase (Fig. 1, steps 3 and 6). In A. fumigatus Af293, the aromatic-L-amino-acid decarboxylase is also present to catalyze the reaction of phenylalanine to phenylethylamine, which is then converted to phenylacetaldehyde by a monoamine oxidase (Fig. 1, steps 2 and 5). Lastly, the phenylacetaldehyde is converted to PAA by an aldehyde dehydrogenase (Fig. 1, step 8). However, for the degradation of PAA the only gene present, as identified by KEGG, is a homolog of the paaH gene responsible for the oxidation of 3-hydroxyadipyl-CoA to 3-oxyadipyl-CoA (Fig. 1 and Supplemental Fig. S1, step 17).

The bidirectional BLASTP search identified AFUA_8G02310 and AFUA_8G05520 as a homolog to the P. putida KT2440 gene and E. coli K-12 MG1655 genes, respectively, for the conversion of phenylacetaldehyde to PAA by a phenylacetaldehyde dehydrogenase (Fig. 1 and Supplemental Fig. S1, step 7), putatively providing a second method for $A$. 
fumigatus Af293 to convert phenylacetaldehyde to PAA. No PAA-CoA ligase gene (Fig. 1 and Supplemental Fig. S1, step 10), paaK, was identified and the gene for the thioesterase (Fig. 1 and Supplemental Fig. S1, step 9), paaI, was absent as well. Without the PAA-CoA, further degradation of PAA by this pathway is unlikely, as it requires CoA-thioester intermediates. Similarly to P. aeruginosa PAO1, we identified a homolog of PaaE in A. fumigatus Af293, AFUA_5G10060, but no other genes of the monoxygenase complex (Fig. 1 and Supplemental Fig. S1, step 11) were identified. Homologs to PaaG (AFUA_6G04040) and PP_3726 (AFUA_1G01890) were identified by bidirectional BLASTP search (Fig. 1 and Supplemental Fig. S1, steps 12 and 15). PaaF (AFUA_2G10920 and AFUA_3G14520) (Fig. 1 and Supplemental Fig. S1, step 16) and PaaJ (AFUA_2G11350) (Fig. 1 and Supplemental Fig. S1, steps 15 and 18) homologs were also discovered by a bidirectional BLASTP search. However, no gene homologous to PaaZ was identified for A. fumigatus Af293. The absence of a paaZ gene means the ring of 2-(1,2-epoxy-1,2-dihydrophenyl)acetyl-CoA cannot be cleaved leaving the pathway incomplete. As summarized in Fig. 1, the pathway for the degradation of PAA in A. fumigatus Af293 is incomplete. Nevertheless, the inability to degrade PAA could lead to the production of PAA by A. fumigatus Af293, which has the genes necessary to convert phenylalanine to PAA.

\section{The release of PAA by the selected $C F$ pathogens}

Based on our bioinformatics analysis, we determined that B. cenocepacia K56-2 and $A$. fumigatus Af293 may be able to produce PAA. Our bidirectional BLASTP search did not identify the genes necessary to produce PAA through this pathway in $P$. aeruginosa 
PAO1. However, we cannot rule out that other non-homologous enzymes could be involved in the production of PAA, because PAA was previously detected in $P$. aeruginosa PAO1 supernatants (Wang et al. 2013). To experimentally test the production of PAA by these CF pathogens, we tested spent media of B. cenocepacia K56-2, a clonal isolate of B. cenocepacia J2315, A. fumigatus UAMH 2978, a clinical isolate from a nonCF sputum sample, and $P$. aeruginosa PAO1 for any detectable PAA released by the organisms. Fig. 2A shows the HPLC chromatograms of bacterial extracted supernatants from LB media. The extracted LB media control had several peaks present in the chromatogram, especially from 16-22 minutes. As a positive control, $0.2 \mathrm{mM}$ of PAA was added to LB media, extracted and esterified in the same manner as the rest of the samples. PAA had a large peak at a retention time of 18.3 minutes. In the extracted supernatant of $P$. aeruginosa PAO1, the peaks were examined for a similar retention time and UV trace as PAA, however no PAA was identified. A peak with a retention time of 18.3 minutes and the same UV trace as PAA was released in B. cenocepacia K56-2 when grown in LB.

The HPLC chromatograms of extracted A. fumigatus UAMH 2978 supernatants grown in PDB are shown in Fig. 2B. The extracted PDB media control had several small peaks between 2-6 minutes and a little peak at 10 minutes and then at 18 minutes. In the positive control, $0.2 \mathrm{mM}$ of PAA was added to PDB media and then extracted. The peak of PAA was visible at 12.5 minutes. The A. fumigatus sample had a peak at 22 minutes and a peak at 24 minutes, but no peak was present with the same retention time and UV trace as PAA. 
To investigate the CF pathogens for the production of PAA in media designed to reproduce nutritional conditions of the CF sputum, the supernatants of cultures grown in synthetic cystic fibrosis sputum media (SCFM) (Palmer et al. 2007) were extracted and analyzed (Fig. 3). The extracted SCFM media control had very few peaks and they were found between 2-4 minutes. There were no peaks near the expected retention time of PAA, which was at 12.2 minutes. The extracted supernatants of $A$. fumigatus and $B$. cenocepacia have some very small peaks around the expected retention time of PAA, but they were all examined and did not have a similar UV trace to PAA. P. aeruginosa had some slight peaks when zoomed in (data not shown), but none of the peaks matched the retention time or UV trace of PAA. Under the conditions tested we did not find evidence of PAA released by the wild type strains in SCFM. We also tested B. cenocepacia K56-2 $\triangle p a a A B C D E$, a mutant that is incapable of degrading PAA, which results in the accumulation and release of PAA in common lab media (Pribytkova et al. 2014). $B$. cenocepacia $\triangle p a a A B C D E$ produced a large peak with a retention time of 12.0 minutes and a UV trace that matched PAA.

B. cenocepacia released PAA in LB, but no PAA was produced in the CF nutritional conditions of SCFM. The lack of PAA production by A. fumigatus despite the presence of a pathway that putatively should convert phenylalanine to PAA, may indicate that phenylalanine degradation is performed by the homogentisate pathway (FernandezCanon and Penalva 1995). Conversely, PAA may be produced from phenylalanine, but used as an intermediate of secondary metabolism as in other fungal strains (Veiga et al. 2012). Regarding the absence of PAA in P. aeruginosa spent medium, our experimental results were consistent with our in silico analysis, but are in disagreement with those of 
Wang et al. where they found production of PAA from $P$. aeruginosa PAO1 at high cell densities (Wang et al. 2013). Several efforts for reproducing the results of Wang et al. were attempted by collecting supernatants from $P$. aeruginosa PAO1 at different cell densities with no success in identifying PAA (data not shown). This discrepancy may be explained by different laboratory stocks of P. aeruginosa PAO1. P. aeruginosa PAO1 is commonly used as a reference strain, but genotypical and phenotypical differences have emerged amongst the sublines maintained worldwide (Klockgether et al. 2010). Also possible, is that limitations of our detection method or slight variations of the experimental conditions may have caused the discrepancy with the previous study. We consider these alternative explanations less likely as we made an effort to reproduce the same growing conditions as in Wang et al. (2013) and our method was able to detect $0.025 \mathrm{mM}$ of PAA added to LB before extraction (Supplemental Fig. S3). Wang et al. (2013) purified $\sim 20 \mu \mathrm{M}$ of PAA and predicted levels in bacterial supernatants are approximately $74-184 \mu \mathrm{M}$, therefore our method is sufficient to detect the levels of PAA present. Future work may elucidate the reasons of this disagreement.

Examining fungal-bacterial interactions of $C F$ pathogens and the role of the PAA pathway in these interactions

Analysis of soil samples revealed the co-occurrence of multiple fungi taxa with Burkholderia, and further investigation revealed that Burkholderia is able to attach to fungi and translocate using their hyphae (Stopnisek et al. 2016). It is known that Bcc, $P$. aeruginosa and A. fumigatus co-exist in the CF lung, but how they may interact with each other is unknown. The microbial interactions between $B$. cenocepacia and P. aeruginosa 
are complex. While B. cenocepacia and $P$. aeruginosa have been isolated together from the CF sputum (Jacques et al. 1998), P. aeruginosa usually kills B. cenocepacia in laboratory conditions (Tomlin et al. 2001) by synthesizing cyanide (Bernier et al. 2016). In direct contact with $A$. fumigatus, $P$. aeruginosa releases small diffusible extracellular molecules (Mowat et al. 2010), such as phenazines (Briard et al. 2015), that inhibit $A$. fumigatus. Although members of the Bcc can also inhibit A. fumigatus (Kerr 1994), it is not known which species are inhibitory, what molecules are responsible for this inhibition or the influence of the environmental conditions on this relationship. In this study, we decided to focus on these bacterial-fungal interactions and the involvement of phenylalanine and PAA metabolism of B. cenocepacia in CF nutritional conditions. Whereas PAA production inhibited protease activity, increased siderophore production, downregulated production of nematicidal protein AidA, and decreased swarming in $B$. cenocepacia (Pribytkova et al. 2014), the effect on QS-regulated antifungal activity is not known.

Bacteria were spotted on an agar plate with fungal spores streaked next to them. The interface between the fungi and bacteria was then examined for any potential morphological changes and growth inhibition. After 48 hours of growth in common lab media (PDA) there were no observable morphological or growth changes between the bacteria grown as monocultures (Fig. 4, top panels) or in the presence of the fungus (Fig. 4, lower panels). This observation is suggestive of lack of antibacterial activity by $A$. fumigatus. When B. cenocepacia and A. fumigatus were cultured on the same plate, a slight zone of inhibition was observed. This zone of inhibition was more evident when $P$. aeruginosa $\mathrm{PAO} 1$ was spotted with $A$. fumigatus. 
These results are in accordance with previous demonstrations of antifungal activity of $P$. aeruginosa and members of the Bcc against A. fumigatus (Kerr 1994). We chose E. coli OP50 as a negative control due to findings that another strain of E. coli, NCTC 10418, does not inhibit A. fumigatus (Kerr 1994). As expected, E. coli was unable to inhibit the growth of A. fumigatus.

After examining the interactions of these strains in common lab media, we used a CF sputum-mimicking medium, SCFM, to repeat the agar plate interaction method. As observed in PDA, there were no morphological or growth changes between the bacterial cultures grown alone (Fig. 5, top panels) compared to those grown in the presence of the fungus (Fig. 5, lower panels). The zone of inhibition previously seen between $B$. cenocepacia and A. fumigatus was absent in SCFM and A. fumigatus grew onto the edge of the B. cenocepacia spot. The interaction between B. cenocepacia $\triangle p a a A B C D E$, which produced a large amount of PAA in SCFM (Fig. 3), and A. fumigatus did not appear any different than that between wild type $B$. cenocepacia, which did not produce a detectable amount of PAA in SCFM, and A. fumigatus. The antifungal activity of $P$. aeruginosa was also less noticeable than on PDA, although there was a slight zone of inhibition preventing $A$. fumigatus from contacting the $P$. aeruginosa spot.

It should be noted that A. fumigatus seemed to have thinner growth on SCFM than on PDA. Despite less growth of A. fumigatus on SCFM, the antifungal activity, evidenced as a halo of inhibition at the interface between the microbes, was lessened compared to PDA. This suggests that the negative interaction between these bacteria and the fungus is lessened in CF sputum nutritional conditions, likely due a decrease of the antifungal activities by the bacteria. 
The production of pyrrolnitrin, an antifungal compound, is regulated by the CepIR QS system in members of the Bcc (Schmidt et al. 2009). Due to the inhibition of the CepIR QS system by PAA in B. cenocepacia (Pribytkova et al. 2014), we expected to see a difference in the interactions between wild type and mutant B. cenocepacia K56-2 and $A$. fumigatus. However, our results suggest that the diminished antifungal activity of $B$. cenocepacia in SCFM is independent of the PAA metabolic pathway in our experimental conditions, indicating that another non-QS-regulated antifungal compound may be responsible for the interaction.

\section{Conclusion}

We identified the presence of genes in B. cenocepacia J2315 and A. fumigatus Af293 necessary for the production of PAA through phenylalanine, but the pathway was not complete in P. aeruginosa PAO1. B. cenocepacia J2315 also has the genes for the degradation of PAA. Confirming our in silico findings, we demonstrated the release of PAA by B. cenocepacia K56-2 in LB and confirmed the absence of production of PAA by $P$. aeruginosa PAO1 under these conditions. Contrary to what the in silico analysis suggested, A. fumigatus UAMH 2978 did not release PAA in LB or PDA. We were unable to detect PAA release from any of the wild type strains tested in the CF nutritional conditions of SCFM. The antagonistic interactions between P. aeruginosa PAO1/A. fumigatus UAMH 2978 and B. cenocepacia K56-2/A. fumigatus UAMH 2978 were lessened in CF conditions when compared to common laboratory media. Using a $B$. cenocepacia K56-2 $\triangle p a a A B C D E$ mutant, deficient in the PAA catabolic pathway, we were able to demonstrate that PAA catabolic pathway dependent metabolites do not play 
a role in the antifungal activity of $B$. cenocepacia against $A$. fumigatus under the conditions tested.

\section{Acknowledgements}

This research was supported by a Natural Sciences and Engineering Research Council of Canada (NSERC) Discovery grant, awarded to S.T. Cardona. J.L. Sorensen was supported by an NSERC Discovery grant. T.J. Lightly was funded in part by the University of Manitoba, Graduate Enhancement of Tri-Council Stipends (GETS) program, and the Faculty of Science. R.R. Phung was funded by a research studentship from the Faculty of Science, University of Manitoba. Dr. Mark Belmonte kindly provided the stereomicroscope. 


\section{Reference List}

Akram, W., Anjum, T., and Ali, B. 2016. Phenylacetic Acid Is ISR Determinant Produced by Bacillus fortis IAGS162, Which Involves Extensive Re-modulation in Metabolomics of Tomato to Protect against Fusarium Wilt. Front. Plant. Sci. 7: 498.

Bakare, N., Rickerts, V., Bargon, J., and Just-Nubling, G. 2003. Prevalence of Aspergillus fumigatus and other fungal species in the sputum of adult patients with cystic fibrosis. Mycoses 46(1-2): 19-23.

Bernier, S.P., Workentine, M.L., Li, X., Magarvey, N.A., O’Toole, G.A., Surette, M.G. 2016. Cyanide Toxicity to Burkholderia cenocepacia is Modulated by Polymicrobial Communities and Environmental Factors. Front. Microbiol. 7: 725

Bhuiyan, M.S., Ellett, F., Murray, G.L., Kostoulias, X., Cerqueira, G.M., Schulze, K.E., Mahamad Maifiah, M.H., Li, J., Creek, D.J., Lieschke, G.J., and Peleg, A.Y. 2016. Acinetobacter baumannii phenylacetic acid metabolism influences infection outcome through a direct effect on neutrophil chemotaxis. Proc. Natl. Acad. Sci. U. S. A. 113(34): 9599-9604.

Briard, B., Bomme, P., Lechner, B.E., Mislin, G.L., Lair, V., Prevost, M.C., Latge, J.P., Haas, H., Beauvais, A. 2015. Pseudomonas aeruginosa manipulates redox and iron homeostasis of its microbiota partner Aspergillus fumigatus via phenazines. Sci. Rep. 5: 8220

Cheng, S.H., Gregory, R.J., Marshall, J., Paul, S., Souza, D.W., White, G.A., O'Riordan, C.R., and Smith, A.E. 1990. Defective intracellular transport and processing of CFTR is the molecular basis of most cystic fibrosis. Cell 63(4): 827-834.

Courtney, J.M., Dunbar, K.E., McDowell, A., Moore, J.E., Warke, T.J., Stevenson, M., and Elborn, J.S. 2004. Clinical outcome of Burkholderia cepacia complex infection in cystic fibrosis adults. J. Cyst Fibros 3(2): 93-98.

Courtney, J.M., Bradley, J., Mccaughan, J., O'Connor, T.M., Shortt, C., Bredin, C.P., Bradbury, I., and Elborn, J.S. 2007. Predictors of mortality in adults with cystic fibrosis. Pediatr. Pulmonol. 42(6): 525-532.

Fernandez-Canon, J.M., and Penalva, M.A. 1995. Molecular characterization of a gene encoding a homogentisate dioxygenase from Aspergillus nidulans and identification of its human and plant homologues. Journal of Biological Chemistry 270(36): 21199-21205.

Ferrandez, A., Prieto, M.A., Garcia, J.L., and Diaz, E. 1997. Molecular characterization of PadA, a phenylacetaldehyde dehydrogenase from Escherichia coli. FEBS Lett. 406(1-2): 23-27.

Ferrandez, A., Minambres, B., Garcia, B., Olivera, E.R., Luengo, J.M., Garcia, J.L., and Diaz, E. 1998. Catabolism of phenylacetic acid in Escherichia coli. Characterization of a new aerobic hybrid pathway. J. Biol. Chem. 273(40): 25974-25986.

Grishin, A.M., and Cygler, M. 2015. Structural Organization of Enzymes of the Phenylacetate Catabolic Hybrid Pathway. Biology (Basel) 4(2): 424-442. 
Grishin, A.M., Ajamian, E., Tao, L., Zhang, L., Menard, R., and Cygler, M. 2011. Structural and functional studies of the Escherichia coli phenylacetyl-CoA monooxygenase complex. J. Biol. Chem. 286(12): 10735-10743.

Hauser, A.R., Jain, M., Bar-Meir, M., and McColley, S.A. 2011. Clinical significance of microbial infection and adaptation in cystic fibrosis. Clin. Microbiol. Rev. 24(1): 29-70.

Ismail, W., El-Said Mohamed, M., Wanner, B.L., Datsenko, K.A., Eisenreich, W., Rohdich, F., Bacher, A., and Fuchs, G. 2003. Functional genomics by NMR spectroscopy. Phenylacetate catabolism in Escherichia coli. Eur. J. Biochem. 270(14): 3047-3054.

Jacques, I., Derelle, M., Weber, M., Vidailhet, M. 1998. Pulmonary evolution of cystic fibrosis colonized by Pseudomonas aeruginosa and/or Burkholderia cepacia. Eur. J. Pediatr. 157(5):427431

Jimenez, J.I., Minambres, B., Garcia, J.L., and Diaz, E. 2002. Genomic analysis of the aromatic catabolic pathways from Pseudomonas putida KT2440. Environ. Microbiol. 4(12): 824-841.

Kanehisa, M., and Goto, S. 2000. KEGG: kyoto encyclopedia of genes and genomes. Nucleic Acids Res. 28(1): 27-30.

Kanehisa, M., Sato, Y., Kawashima, M., Furumichi, M., and Tanabe, M. 2016. KEGG as a reference resource for gene and protein annotation. Nucleic Acids Res. 44(D1): D457-62.

Kerr, J. 1994. Inhibition of fungal growth by Pseudomonas aeruginosa and Pseudomonas cepacia isolated from patients with cystic fibrosis. J. Infect. 28(3): 305-310.

Klockgether, J., Munder, A., Neugebauer, J., Davenport, C.F., Stanke, F., Larbig, K.D., Heeb, S., Schock, U., Pohl, T.M., Wiehlmann, L., and Tummler, B. 2010. Genome diversity of Pseudomonas aeruginosa PAO1 laboratory strains. J. Bacteriol. 192(4): 1113-1121.

Law, R.J., Hamlin, J.N.R., Sivro, A., McCorrister, S.J., Cardama, G.A., and Cardona, S.T. 2008. A functional phenylacetic acid catabolic pathway is required for full pathogenicity of

Burkholderia cenocepacia in the Caenorhabditis elegans host model. J. Bacteriol. 190(21): 72097218 .

Lipuma, J.J. 2010. The changing microbial epidemiology in cystic fibrosis. Clin. Microbiol. Rev. 23(2): 299-323.

Luengo, J.M., Garcia, J.L., and Olivera, E.R. 2001. The phenylacetyl-CoA catabolon: a complex catabolic unit with broad biotechnological applications. Mol. Microbiol. 39(6): 1434-1442.

Mahenthiralingam, E., Urban, T.A., and Goldberg, J.B. 2005. The multifarious, multireplicon Burkholderia cepacia complex. Nat. Rev. Microbiol. 3(2): 144-156.

Mahenthiralingam, E., Coenye, T., Chung, J.W., Speert, D.P., Govan, J.R., Taylor, P., and Vandamme, P. 2000. Diagnostically and experimentally useful panel of strains from the Burkholderia cepacia complex. J. Clin. Microbiol. 38(2): 910-913. 
Martinez-Blanco, H., Reglero, A., Rodriguez-Aparicio, L.B., and Luengo, J.M. 1990. Purification and biochemical characterization of phenylacetyl-CoA ligase from Pseudomonas putida. A specific enzyme for the catabolism of phenylacetic acid. J. Biol. Chem. 265(12): 7084-7090.

Matsui, H., Grubb, B.R., Tarran, R., Randell, S.H., Gatzy, J.T., Davis, C.W., and Boucher, R.C. 1998. Evidence for periciliary liquid layer depletion, not abnormal ion composition, in the pathogenesis of cystic fibrosis airways disease. Cell 95(7): 1005-1015.

Morales, D.K., Grahl, N., Okegbe, C., Dietrich, L.E., Jacobs, N.J., and Hogan, D.A. 2013. Control of Candida albicans metabolism and biofilm formation by Pseudomonas aeruginosa phenazines. MBio 4(1): e00526-12.

Mortensen, K.L., Jensen, R.H., Johansen, H.K., Skov, M., Pressler, T., Howard, S.J., Leatherbarrow, H., Mellado, E., and Arendrup, M.C. 2011. Aspergillus species and other molds in respiratory samples from patients with cystic fibrosis: a laboratory-based study with focus on Aspergillus fumigatus azole resistance. J. Clin. Microbiol. 49(6): 2243-2251.

Mowat, E., Rajendran, R., Williams, C., McCulloch, E., Jones, B., Lang, S., and Ramage, G. 2010. Pseudomonas aeruginosa and their small diffusible extracellular molecules inhibit Aspergillus fumigatus biofilm formation. FEMS Microbiol. Lett. 313(2): 96-102.

Musthafa, K.S., Sivamaruthi, B.S., Pandian, S.K., and Ravi, A.V. 2012. Quorum sensing inhibition in Pseudomonas aeruginosa PAO1 by antagonistic compound phenylacetic acid. Curr. Microbiol. 65(5): 475-480.

Nogales, J., Macchi, R., Franchi, F., Barzaghi, D., Fernandez, C., Garcia, J.L., Bertoni, G., and Diaz, E. 2007. Characterization of the last step of the aerobic phenylacetic acid degradation pathway. Microbiology 153(Pt 2): 357-365.

Palmer, K.L., Aye, L.M., and Whiteley, M. 2007. Nutritional cues control Pseudomonas aeruginosa multi-cellular behavior in cystic fibrosis sputum. J. Bacteriol. 189(22): 8079-8087.

Paugam, A., Baixench, M.T., Demazes-Dufeu, N., Burgel, P.R., Sauter, E., Kanaan, R., Dusser, D., Dupouy-Camet, J., and Hubert, D. 2010. Characteristics and consequences of airway colonization by filamentous fungi in 201 adult patients with cystic fibrosis in France. Med. Mycol. 48 Suppl 1: S32-6.

Pribytkova, T., Lightly, T.J., Kumar, B., Bernier, S.P., Sorensen, J.L., Surette, M.G., and Cardona, S.T. 2014. The attenuated virulence of a Burkholderia cenocepacia paaABCDE mutant is due to inhibition of quorum sensing by release of phenylacetic acid. Mol. Microbiol. 94(3): 522-536.

Riordan, J.R., Rommens, J.M., Kerem, B., Alon, N., Rozmahel, R., Grzelczak, Z., Zielenski, J., Lok, S., Plavsic, N., and Chou, J.L. 1989. Identification of the cystic fibrosis gene: cloning and characterization of complementary DNA. Science 245(4922): 1066-1073.

Schmidt, S., Blom, J.F., Pernthaler, J., Berg, G., Baldwin, A., Mahenthiralingam, E., Eberl, L. 2009. Production of the antifungal compound pyrrolnitrin is quorum sensing-regulated in members of the Burkholderia cepacia complex. Environ. Microbiol. 11(6): 1422-1437. 
Shiddiqui, I.A., and Shaukat, S.S. 2004. Phenylacetic acid-producing Rhizoctonia solani represses the biosynthesis of nematicidal compounds in vitro and influences biocontrol of Meloidogyne incognita in tomato by Pseudomonas fluorescens strain CHA0 and its GM derivatives. J. Appl. Microbiol. 98(1): 43-55

Sibley, C.D., and Surette, M.G. 2011. The polymicrobial nature of airway infections in cystic fibrosis: Cangene Gold Medal Lecture. Can. J. Microbiol. 57(2): 69-77.

Sibley, C.D., Rabin, H., and Surette, M.G. 2006. Cystic fibrosis: a polymicrobial infectious disease. Future Microbiol. 1(1): 53-61.

Smith, K., Rajendran, R., Kerr, S., Lappin, D.F., Mackay, W.G., Williams, C., and Ramage, G. 2015. Aspergillus fumigatus enhances elastase production in Pseudomonas aeruginosa cocultures. Medical Mycology 53(7): 645-655.

Steinebach, V., Benen, J.A., Bader, R., Postma, P.W., De Vries, S., and Duine, J.A. 1996. Cloning of the maoA gene that encodes aromatic amine oxidase of Escherichia coli W3350 and characterization of the overexpressed enzyme. Eur. J. Biochem. 237(3): 584-591.

Stopnisek, N., Zuhlke, D., Carlier, A., Barberan, A., Fierer, N., Becher, D., Riedel, K., Eberl, L., and Weisskopf, L. 2016. Molecular mechanisms underlying the close association between soil Burkholderia and fungi. ISME J. 10(1): 253-264.

Teufel, R., Friedrich, T., and Fuchs, G. 2012. An oxygenase that forms and deoxygenates toxic epoxide. Nature 483(7389): 359-362.

Teufel, R., Gantert, C., Voss, M., Eisenreich, W., Haehnel, W., and Fuchs, G. 2011. Studies on the mechanism of ring hydrolysis in phenylacetate degradation: a metabolic branching point. J. Biol. Chem. 286(13): 11021-11034.

Teufel, R., Mascaraque, V., Ismail, W., Voss, M., Perera, J., Eisenreich, W., Haehnel, W., and Fuchs, G. 2010. Bacterial phenylalanine and phenylacetate catabolic pathway revealed. Proc. Natl. Acad. Sci. U. S. A. 107(32): 14390-14395.

Tomlin, K.L., Coll, O.P., Ceri, H. 2001. Interspecies biofilms of Pseudomonas aeruginosa and Burkholderia cepacia. Can. J. Microbiol. 47(10): 949-954.

Veiga, T., Solis-Escalante, D., Romagnoli, G., ten Pierick, A., Hanemaaijer, M., Deshmukh, A.T., Wahl, A., Pronk, J.T., and Daran, J.M. 2012. Resolving phenylalanine metabolism sheds light on natural synthesis of penicillin G in Penicillium chrysogenum. Eukaryot. Cell. 11(2): 238-249.

Vitovski, S. 1993. Phenylacetate-coenzyme A ligase is induced during growth on phenylacetic acid in different bacteria of several genera. FEMS Microbiol Lett. 108: 1-5.

Wang, J., Dong, Y., Zhou, T., Liu, X., Deng, Y., Wang, C., Lee, J., and Zhang, L.H. 2013. Pseudomonas aeruginosa cytotoxicity is attenuated at high cell density and associated with the accumulation of phenylacetic acid. PLoS One 8(3): e60187. 
Table 1. Bacterial and fungal strains used in this study.

\begin{tabular}{|c|c|c|}
\hline Strains & Features & Reference or Source \\
\hline \multicolumn{3}{|l|}{ Burkholderia cenocepacia } \\
\hline K56-2 (LMG18863) & $\begin{array}{l}\text { WT strain, ET } 12 \text { clone } \\
\text { related to a cystic fibrosis } \\
\text { clinical isolate, } \mathrm{J} 2315\end{array}$ & $\begin{array}{l}\text { (Mahenthiralingam et al. } \\
\text { 2000) }\end{array}$ \\
\hline$\triangle p a a A B C D E$ & $\begin{array}{l}\text { Deletion of paaABCDE } \\
\text { operon in K56-2 }\end{array}$ & (Pribytkova et al. 2014) \\
\hline \multicolumn{3}{|l|}{ Escherichia coli } \\
\hline OP50 & Uracil auxotroph & $\begin{array}{l}\text { Caenorhabditis Genetic } \\
\text { Center (CGC, University of } \\
\text { Minnesota, Minneapolis, } \\
\text { USA) }\end{array}$ \\
\hline \multicolumn{3}{|l|}{ Pseudomonas aeruginosa } \\
\hline PAO1 & Clinical isolate & $\begin{array}{l}\text { Courtesy of Dr. Teresa } \\
\text { deKievit, University of } \\
\text { Manitoba, Canada }\end{array}$ \\
\hline \multicolumn{3}{|l|}{ Aspergillus fumigatus } \\
\hline UAMH 2978 & $\begin{array}{l}\text { Sputum ex adult, } \\
\text { thermotolerant }\end{array}$ & $\begin{array}{l}\text { University of Alberta } \\
\text { Microfungus Collection and } \\
\text { Herbarium (UAMH, } \\
\text { Alberta, Canada) }\end{array}$ \\
\hline
\end{tabular}




\section{Figure legends}

Fig. 1. Putative phenylalanine (Phe) catabolic pathways of selected CF pathogens. Using the KEGG database and bidirectional NCBI BLASTP searches, the phenylalanine catabolic pathway was determined for three CF pathogens. Numbered arrows correspond to those shown in Supplemental Fig. S1 and represent the steps in the pathway, which are catalyzed by enzymes encoded by one or more genes. Green arrows indicate enzymatic steps where the presence of the gene(s) has been identified by KEGG with the locus tags in green boxes. Red arrows are for steps with gene(s) absent as indicated by KEGG and bidirectional BLASTP search (this study). Blue arrows represent steps where the gene(s) were putatively identified by bidirectional BLASTP search in this study (locus tags in blue boxes). An asterix marks steps where although a gene is present, the step cannot be completed due to missing genes.

Fig. 2. HPLC analysis of culture extracts grown in common lab media. A) Esterified ethyl acetate extracts from LB media were compared. The top trace (PAA) is the extract of $0.2 \mathrm{mM}$ PAA in LB with the UV trace of PAA (inset) and the bottom trace (LB) is the extract of the media control. B. cenocepacia K56-2 (Bce) had a peak at 18.3 minutes with a UV trace (inset) that resembles that of PAA. P. aeruginosa PAO1 (Pae) was also tested and no PAA was detected. B) Ethyl acetate extracts from potato dextrose broth (PDB) were examined. The top trace (PAA) was the extract of $0.2 \mathrm{mM}$ PAA in PDB and the bottom trace was the extract of the media (PDB). The extract of A. fumigatus UAMH2978 (Afu) had no peaks with a retention time similar to PAA. Chromatograms are from one replicate, but are representative of at least two biological replicates.

Fig. 3. HPLC analysis of culture extracts grown in SCFM. The HPLC traces at 210.3 $\mathrm{nm}$ were examined for the presence of PAA. The top trace (PAA) is the ethyl acetate extract of $0.2 \mathrm{mM}$ PAA in SCFM and the bottom trace is the ethyl acetate extract from the media alone. The peak of PAA had a retention time of 12.2 minutes and the inset is the UV spectra recorded for this signal. B. cenocepacia K56-2 $\triangle p a a A B C D E$ (Bce $\triangle \mathrm{paa}$ ) is the only trace where a similar peak was found, with a retention time of 12.0 minutes and an identical UV spectra. All experiments were performed at least two times. 
Fig. 4. Fungal interaction assay on potato dextrose agar. Bce: B. cenocepacia K56-2; Eco: E. coli OP50; Pae: P. aeruginosa PAO1; Afu: A. fumigatus UAMH 2978. The top row is the colony morphology of monocultures after $48 \mathrm{~h}$. The bottom row is the colony morphology of each bacterial strain when grown next to A. fumigatus UAMH 2978 for 48 h. B. cenocepacia K56-2 slightly inhibited the growth of $A$. fumigatus UAMH 2978. E. coli OP50 was unable to inhibit $A$. fumigatus and $P$. aeruginosa PAO1 had a clear zone of inhibition. Images were obtained using a stereomicroscope $(2 \mathrm{x})$ and the experiment was repeated three times.

Fig. 5. Fungal interaction assay on SCFM. Bce: $B$. cenocepacia K56-2; Bce $\Delta$ paa: $B$. cenocepacia K56-2 $\triangle p a a A B C D E$; Eco: E. coli OP50; Pae: P. aeruginosa PAO1; Afu: A. fumigatus UAMH 2978. The top row is the colony morphology of monocultures after 48 $\mathrm{h}$. The bottom row is the colony morphology of each bacterial strain when grown next to A. fumigatus UAMH 2978 for 48 h. Both B. cenocepacia K56-2 wild type and mutant $\triangle p a a A B C D E$ had similar interactions with $A$. fumigatus UAMH 2978 as they were unable to inhibit the growth of $A$. fumigatus UAMH 2978 on SCFM. E. coli OP50 was unable to inhibit $A$. fumigatus UAMH 2978. P. aeruginosa PAO1 inhibited $A$. fumigatus UAMH 2978 slightly. Experiments were performed in triplicate and images were obtained using a stereomicroscope $(2 \mathrm{x})$. 

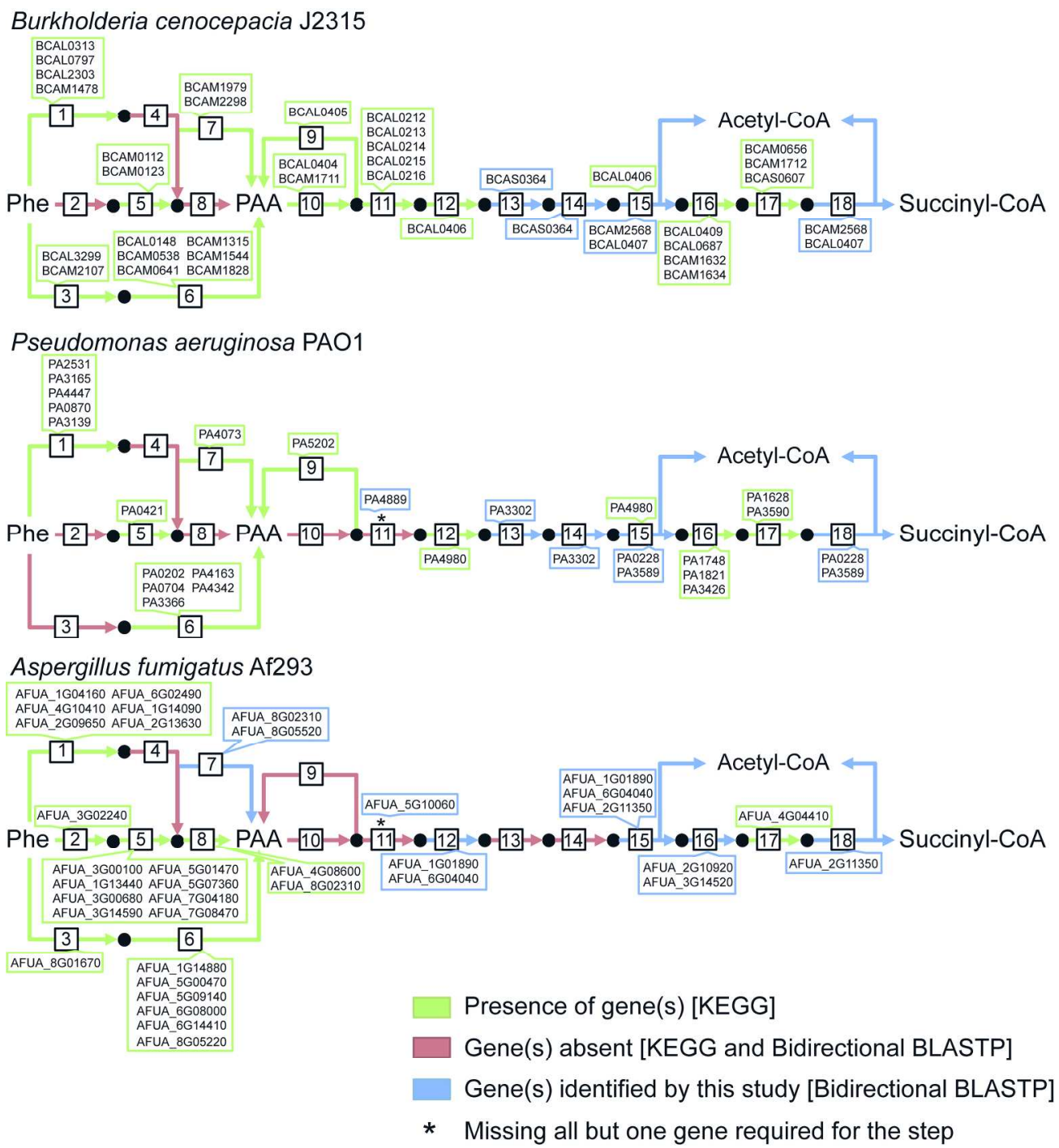

Fig. 1. Putative phenylalanine (Phe) catabolic pathways of selected CF pathogens. Using the KEGG database and bidirectional NCBI BLASTP searches, the phenylalanine catabolic pathway was determined for three CF pathogens. Numbered arrows correspond to those shown in Supplemental Fig. S1 and represent the steps in the pathway, which are catalyzed by enzymes encoded by one or more genes. Green arrows indicate enzymatic steps where the presence of the gene(s) has been identified by KEGG with the locus tags in green boxes. Red arrows are for steps with gene(s) absent as indicated by KEGG and bidirectional BLASTP search (this study). Blue arrows represent steps where the gene(s) were putatively identified by bidirectional BLASTP search in this study (locus tags in blue boxes). An asterix marks steps where although a gene is present, the step cannot be completed due to missing genes.

Fig. 1

$199 \times 217 \mathrm{~mm}(300 \times 300 \mathrm{DPI})$ 


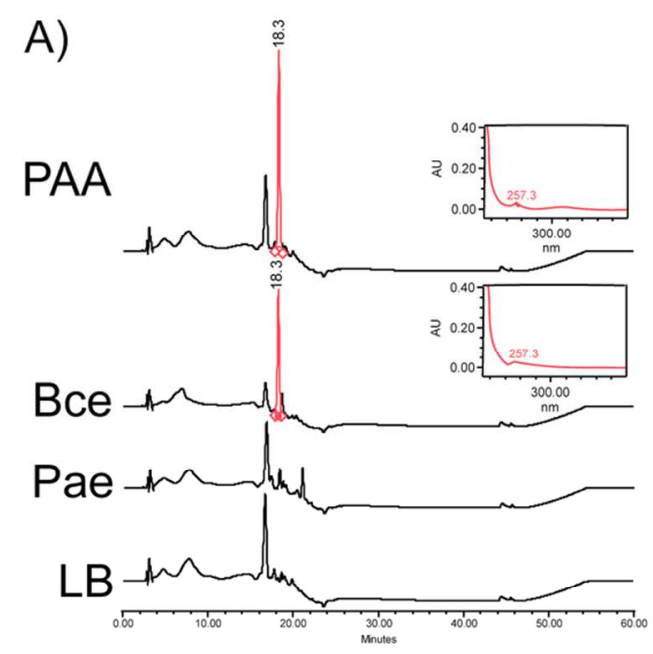

B)

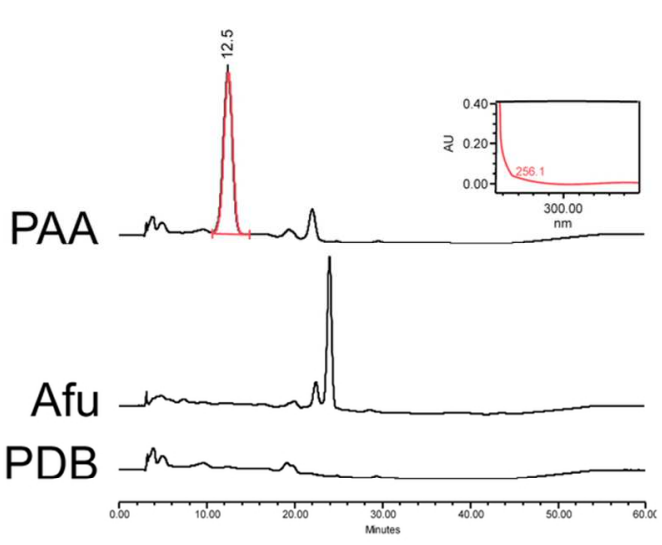

Fig. 2. HPLC analysis of culture extracts grown in common lab media. A) Esterified ethyl acetate extracts from LB media were compared. The top trace (PAA) is the extract of 0.2 mM PAA in LB with the UV trace of PAA (inset) and the bottom trace (LB) is the extract of the media control. B. cenocepacia K56-2 (Bce) had a peak at 18.3 minutes with a UV trace (inset) that resembles that of PAA. $P$. aeruginosa PAO1 (Pae) was also tested and no PAA was detected. B) Ethyl acetate extracts from potato dextrose broth (PDB) were examined. The top trace (PAA) was the extract of $0.2 \mathrm{mM}$ PAA in PDB and the bottom trace was the extract of the media (PDB). The extract of $A$. fumigatus UAMH2978 (Afu) had no peaks with a retention time similar to PAA. Chromatograms are from one replicate, but are representative of at least two biological replicates.

Fig. 2

$95 \times 51 \mathrm{~mm}(300 \times 300$ DPI $)$ 


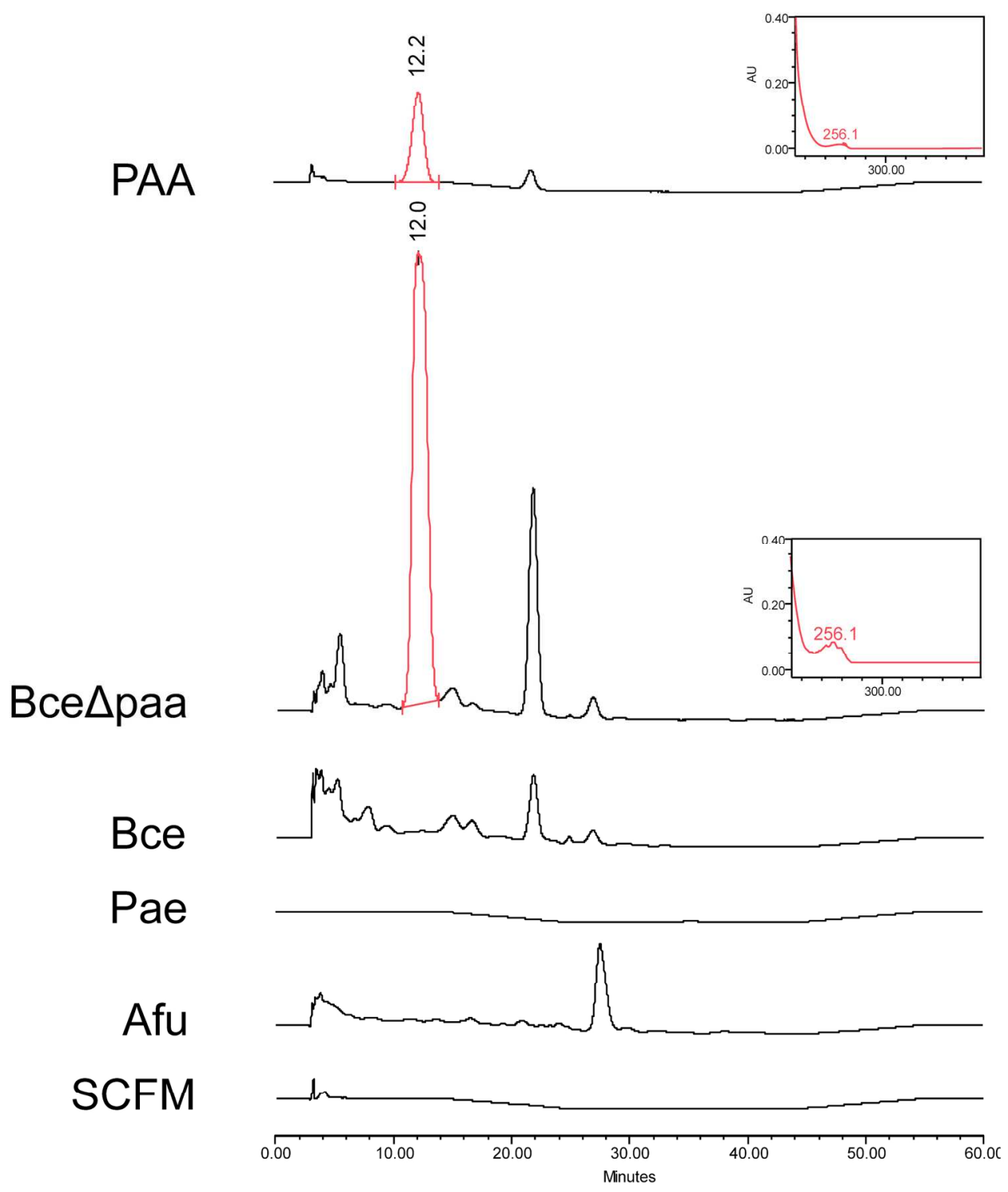

Fig. 3. HPLC analysis of culture extracts grown in SCFM. The HPLC traces at $210.3 \mathrm{~nm}$ were examined for the presence of PAA. The top trace (PAA) is the ethyl acetate extract of $0.2 \mathrm{mM}$ PAA in SCFM and the bottom trace is the ethyl acetate extract from the media alone. The peak of PAA had a retention time of 12.2 minutes and the inset is the UV spectra recorded for this signal. B. cenocepacia K56-2 $\triangle$ paaABCDE (Bce $\triangle \mathrm{paa}$ ) is the only trace where a similar peak was found, with a retention time of 12.0 minutes and an identical UV spectra. All experiments were performed at least two times.

Fig. 3

$172 \times 200 \mathrm{~mm}(300 \times 300 \mathrm{DPI})$ 

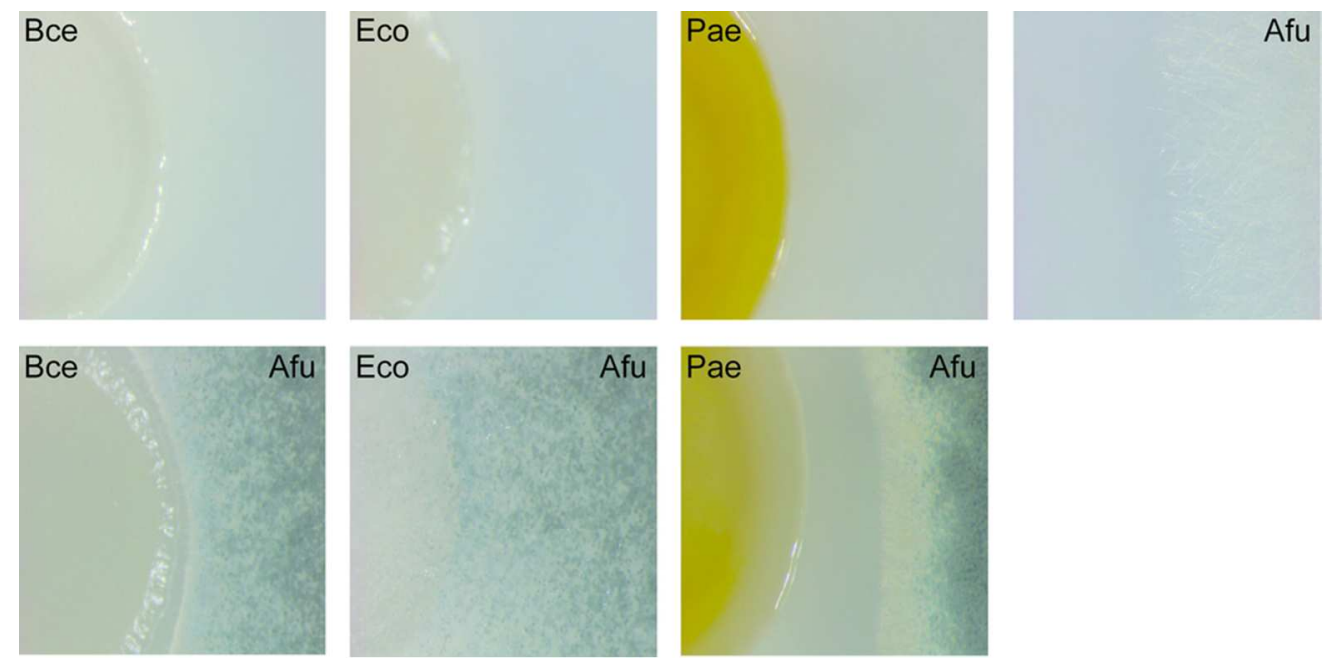

Fig. 4. Fungal interaction assay on potato dextrose agar. Bce: $B$. cenocepacia $\mathrm{K} 56-2$; Eco: $E$. coli OP50; Pae: P. aeruginosa PAO1; Afu: A. fumigatus UAMH 2978. The top row is the colony morphology of monocultures after $48 \mathrm{~h}$. The bottom row is the colony morphology of each bacterial strain when grown next to $A$. fumigatus UAMH 2978 for 48 h. B. cenocepacia K56-2 slightly inhibited the growth of $A$. fumigatus UAMH 2978. E. coli OP50 was unable to inhibit $A$. fumigatus and $P$. aeruginosa PAO1 had a clear zone of inhibition. Images were obtained using a stereomicroscope $(2 x)$ and the experiment was repeated three times.

Fig. 4

$86 \times 43 \mathrm{~mm}(300 \times 300 \mathrm{DPI})$ 

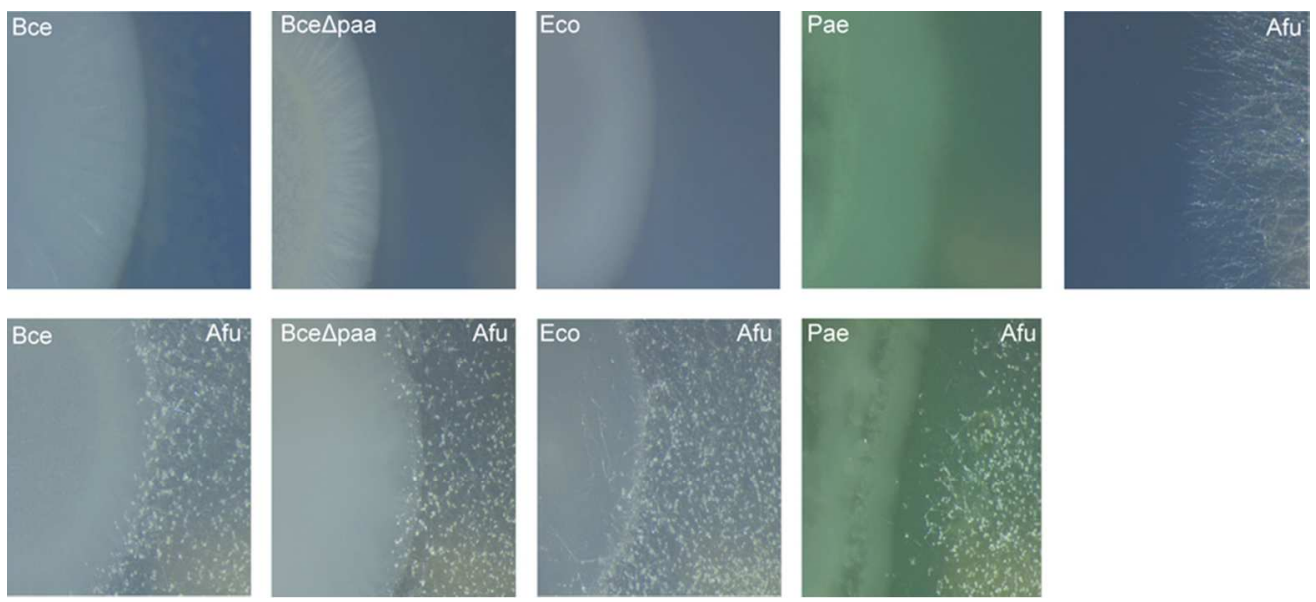

Fig. 5. Fungal interaction assay on SCFM. Bce: B. cenocepacia K56-2; Bce $\Delta$ paa: B. cenocepacia K56-2 $\triangle$ paaABCDE; Eco: E. coli OP50; Pae: P. aeruginosa PAO1; Afu: A. fumigatus UAMH 2978. The top row is the colony morphology of monocultures after $48 \mathrm{~h}$. The bottom row is the colony morphology of each bacterial strain when grown next to $A$. fumigatus UAMH 2978 for 48 h. Both $B$. cenocepacia K56-2 wild type and mutant $\triangle$ paaABCDE had similar interactions with $A$. fumigatus UAMH 2978 as they were unable to inhibit the growth of $A$. fumigatus UAMH 2978 on SCFM. E. coli OP50 was unable to inhibit the growth of $A$. fumigatus UAMH 2978. $P$. aeruginosa PAO1 inhibited $A$. fumigatus UAMH 2978 slightly. Experiments were performed in triplicate and images were obtained using a stereomicroscope $(2 x)$.

Fig. 5

$78 \times 35 \mathrm{~mm}(300 \times 300$ DPI $)$ 
Supplemental File 1: Bi-directional BLASTP from P. putida KT2440 to E. coli K-12 MG:

Overview:

\section{Bidirectional BLASTP (examplı}

BLASTP against
P. putida
E. coli genome
gene A

BLASTP against

P. putida E. coligenome gene B

Each tab contains the results from a BLASTP search

Tab \#

Tab 1

Tab 2

Tab 3

Tab 4

Tab 5

Tab 6

Tab 7

\section{Tab Title}

About File

$\mathrm{KT} 2440$ to $\mathrm{K} 12$

K12 back to KT2440

Bidirectional KT2440 to K12

K12 to KT2440

KT2440 back to K12

Bidirectional K12 to KT2440

\section{Contents}

Overview of Supplemental File 1

Contains the results from a BLASTP search using $P$. put E. coli K-12 MG1655 genes from the BLASTP search ag: Summary of the locus tag of the initial query gene (P. $p$ Contains the results from a BLASTP search using $E$. coli P. putida K-12 MG1655 genes from the BLASTP search Summary of the locus tag of the initial query gene ( $E . C$ 
e P. putida to E. coli)
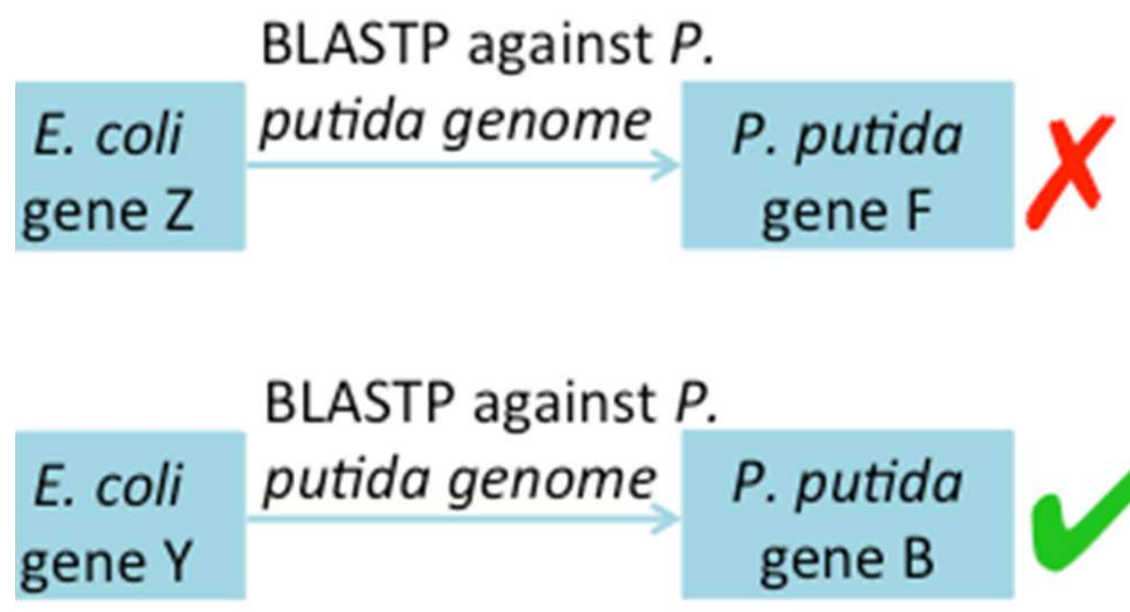

ida KT2440 genes as queries against the $E$. coli $\mathrm{K}-12 \mathrm{MG} 1655$ genome ainst the $E$. coli K-12 MG1655 genome were used in a BLASTP search against the P. putida KT244 Iutida $\mathrm{KT} 2440)$, the resulting hit from the E. coli $\mathrm{K}-12 \mathrm{MG} 1655$ genome and the resulting hit of $\mathrm{tl}$ K12-MG1655 genes as queries against the $P$. putida KT2440 genome against the $P$. putida $\mathrm{KT} 2440$ genome were used in a BLASTP search against the $E$. coli K-12 MG oli K-12 MG1655), the resulting hit from the P. putida KT2440 genome and the resulting hit of $\mathrm{tl}$ 
10 genome

רe $E$. coli K-12 MG1655 gene against the $P$. putida KT2440 genome 1655 genome

1e $P$. putida KT2440 gene against the $E$. coli K-12 MG1655 genome 
Pseudomonas putida KT2440 protein sequences from pseudomonas.com BLAST against $E$. coli M

Query

$P$. putida gene name

hisC

tyrB-1

tyrB-2

phaE

phal

phaH

paaB

paaA

phaB

phat

fadB1x

PP_3283

PP_3726

\section{Result}

\section{Locus Tag Description}

PP_3668

PP_2932

MULTISPECIES: catalase peroxidase [Proteobacteria]

No Significant Similarity Found

MULTISPECIES: ABC transporter ATPase

PP_2552 [Proteobacteria]

MULTISPECIES: N-methyl-L-tryptophan oxidase

PP_4983 [Proteobacteria]

MULTISPECIES: NAD-dependent phenylacetaldehyde

PP_3463 dehydrogenase [Escherichia]

PP_0967 No Significant Similarity Found

MULTISPECIES: aromatic amino acid aminotransferase

PP_1972 [Proteobacteria]

MULTISPECIES: aromatic amino acid aminotransferase

PP_3590 [Proteobacteria]

MULTISPECIES: phenylacetate--CoA ligase

PP_3279 [Enterobacteriaceae]

MULTISPECIES: 1,2-phenylacetyl-CoA epoxidase

PP_3274 subunit E [Enterobacteriaceae]

MULTISPECIES: 1,2-phenylacetyl-CoA epoxidase

PP_3275 [Enterobacteriaceae]

MULTISPECIES: 1,2-phenylacetyl-CoA epoxidase

PP_3276 subunit C [Enterobacteriaceae]

MULTISPECIES: 1,2-phenylacetyl-CoA epoxidase

PP_3277 subunit B [Enterobacteriaceae]

MULTISPECIES: 1,2-phenylacetyl-CoA epoxidase

PP_3278 subunit A [Enterobacteriaceae]*

MULTISPECIES: 2-(1,2-epoxy-1,2-dihydrophenyl)acetyl-

CoA isomerase [Enterobacteriaceae]

MULTISPECIES: 2,3-dehydroadipyl-CoA hydratase

[Enterobacteriaceae]

MULTISPECIES: phenylacetic acid degradation

PP_3270 bifunctional protein PaaZ [Escherichia]

PP_1845 No Significant Similarity Found

MULTISPECIES: 2,3-dehydroadipyl-CoA hydratase

PP_2217 [Enterobacteriaceae]

MULTISPECIES: 2,3-dehydroadipyl-CoA hydratase

PP_3284 [Enterobacteriaceae] 


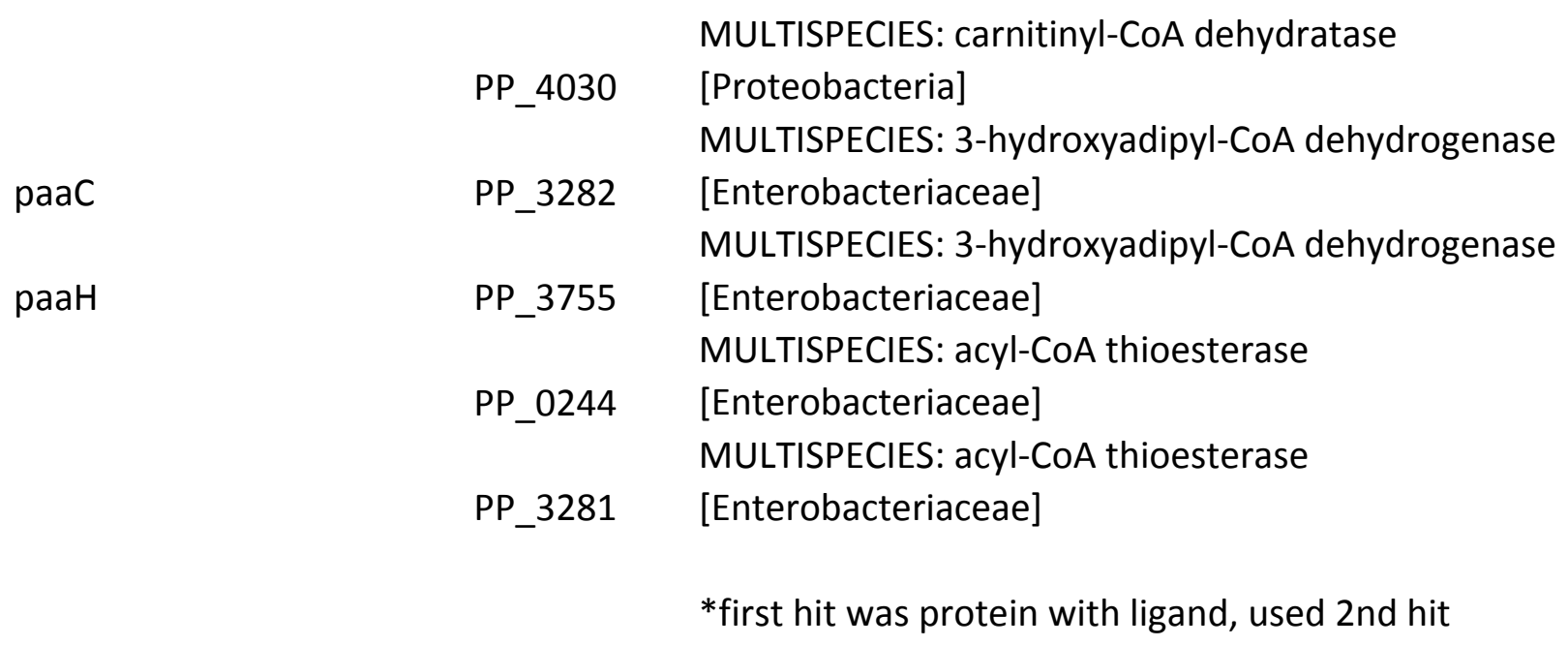




\section{G1655 (taxid:511145)}

\begin{tabular}{|c|c|c|c|c|c|c|}
\hline Max Score & Total Score & Query cover & E value & Ident & Accession & E. coli Locus \\
\hline 885 & 885 & $99 \%$ & 0 & $63 \%$ & WP_001295695.1 & b3942 \\
\hline 26.2 & 26.2 & $9 \%$ & 5.4 & $37 \%$ & WP_001300558.1 & b1756 \\
\hline 30.4 & 30.4 & $4 \%$ & 0.45 & $52 \%$ & WP_000872833.1 & b1059 \\
\hline 419 & 419 & $98 \%$ & $8.00 \mathrm{E}-144$ & $43 \%$ & WP_000138615.1 & b1385 \\
\hline 418 & 418 & $99 \%$ & $3.00 \mathrm{E}-146$ & $51 \%$ & 6P_000486985.1 & b4054 \\
\hline 590 & 590 & $99 \%$ & $0.00 E+00$ & $71 \%$ & WP_000486985.1 & b4054 \\
\hline 614 & 614 & $96 \%$ & 0 & $68 \%$ & WP_000632280.1 & b1398 \\
\hline 350 & 350 & $99 \%$ & $1.00 \mathrm{E}-120$ & $49 \%$ & WP_000206388.1 & b1392 \\
\hline 176 & 176 & $88 \%$ & $7.00 \mathrm{E}-58$ & $54 \%$ & WP_001189201.1 & b1391 \\
\hline 258 & 258 & $98 \%$ & 2.00E-91 & $54 \%$ & WP_001072837.1 & b1390 \\
\hline 125 & 125 & $96 \%$ & $5.00 E-40$ & $61 \%$ & WP_000073393.1 & b1389 \\
\hline 456 & 456 & $94 \%$ & $1.00 \mathrm{E}-163$ & $68 \%$ & WP_000191077.1 & b1388 \\
\hline 308 & 308 & $99 \%$ & 4.00E-107 & $62 \%$ & WP_000969784.1 & b1394 \\
\hline 142 & 142 & $98 \%$ & $2.00 E-42$ & $36 \%$ & WP_001292353.1 & b1393 \\
\hline 732 & 732 & $98 \%$ & 0 & $59 \%$ & WP_001186469.1 & b1387 \\
\hline 200 & 200 & $95 \%$ & 7.00E-65 & $43 \%$ & WP_001292353.1 & b1393 \\
\hline 267 & 267 & $96 \%$ & $3.00 E-91$ & $56 \%$ & WP_001292353.1 & b1393 \\
\hline
\end{tabular}




$\begin{array}{rrrrrr}128 & 128 & 91 \% & 2.00 \mathrm{E}-36 & 34 \% \text { WP_001295419.1 } & \text { b0036 } \\ 410 & 410 & 96 \% & 2.00 \mathrm{E}-140 & 48 \% \text { WP_000973360.1 } & \mathrm{b} 1395 \\ 219 & 303 & 100 \% & 3.00 \mathrm{E}-69 & 41 \% \text { WP_000973360.1 } & \mathrm{b} 1395 \\ 61.2 & 61.2 & 91 \% & 8.00 \mathrm{E}-14 & 36 \% \text { WP_000018413.1 } & \mathrm{b} 1396 \\ 124 & 124 & 89 \% & 5.00 \mathrm{E}-38 & 47 \% \text { WP_000018413.1 } & \text { b1396 }\end{array}$




\section{GenBank Accession E. coli Gene Name}

AAC76924.1 katG

$\begin{array}{ll}\text { AAC74826.1 } & \text { ynjD } \\ \text { AAC74143.1 } & \text { solA } \\ \text { AAC74467.2 } & \text { feaB }\end{array}$

AAC77024.1 tyrB

AAC77024.1 tyrB

AAC74480.1 paak

AAC74474.1 paaE

AAC74473.4 paaD

AAC74472.1 paaC

AAC74471.1 раaB

AAC74470.1 paaA

AAC74476.1 paaG

AAC74475.1 paaF

AAC74469.1 paaZ

AAC74475.1 paaF

AAC74475.1 paaF 
AAC73147.2 caiD

AAC74477.1 paaH

AAC74477.1 paaH

AAC74478.1 paal

AAC74478.1 paal 
Reverse Blast of $E$. coli $\mathrm{K}-12$ gene results fror

Query

P. putida Locus

E. coli gene name $E$. coli Locus Initial Query

katG

b3942 PP_3668

PP_2932

ynjD

b1756

PP_2552

solA

b1059

PP_4983

feaB

b1385

PP_3463

PP_0967

tyrB

b4054

PP_1972

tyrB

b4054

PP_3590

paak

b1398

PP_3279

paaE

b1392

PP_3274

paaD

b1391

PP_3275

paaC

b1390

PP_3276

paaB

b1389

PP_3277

paaA

b1388

PP_3278

paaG

b1394

PP_3283

paaF

b1393

PP_3726

paaZ

b1387

PP_3270

PP_1845

paaF

b1393

PP_2217

paaF

b1393

PP_3284

b0036

PP_4030

paaH

b1395

PP_3282 


$\begin{array}{lll}\text { paaH } & \text { b1395 } & \text { PP_3755 } \\ \text { paal } & \text { b1396 } & \text { PP_0244 } \\ \text { paal } & \text { b1396 } & \text { PP_3281 }\end{array}$


n P. putida KT2440 to E. coli K12 against $P$. putida KT2440 (taxid:160488)

Result

\section{Description}

catalase peroxidase [Pseudomonas putida]
Max Score Total Score Query cover 888

888

$97 \%$

[Pseudomonas]

MULTISPECIES: aromatic amino acid aminotransferase [Pseudomonas]

MULTISPECIES: aromatic amino acid aminotransferase

[Pseudomonas]

phenylacetate-CoA ligase [Pseudomonas putida]

MULTISPECIES: phenylacetic acid degradation protein

[Pseudomonas]

MULTISPECIES: phenylacetate-CoA oxygenase subunit

PaaJ [Pseudomonas]

MULTISPECIES: phenylacetic acid degradation protein

[Pseudomonas]

MULTISPECIES: phenylacetate-CoA oxygenase subunit

PaaB [Gammaproteobacteria]

MULTISPECIES: phenylacetate-CoA oxygenase subunit

PaaA [Pseudomonas]

MULTISPECIES: 2-(1,2-epoxy-1,2-dihydrophenyl)acetyl-

CoA isomerase [Pseudomonas]

enoyl-CoA hydratase [Pseudomonas putida]

MULTISPECIES: bifunctional aldehyde

dehydrogenase/enoyl-CoA hydratase [Pseudomonas]

enoyl-CoA hydratase [Pseudomonas putida]

enoyl-CoA hydratase [Pseudomonas putida]

MULTISPECIES: enoyl-CoA hydratase [Pseudomonas]

MULTISPECIES: 3-hydroxyacyl-CoA dehydrogenase

PaaC [Pseudomonas]
113

112

415

415

113

112

$98 \%$

$92 \%$

590

590

$99 \%$

590

590

$99 \%$

614

614

$97 \%$

345

345

$98 \%$

176

176

$93 \%$

258

258

$98 \%$

125

125

$94 \%$

456

456

$100 \%$

335

335

99\%

255

255

$96 \%$

724

724

$98 \%$

255

255

$96 \%$

255

255

$96 \%$

158

158

$99 \%$

387

$97 \%$ 
MULTISPECIES: 3-hydroxyacyl-CoA dehydrogenase

PaaC [Pseudomonas]

387

387

$97 \%$

MULTISPECIES: phenylacetic acid degradation protein

PaaD [Pseudomonas]

124

124

$93 \%$

MULTISPECIES: phenylacetic acid degradation protein

PaaD [Pseudomonas]

124

124

$93 \%$ 


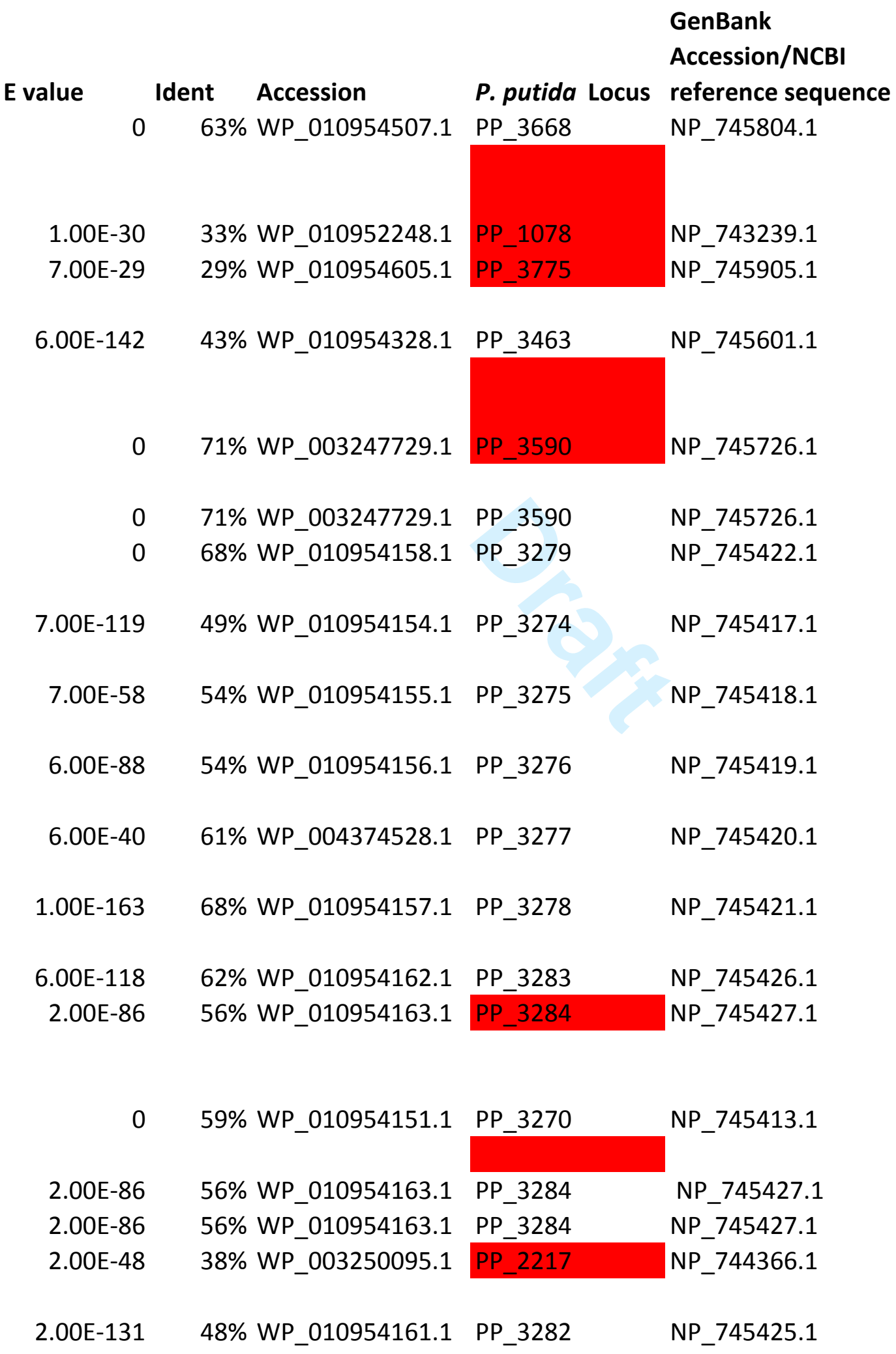




$\begin{array}{llll}2.00 E-131 & \text { 48\% WP_010954161.1 } & \text { PP_3282 } & \text { NP_745425.1 } \\ \text { 5.00E-38 } & \text { 47\% WP_010954160.1 } & \text { PP_3281 } & \text { NP_745424.1 } \\ 5.00 E-38 & \text { 47\% WP_010954160.1 } & \text { PP_3281 } & \text { NP_745424.1 }\end{array}$




\section{P. putida Gene Name} katG

peaE

$\mathrm{amaC}$

amaC

paak

paaE

paaD

paaC

paaB

paaA

paaG

paaF

paaZ

paaF

paaF

paaH 
paaH

paal

paal 
Results from P. putida KT2440 to E. coli K12 and E. coli K12 back to P. putida KT2440

\begin{tabular}{lcc} 
Pseudomonas Locus Tag E. coli Locus P.putida Locus \\
PP_3668 & b3942 & PP_3668 \\
PP_2932 & & \\
PP_2552 & b1756 & PP_1078 \\
PP_4983 & b1059 & PP_3775 \\
PP_3463 & b1385 & PP_3463 \\
PP_0967 & & \\
PP_1972 & b4054 & PP_3590 \\
PP_3590 & b4054 & PP_3590 \\
PP_3279 & b1398 & PP_3279 \\
PP_3274 & b1392 & PP_3274 \\
PP_3275 & b1391 & PP_3275 \\
PP_3276 & b1390 & PP_3276 \\
PP_3277 & b1389 & PP_3277 \\
PP_3278 & b1388 & PP_3278 \\
PP_3283 & b1394 & PP_3283 \\
PP_3726 & b1393 & PP_3284 \\
PP_3270 & b1387 & PP_3270 \\
PP_1845 & & \\
PP_2217 & b1393 & PP_3284 \\
PP_3284 & b1393 & PP_3284 \\
PP_4030 & b0036 & PP_2217 \\
PP_3282 & b1395 & PP_3282 \\
PP_3755 & b1395 & PP_3282 \\
PP_0244 & b1396 & PP_3281 \\
PP_3281 & b1396 & PP_3281 \\
\hline & &
\end{tabular}




\begin{tabular}{|c|c|}
\hline \multicolumn{2}{|c|}{ E. coli K-12 MG1655 prot } \\
\hline Query & \\
\hline E. coli gene name & Locus Tag \\
\hline katG & b3942 \\
\hline tynA & b1386 \\
\hline feaB & b1385 \\
\hline aspC & b0928 \\
\hline hisC & b2021 \\
\hline tyrB & b4054 \\
\hline paak & b1398 \\
\hline paal & b1396 \\
\hline paaA & b1388 \\
\hline paaB & b1389 \\
\hline paaC & b1390 \\
\hline paaD & b1391 \\
\hline paaE & b1392 \\
\hline paaG & b1394 \\
\hline paaZ & b1387 \\
\hline paal & b1397 \\
\hline paaF & b1393 \\
\hline paaH & b1395 \\
\hline
\end{tabular}


:ein sequences from ecocyc.com BLAST against $P$. putida KT2440 (taxid:160488)

Result

\section{Description}

catalase peroxidase [Pseudomonas putida]

Max Score Total Score

No Significant Similarity Found

MULTISPECIES: aldehyde dehydrogenase [Pseudomonas]

888

888

MULTISPECIES: aspartate aminotransferase [Pseudomonas]

415

415

373

373

MULTISPECIES: succinyldiaminopimelate transaminase [Pseudomonas]

61.2

61.2

MULTISPECIES: aromatic amino acid aminotransferase [Pseudomonas]

590

590

phenylacetate-CoA ligase [Pseudomonas putida]

614

614

MULTISPECIES: phenylacetic acid degradation protein PaaD

[Pseudomonas]

124

124

MULTISPECIES: phenylacetate-CoA oxygenase subunit PaaA

[Pseudomonas]

456

456

MULTISPECIES: phenylacetate-CoA oxygenase subunit PaaB

[Gammaproteobacteria]

125

125

MULTISPECIES: phenylacetic acid degradation protein [Pseudomonas]

258

258

MULTISPECIES: phenylacetate-CoA oxygenase subunit PaaJ

[Pseudomonas]

MULTISPECIES: phenylacetic acid degradation protein [Pseudomonas]

176

176

MULTISPECIES: 2-(1,2-epoxy-1,2-dihydrophenyl)acetyl-CoA isomerase

[Pseudomonas]

MULTISPECIES: bifunctional aldehyde dehydrogenase/enoyl-CoA

hydratase [Pseudomonas]

724

724

MULTISPECIES: acetyl-CoA acetyltransferase [Pseudomonas]

549

549

enoyl-CoA hydratase [Pseudomonas putida]

255

255

MULTISPECIES: 3-hydroxyacyl-CoA dehydrogenase PaaC [Pseudomonas] 


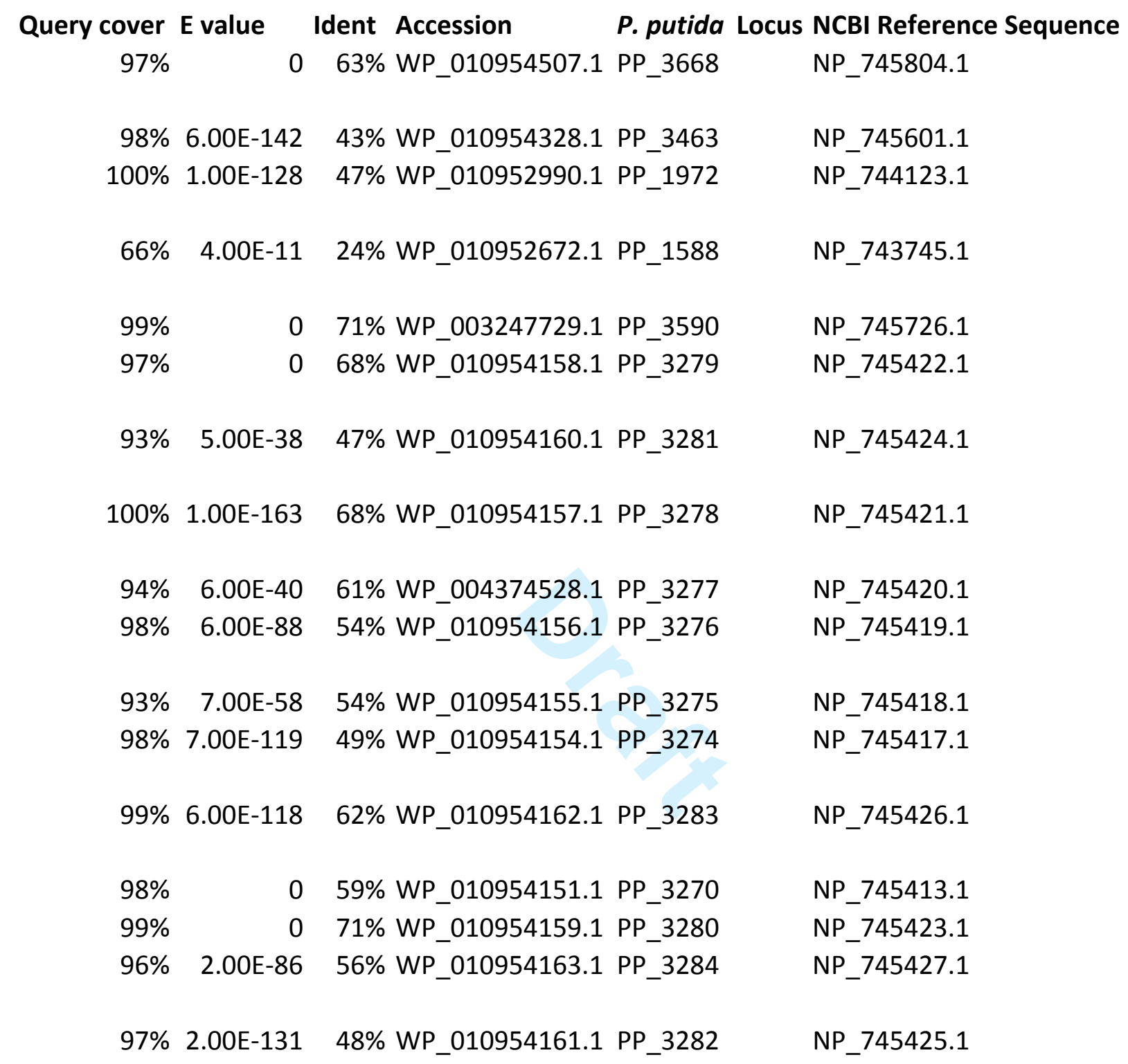




P. putida Gene Name
katG
peaE
tyrB
dapC
amaC
paaK
paal
paaA
paaB
paaC
paaD
paaE
paaG
paaZ
paaJ
paaF




\begin{tabular}{|c|c|c|}
\hline \multicolumn{3}{|c|}{ Results from $E$. col } \\
\hline \multicolumn{2}{|c|}{ Query } & \multirow[b]{2}{*}{$\begin{array}{l}\text { E. coli Locus } \\
\text { Initial query }\end{array}$} \\
\hline $\begin{array}{l}\text { P. putida gene } \\
\text { name }\end{array}$ & $\begin{array}{l}\text { P. putida } \\
\text { Locus }\end{array}$ & \\
\hline katG & PP_3668 & b3942 \\
\hline & & b1386 \\
\hline peaE & PP_3463 & b1385 \\
\hline tyrB & PP_1972 & b0928 \\
\hline dapC & PP_1588 & b2021 \\
\hline amaC & PP_3590 & b4054 \\
\hline paak & PP_3279 & b1398 \\
\hline paal & PP_3281 & b1396 \\
\hline paaA & PP_3278 & b1388 \\
\hline paaB & PP_3277 & b1389 \\
\hline paaC & PP_3276 & b1390 \\
\hline paaD & PP_3275 & b1391 \\
\hline paaE & PP_3274 & b1392 \\
\hline paaG & PP_3283 & b1394 \\
\hline paaZ & PP_3270 & b1387 \\
\hline paal & PP_3280 & b1397 \\
\hline paaF & PP_3284 & b1393 \\
\hline paaH & PP_3282 & b1395 \\
\hline
\end{tabular}


.0 stored as FASTA files BLAST against Escherichia coli MG1655 (taxid:511145)

Result

\section{Description}

MULTISPECIES: catalase-peroxidase [Proteobacteria]
Max Score Total Score cover 885

885

MULTISPECIES: NAD-dependent phenylacetaldehyde

dehydrogenase [Escherichia]

419

$41998 \%$

MULTISPECIES: aromatic amino acid aminotransferase

[Proteobacteria]

418

418

$99 \%$

MULTISPECIES: glutamate--pyruvate aminotransferase AlaC

[Proteobacteria]

141

$14198 \%$

MULTISPECIES: aromatic amino acid aminotransferase

[Proteobacteria]

MULTISPECIES: phenylacetate--CoA ligase [Enterobacteriaceae]

590

614

$59099 \%$

MULTISPECIES: acyl-CoA thioesterase [Enterobacteriaceae]

124

$61496 \%$

MULTISPECIES: 1,2-phenylacetyl-CoA epoxidase subunit A

[Enterobacteriaceae]*

456

$12489 \%$

MULTISPECIES: 1,2-phenylacetyl-CoA epoxidase subunit B

[Enterobacteriaceae]

125

MULTISPECIES: 1,2-phenylacetyl-CoA epoxidase subunit C

[Enterobacteriaceae]

258

MULTISPECIES: 1,2-phenylacetyl-CoA epoxidase

[Enterobacteriaceae]

176

350

350

$99 \%$

MULTISPECIES: 2-(1,2-epoxy-1,2-dihydrophenyl)acetyl-CoA

isomerase [Enterobacteriaceae]

308

308

$99 \%$

MULTISPECIES: bifunctional aldehyde dehydrogenase/enoyl-CoA

hydratase [Escherichia]

732

732

$98 \%$

MULTISPECIES: 3-oxoadipyl-CoA/3-oxo-5,6-dehydrosuberyl-CoA

thiolase [Enterobacteriaceae]

561

MULTISPECIES: 2,3-dehydroadipyl-CoA hydratase [Enterobacteriace

267

$56198 \%$

MULTISPECIES: 3-hydroxyadipyl-CoA dehydrogenase

[Enterobacteriaceae]

410

410

$96 \%$ 


\begin{tabular}{|c|c|c|c|c|}
\hline \multirow[t]{2}{*}{ E value } & Accession & \multirow{2}{*}{$\begin{array}{l}\text { E. coli Locus } \\
\text { b3942 }\end{array}$} & \multirow{2}{*}{$\begin{array}{l}\text { GenBank Accession } \\
\text { AAC76924.1 }\end{array}$} & \multirow{2}{*}{$\begin{array}{l}\text { E. coli } \\
\text { Name } \\
\text { katG }\end{array}$} \\
\hline & 63\% WP_001295695.1 & & & \\
\hline 8.00E-144 & 43\% WP_000138615.1 & b1385 & AAC74467.2 & feaB \\
\hline 3.00E-146 & 51\% WP_000486985.1 & b4054 & AAC77024.1 & tyrB \\
\hline 7.00E-39 & 28\% WP_000785931.1 & b2379 & AAC75438.1 & alaC \\
\hline 0 & 71\% WP_000486985.1 & b4054 & AAC77024.1 & tyrB \\
\hline 0 & 68\% WP_000632280.1 & b1398 & AAC74480.1 & paak \\
\hline $5.00 E-38$ & 47\% WP_000018413.1 & b1396 & AAC74478.1 & paal \\
\hline $1.00 E-163$ & 68\% WP_000191077.1 & b1388 & AAC74470.1 & paaA \\
\hline $5.00 \mathrm{E}-40$ & 61\% WP_000073393.1 & b1389 & AAC74471.1 & paaB \\
\hline $6.00 E-88$ & 54\% WP_001072837.1 & b1390 & AAC74472.1 & paaC \\
\hline $7.00 E-58$ & 54\% WP_001189201.1 & b1391 & AAC74473.4 & paaD \\
\hline 1.00E-120 & 49\% WP_000206388.1 & b1392 & AAC74474.1 & paaE \\
\hline $4.00 E-107$ & 62\% WP_000969784.1 & b1394 & AAC74476.1 & paaG \\
\hline 0 & 59\% WP_001186469.1 & b1387 & AAC74469.1 & paaZ \\
\hline 0 & 71\% WP_001206197.1 & b1397 & AAC74479.1 & paaJ \\
\hline 3.00E-91 & 56\% WP_001292353.1 & b1393 & AAC74475.1 & paaF \\
\hline 2.00E-140 & 48\% WP_000973360.1 & b1395 & AAC74477.1 & paaH \\
\hline
\end{tabular}


Results from E. coli K12 to P. putida KT2440 and P. putida KT2440 back to E. coli K12

$\begin{array}{llc}\text { E. coli Locus } & \text { P. putida Locus } \text { E. coli Locus } \\ \text { b3942 } & \text { PP_3668 } & \text { b3942 } \\ \text { b1386 } & & \\ \text { b1385 } & \text { PP_3463 } & \text { b1385 } \\ \text { b0928 } & \text { PP_1972 } & \text { b4054 } \\ \text { b2021 } & \text { PP_1588 } & \text { b2379 } \\ \text { b4054 } & \text { PP_3590 } & \text { b4054 } \\ \text { b1398 } & \text { PP_3279 } & \text { b1398 } \\ \text { b1396 } & \text { PP_3281 } & \text { b1396 } \\ \text { b1388 } & \text { PP_3278 } & \text { b1388 } \\ \text { b1389 } & \text { PP_3277 } & \text { b1389 } \\ \text { b1390 } & \text { PP_3276 } & \text { b1390 } \\ \text { b1391 } & \text { PP_3275 } & \text { b1391 } \\ \text { b1392 } & \text { PP_3274 } & \text { b1392 } \\ \text { b1394 } & \text { PP_3283 } & \text { b1394 } \\ \text { b1387 } & \text { PP_3270 } & \text { b1387 } \\ \text { b1397 } & \text { PP_3280 } & \text { b1397 } \\ \text { b1393 } & \text { PP_3284 } & \text { b1393 } \\ \text { b1395 } & \text { PP_3282 } & \text { b1395 }\end{array}$


Supplemental File 1: Bi-directional BLASTP from P. putida KT2440 to E. coli K-12 MG:

Overview:

\section{Bidirectional BLASTP (examplı}

BLASTP against
P. putida
E. coli genome
gene A

BLASTP against

P. putida E. coligenome gene B

Each tab contains the results from a BLASTP search

Tab \#

Tab 1

Tab 2

Tab 3

Tab 4

Tab 5

Tab 6

Tab 7

\section{Tab Title}

About File

$\mathrm{KT} 2440$ to $\mathrm{K} 12$

K12 back to KT2440

Bidirectional KT2440 to K12

K12 to KT2440

KT2440 back to K12

Bidirectional K12 to KT2440

\section{Contents}

Overview of Supplemental File 1

Contains the results from a BLASTP search using $P$. put E. coli K-12 MG1655 genes from the BLASTP search ag: Summary of the locus tag of the initial query gene (P. $p$ Contains the results from a BLASTP search using $E$. coli P. putida K-12 MG1655 genes from the BLASTP search Summary of the locus tag of the initial query gene ( $E . C$ 
e P. putida to E. coli)
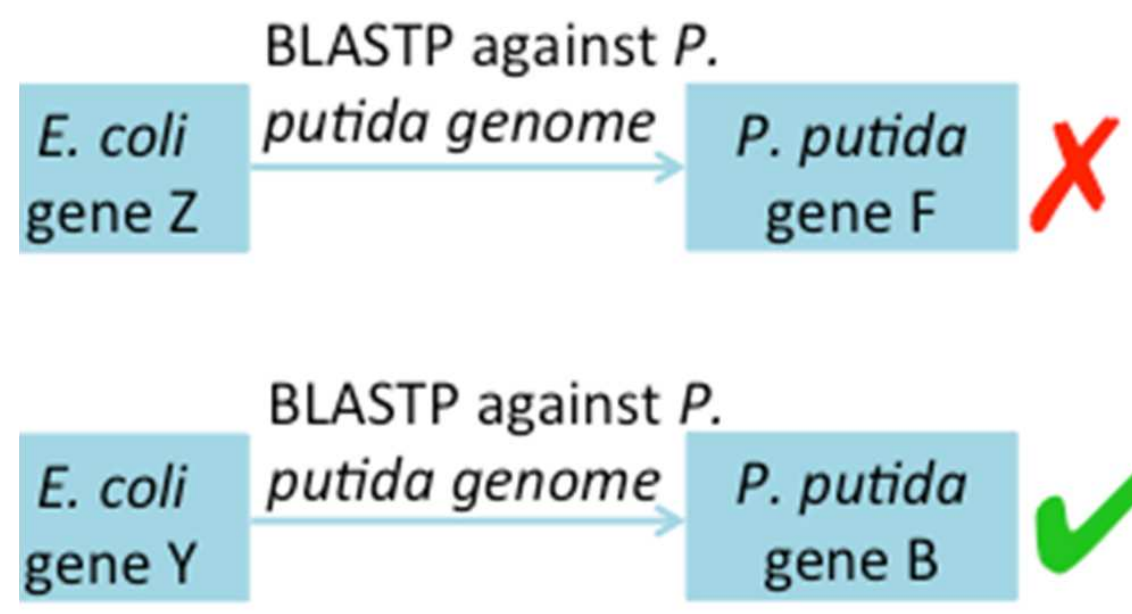

ida KT2440 genes as queries against the $E$. coli $\mathrm{K}-12 \mathrm{MG} 1655$ genome ainst the E. coli K-12 MG1655 genome were used in a BLASTP search against the P. putida KT244 Iutida $\mathrm{KT} 2440)$, the resulting hit from the E. coli $\mathrm{K}-12 \mathrm{MG} 1655$ genome and the resulting hit of $\mathrm{tl}$ K12-MG1655 genes as queries against the $P$. putida KT2440 genome against the $P$. putida $\mathrm{KT} 2440$ genome were used in a BLASTP search against the $E$. coli K-12 MG oli K-12 MG1655), the resulting hit from the P. putida KT2440 genome and the resulting hit of $\mathrm{tl}$ 
70 genome

רe $E$. coli K-12 MG1655 gene against the $P$. putida KT2440 genome 1655 genome

1e $P$. putida KT2440 gene against the $E$. coli K-12 MG1655 genome 
Pseudomonas putida KT2440 protein sequences from pseudomonas.com BLAST against $E$. coli M

Query

$P$. putida gene name

hisC

tyrB-1

tyrB-2

phaE

phal

phaH

paaB

paaA

phaB

phaL

fadB1x

PP_3283

PP_3726

\section{Result}

\section{Locus Tag Description}

PP_3668

PP_2932

MULTISPECIES: catalase peroxidase [Proteobacteria]

No Significant Similarity Found

MULTISPECIES: ABC transporter ATPase

PP_2552 [Proteobacteria]

MULTISPECIES: N-methyl-L-tryptophan oxidase

PP_4983 [Proteobacteria]

MULTISPECIES: NAD-dependent phenylacetaldehyde

PP_3463 dehydrogenase [Escherichia]

PP_0967 No Significant Similarity Found

MULTISPECIES: aromatic amino acid aminotransferase

PP_1972 [Proteobacteria]

MULTISPECIES: aromatic amino acid aminotransferase

PP_3590 [Proteobacteria]

MULTISPECIES: phenylacetate--CoA ligase

PP_3279 [Enterobacteriaceae]

MULTISPECIES: 1,2-phenylacetyl-CoA epoxidase

PP_3274 subunit E [Enterobacteriaceae]

MULTISPECIES: 1,2-phenylacetyl-CoA epoxidase

PP_3275 [Enterobacteriaceae]

MULTISPECIES: 1,2-phenylacetyl-CoA epoxidase

PP_3276 subunit C [Enterobacteriaceae]

MULTISPECIES: 1,2-phenylacetyl-CoA epoxidase

PP_3277 subunit B [Enterobacteriaceae]

MULTISPECIES: 1,2-phenylacetyl-CoA epoxidase

PP_3278 subunit A [Enterobacteriaceae]*

MULTISPECIES: 2-(1,2-epoxy-1,2-dihydrophenyl)acetyl-

CoA isomerase [Enterobacteriaceae]

MULTISPECIES: 2,3-dehydroadipyl-CoA hydratase

[Enterobacteriaceae]

MULTISPECIES: phenylacetic acid degradation

PP_3270 bifunctional protein PaaZ [Escherichia]

PP_1845 No Significant Similarity Found

MULTISPECIES: 2,3-dehydroadipyl-CoA hydratase

PP_2217 [Enterobacteriaceae]

MULTISPECIES: 2,3-dehydroadipyl-CoA hydratase

PP_3284 [Enterobacteriaceae] 


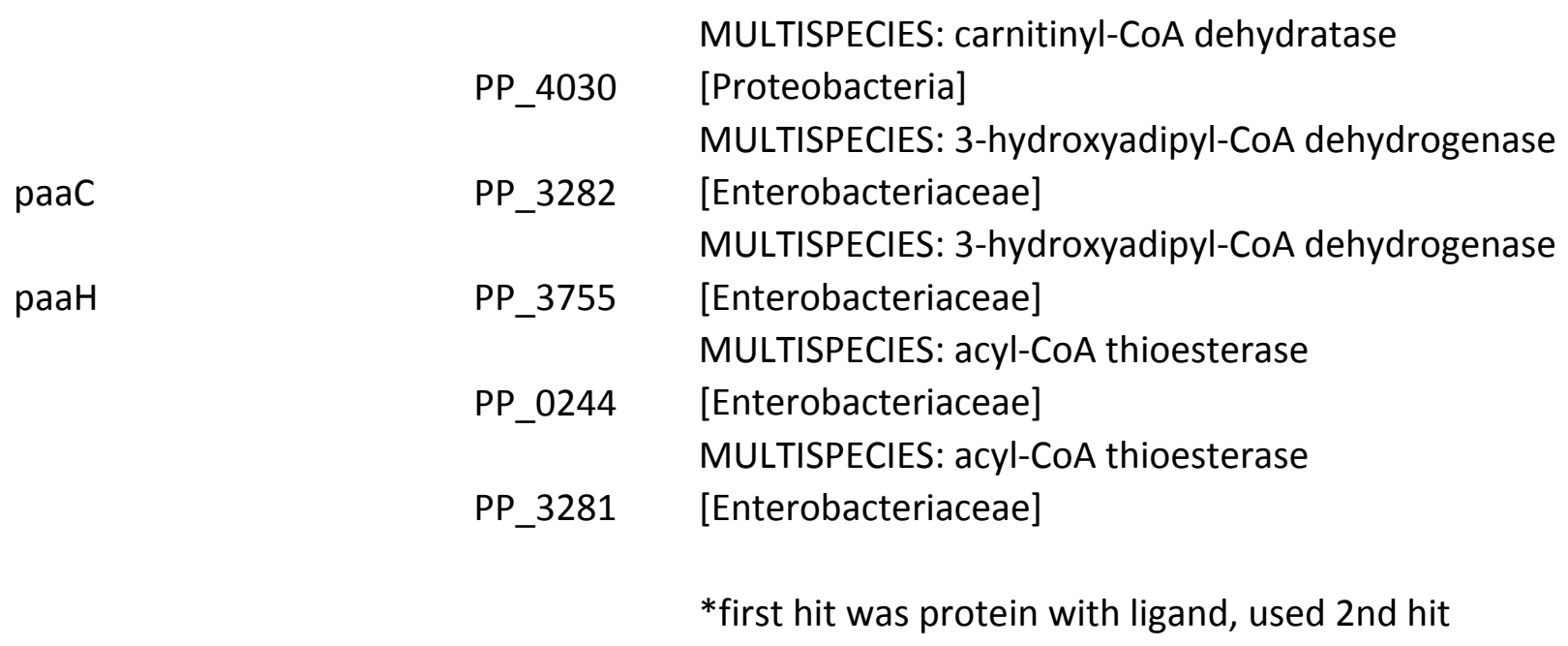




\section{G1655 (taxid:511145)}

\begin{tabular}{|c|c|c|c|c|c|c|}
\hline Max Score & Total Score & Query cover & E value & Ident & Accession & E. coli Locus \\
\hline 885 & 885 & $99 \%$ & 0 & $63 \%$ & WP_001295695.1 & b3942 \\
\hline 26.2 & 26.2 & $9 \%$ & 5.4 & $37 \%$ & WP_001300558.1 & b1756 \\
\hline 30.4 & 30.4 & $4 \%$ & 0.45 & $52 \%$ & WP_000872833.1 & b1059 \\
\hline 419 & 419 & $98 \%$ & $8.00 \mathrm{E}-144$ & $43 \%$ & WP_000138615.1 & b1385 \\
\hline 418 & 418 & $99 \%$ & $3.00 \mathrm{E}-146$ & $51 \%$ & 6P_000486985.1 & b4054 \\
\hline 590 & 590 & $99 \%$ & $0.00 E+00$ & $71 \%$ & WP_000486985.1 & b4054 \\
\hline 614 & 614 & $96 \%$ & 0 & $68 \%$ & WP_000632280.1 & b1398 \\
\hline 350 & 350 & $99 \%$ & $1.00 \mathrm{E}-120$ & $49 \%$ & WP_000206388.1 & b1392 \\
\hline 176 & 176 & $88 \%$ & $7.00 \mathrm{E}-58$ & $54 \%$ & WP_001189201.1 & b1391 \\
\hline 258 & 258 & $98 \%$ & 2.00E-91 & $54 \%$ & WP_001072837.1 & b1390 \\
\hline 125 & 125 & $96 \%$ & $5.00 E-40$ & $61 \%$ & WP_000073393.1 & b1389 \\
\hline 456 & 456 & $94 \%$ & $1.00 \mathrm{E}-163$ & $68 \%$ & WP_000191077.1 & b1388 \\
\hline 308 & 308 & $99 \%$ & 4.00E-107 & $62 \%$ & WP_000969784.1 & b1394 \\
\hline 142 & 142 & $98 \%$ & $2.00 E-42$ & $36 \%$ & WP_001292353.1 & b1393 \\
\hline 732 & 732 & $98 \%$ & 0 & $59 \%$ & WP_001186469.1 & b1387 \\
\hline 200 & 200 & $95 \%$ & 7.00E-65 & $43 \%$ & WP_001292353.1 & b1393 \\
\hline 267 & 267 & $96 \%$ & $3.00 E-91$ & $56 \%$ & WP_001292353.1 & b1393 \\
\hline
\end{tabular}




$\begin{array}{rrrrrr}128 & 128 & 91 \% & 2.00 \mathrm{E}-36 & 34 \% \mathrm{WP} 001295419.1 & \text { b0036 } \\ 410 & 410 & 96 \% & 2.00 \mathrm{E}-140 & 48 \% \text { WP_000973360.1 } & \text { b1395 } \\ 219 & 303 & 100 \% & 3.00 \mathrm{E}-69 & 41 \% \text { WP_000973360.1 } & \text { b1395 } \\ 61.2 & 61.2 & 91 \% & 8.00 \mathrm{E}-14 & 36 \% \text { WP_000018413.1 } & \text { b1396 } \\ 124 & 124 & 89 \% & 5.00 \mathrm{E}-38 & 47 \% \text { WP_000018413.1 } & \text { b1396 }\end{array}$




\section{GenBank Accession E. coli Gene Name}

AAC76924.1 katG

$\begin{array}{ll}\text { AAC74826.1 } & \text { ynjD } \\ \text { AAC74143.1 } & \text { solA } \\ \text { AAC74467.2 } & \text { feaB }\end{array}$

AAC77024.1 tyrB

AAC77024.1 tyrB

AAC74480.1 paak

AAC74474.1 paaE

AAC74473.4 paaD

AAC74472.1 paaC

AAC74471.1 раaB

AAC74470.1 paaA

AAC74476.1 paaG

AAC74475.1 paaF

AAC74469.1 paaZ

AAC74475.1 paaF

AAC74475.1 paaF 
AAC73147.2 caiD

AAC74477.1 paaH

AAC74477.1 paaH

AAC74478.1 paal

AAC74478.1 paal 
Reverse Blast of $E$. coli $\mathrm{K}-12$ gene results fror

Query

P. putida Locus

E. coli gene name $E$. coli Locus Initial Query

katG

b3942 PP_3668

PP_2932

ynjD

b1756

PP_2552

solA

b1059

PP_4983

feaB

b1385

PP_3463

PP_0967

tyrB

b4054

PP_1972

tyrB

b4054

PP_3590

paak

b1398

PP_3279

paaE

b1392

PP_3274

paaD

b1391

PP_3275

paaC

b1390

PP_3276

paaB

b1389

PP_3277

paaA

b1388

PP_3278

paaG

b1394

PP_3283

paaF

b1393

PP_3726

paaZ

b1387

PP_3270

PP_1845

paaF

b1393

PP_2217

paaF

b1393

PP_3284

b0036

PP_4030

paaH

b1395

PP_3282 


$\begin{array}{lll}\text { paaH } & \text { b1395 } & \text { PP_3755 } \\ \text { paal } & \text { b1396 } & \text { PP_0244 } \\ \text { paal } & \text { b1396 } & \text { PP_3281 }\end{array}$


n P. putida KT2440 to E. coli K12 against $P$. putida KT2440 (taxid:160488)

Result

\section{Description}

catalase peroxidase [Pseudomonas putida]
Max Score Total Score Query cover 888

888

$97 \%$

[Pseudomonas]

MULTISPECIES: aromatic amino acid aminotransferase [Pseudomonas]

MULTISPECIES: aromatic amino acid aminotransferase

[Pseudomonas]

phenylacetate-CoA ligase [Pseudomonas putida]

MULTISPECIES: phenylacetic acid degradation protein

[Pseudomonas]

MULTISPECIES: phenylacetate-CoA oxygenase subunit

PaaJ [Pseudomonas]

MULTISPECIES: phenylacetic acid degradation protein

[Pseudomonas]

MULTISPECIES: phenylacetate-CoA oxygenase subunit

PaaB [Gammaproteobacteria]

MULTISPECIES: phenylacetate-CoA oxygenase subunit

PaaA [Pseudomonas]

MULTISPECIES: 2-(1,2-epoxy-1,2-dihydrophenyl)acetyl-

CoA isomerase [Pseudomonas]

enoyl-CoA hydratase [Pseudomonas putida]

MULTISPECIES: bifunctional aldehyde

dehydrogenase/enoyl-CoA hydratase [Pseudomonas]

enoyl-CoA hydratase [Pseudomonas putida]

enoyl-CoA hydratase [Pseudomonas putida]

MULTISPECIES: enoyl-CoA hydratase [Pseudomonas]

MULTISPECIES: 3-hydroxyacyl-CoA dehydrogenase

PaaC [Pseudomonas]
113

112

415

415

113

112

$98 \%$

$92 \%$

590

590

$99 \%$

590

590

$99 \%$

614

614

$97 \%$

345

345

$98 \%$

176

176

$93 \%$

258

258

$98 \%$

125

125

$94 \%$

456

456

$100 \%$

335

335

99\%

255

255

$96 \%$

724

724

$98 \%$

255

255

$96 \%$

255

255

$96 \%$

158

158

$99 \%$

387

$97 \%$ 
MULTISPECIES: 3-hydroxyacyl-CoA dehydrogenase

PaaC [Pseudomonas]

387

387

$97 \%$

MULTISPECIES: phenylacetic acid degradation protein

PaaD [Pseudomonas]

124

124

$93 \%$

MULTISPECIES: phenylacetic acid degradation protein

PaaD [Pseudomonas]

124

124

$93 \%$ 


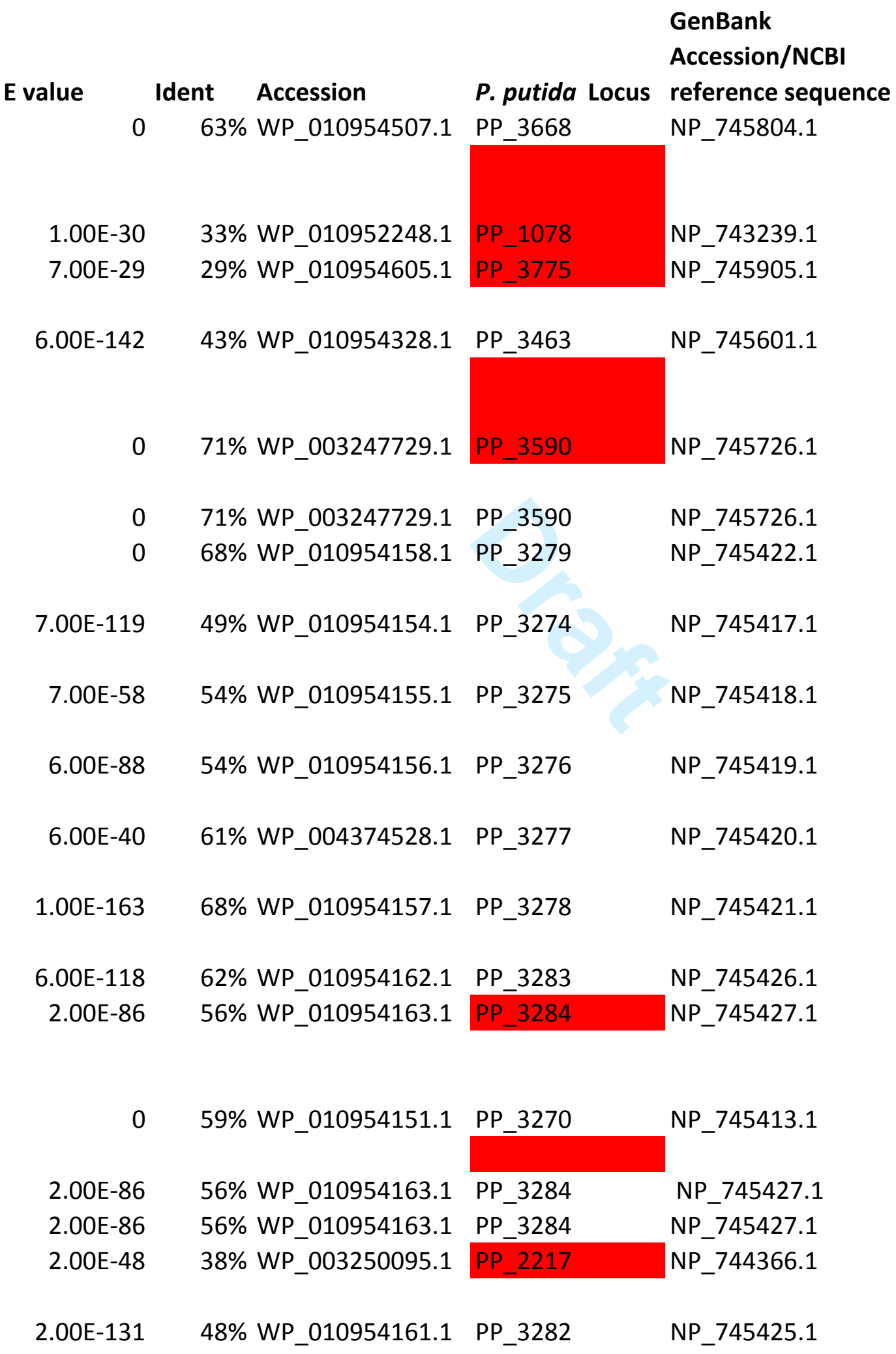




$\begin{array}{llll}\text { 2.00E-131 } & \text { 48\% WP_010954161.1 } & \text { PP_3282 } & \text { NP_745425.1 } \\ \text { 5.00E-38 } & \text { 47\% WP_010954160.1 } & \text { PP_3281 } & \text { NP_745424.1 } \\ 5.00 E-38 & \text { 47\% WP_010954160.1 } & \text { PP_3281 } & \text { NP_745424.1 }\end{array}$




\section{P. putida Gene Name} katG

peaE

$\mathrm{amaC}$

amaC

paak

paaE

paaD

paaC

paaB

paaA

paaG

paaF

paaZ

paaF

paaF

paaH 
paaH

paal

paal 
Results from $P$. putida KT2440 to E. coli K12 and E. coli K12 back to $P$. putida KT2440

\begin{tabular}{lcc} 
Pseudomonas Locus Tag E. coli Locus P.putida Locus \\
PP_3668 & b3942 & PP_3668 \\
PP_2932 & & \\
PP_2552 & b1756 & PP_1078 \\
PP_4983 & b1059 & PP_3775 \\
PP_3463 & b1385 & PP_3463 \\
PP_0967 & & \\
PP_1972 & b4054 & PP_3590 \\
PP_3590 & b4054 & PP_3590 \\
PP_3279 & b1398 & PP_3279 \\
PP_3274 & b1392 & PP_3274 \\
PP_3275 & b1391 & PP_3275 \\
PP_3276 & b1390 & PP_3276 \\
PP_3277 & b1389 & PP_3277 \\
PP_3278 & b1388 & PP_3278 \\
PP_3283 & b1394 & PP_3283 \\
PP_3726 & b1393 & PP_3284 \\
PP_3270 & b1387 & PP_3270 \\
PP_1845 & & \\
PP_2217 & b1393 & PP_3284 \\
PP_3284 & b1393 & PP_3284 \\
PP_4030 & b0036 & PP_2217 \\
PP_3282 & b1395 & PP_3282 \\
PP_3755 & b1395 & PP_3282 \\
PP_0244 & b1396 & PP_3281 \\
PP_3281 & PP_3281 \\
\hline
\end{tabular}




\begin{tabular}{|c|c|}
\hline \multicolumn{2}{|c|}{ E. coli K-12 MG1655 pro } \\
\hline Query & \\
\hline E. coli gene name & Locus Tag \\
\hline katG & b3942 \\
\hline tynA & b1386 \\
\hline feaB & b1385 \\
\hline aspC & b0928 \\
\hline hisC & b2021 \\
\hline tyrB & b4054 \\
\hline paak & b1398 \\
\hline paal & b1396 \\
\hline paaA & b1388 \\
\hline paaB & b1389 \\
\hline paaC & b1390 \\
\hline paaD & b1391 \\
\hline paaE & b1392 \\
\hline paaG & b1394 \\
\hline paaZ & b1387 \\
\hline paaJ & b1397 \\
\hline paaF & b1393 \\
\hline paaH & b1395 \\
\hline
\end{tabular}


:ein sequences from ecocyc.com BLAST against $P$. putida KT2440 (taxid:160488)

Result

\section{Description}

catalase peroxidase [Pseudomonas putida]

Max Score Total Score

No Significant Similarity Found

MULTISPECIES: aldehyde dehydrogenase [Pseudomonas]

888

888

MULTISPECIES: aspartate aminotransferase [Pseudomonas]

415

415

373

373

MULTISPECIES: succinyldiaminopimelate transaminase [Pseudomonas]

61.2

61.2

MULTISPECIES: aromatic amino acid aminotransferase [Pseudomonas]

590

590

phenylacetate-CoA ligase [Pseudomonas putida]

614

614

MULTISPECIES: phenylacetic acid degradation protein PaaD

[Pseudomonas]

124

124

MULTISPECIES: phenylacetate-CoA oxygenase subunit PaaA

[Pseudomonas]

456

456

MULTISPECIES: phenylacetate-CoA oxygenase subunit PaaB

[Gammaproteobacteria]

125

125

MULTISPECIES: phenylacetic acid degradation protein [Pseudomonas]

258

258

MULTISPECIES: phenylacetate-CoA oxygenase subunit PaaJ

[Pseudomonas]

MULTISPECIES: phenylacetic acid degradation protein [Pseudomonas]

176

176

MULTISPECIES: 2-(1,2-epoxy-1,2-dihydrophenyl)acetyl-CoA isomerase

[Pseudomonas]

MULTISPECIES: bifunctional aldehyde dehydrogenase/enoyl-CoA

hydratase [Pseudomonas]

724

724

MULTISPECIES: acetyl-CoA acetyltransferase [Pseudomonas]

549

549

enoyl-CoA hydratase [Pseudomonas putida]

255

255

MULTISPECIES: 3-hydroxyacyl-CoA dehydrogenase PaaC [Pseudomonas] 


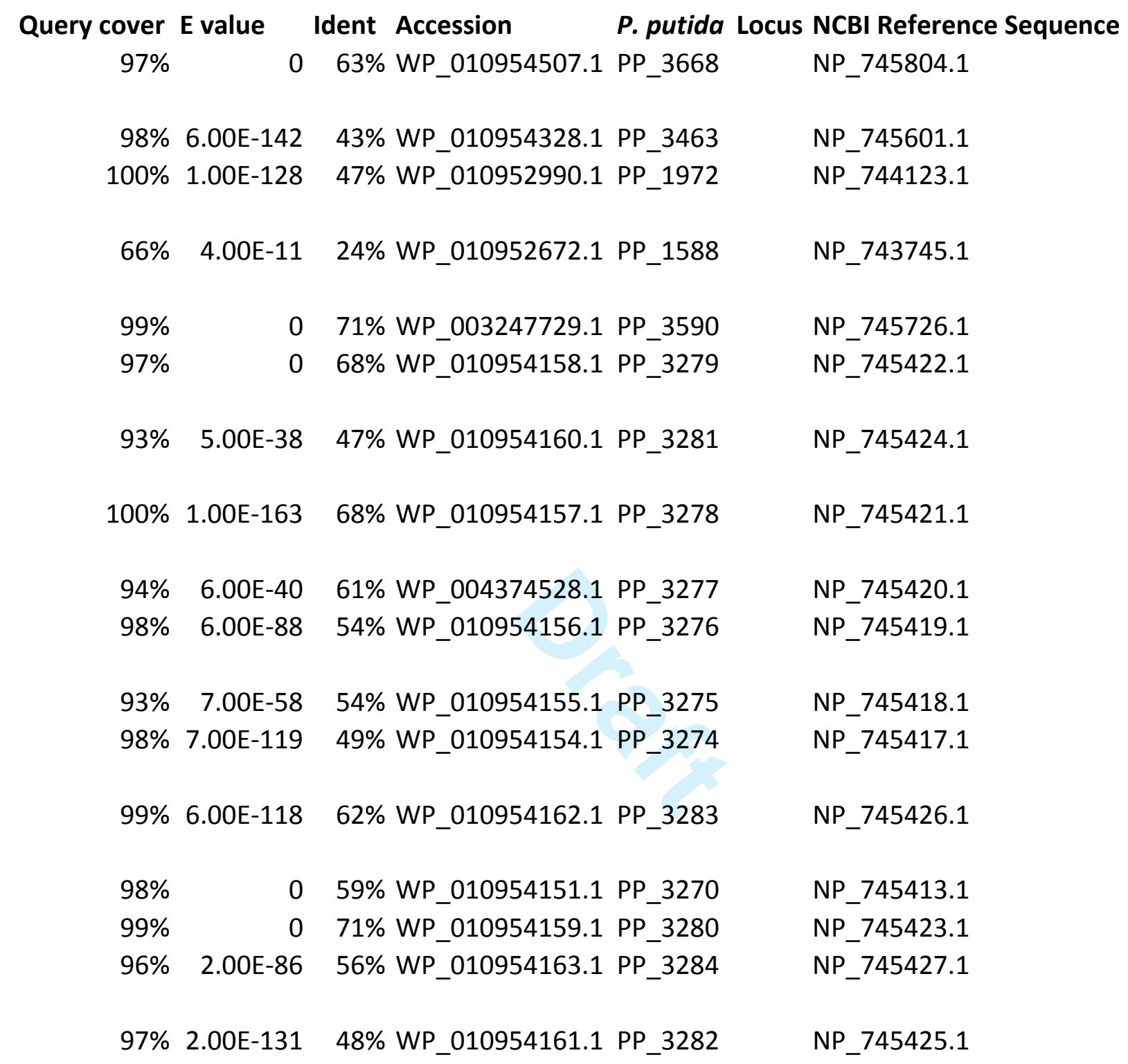




P. putida Gene Name
katG
peaE
tyrB
dapC
amaC
paaK
paal
paaA
paaB
paaC
paaD
paaE
paaG
paaZ
paaJ
paaF




\begin{tabular}{|c|c|c|}
\hline \multicolumn{3}{|c|}{ Results from $E$. col } \\
\hline \multicolumn{2}{|c|}{ Query } & \multirow[b]{2}{*}{$\begin{array}{l}\text { E. coli Locus } \\
\text { Initial query }\end{array}$} \\
\hline $\begin{array}{l}\text { P. putida gene } \\
\text { name }\end{array}$ & $\begin{array}{l}\text { P. putida } \\
\text { Locus }\end{array}$ & \\
\hline katG & PP_3668 & b3942 \\
\hline & & b1386 \\
\hline peaE & PP_3463 & b1385 \\
\hline tyrB & PP_1972 & b0928 \\
\hline dapC & PP_1588 & b2021 \\
\hline amaC & PP_3590 & b4054 \\
\hline paak & PP_3279 & b1398 \\
\hline paal & PP_3281 & b1396 \\
\hline paaA & PP_3278 & b1388 \\
\hline paaB & PP_3277 & b1389 \\
\hline paaC & PP_3276 & b1390 \\
\hline paaD & PP_3275 & b1391 \\
\hline paaE & PP_3274 & b1392 \\
\hline paaG & PP_3283 & b1394 \\
\hline paaZ & PP_3270 & b1387 \\
\hline paal & PP_3280 & b1397 \\
\hline paaF & PP_3284 & b1393 \\
\hline paaH & PP_3282 & b1395 \\
\hline
\end{tabular}


.0 stored as FASTA files BLAST against Escherichia coli MG1655 (taxid:511145)

Result

\section{Description}

MULTISPECIES: catalase-peroxidase [Proteobacteria]
Max Score Total Score cover 885

885

MULTISPECIES: NAD-dependent phenylacetaldehyde

dehydrogenase [Escherichia]

419

$41998 \%$

MULTISPECIES: aromatic amino acid aminotransferase

[Proteobacteria]

418

418

$99 \%$

MULTISPECIES: glutamate--pyruvate aminotransferase AlaC

[Proteobacteria]

141

$14198 \%$

MULTISPECIES: aromatic amino acid aminotransferase

[Proteobacteria]

MULTISPECIES: phenylacetate--CoA ligase [Enterobacteriaceae]

590

614

$59099 \%$

MULTISPECIES: acyl-CoA thioesterase [Enterobacteriaceae]

124

$61496 \%$

MULTISPECIES: 1,2-phenylacetyl-CoA epoxidase subunit A

[Enterobacteriaceae]*

456

$12489 \%$

MULTISPECIES: 1,2-phenylacetyl-CoA epoxidase subunit B

[Enterobacteriaceae]

125

MULTISPECIES: 1,2-phenylacetyl-CoA epoxidase subunit C

[Enterobacteriaceae]

258

MULTISPECIES: 1,2-phenylacetyl-CoA epoxidase

[Enterobacteriaceae]

176

350

350

$99 \%$

MULTISPECIES: 2-(1,2-epoxy-1,2-dihydrophenyl)acetyl-CoA

isomerase [Enterobacteriaceae]

308

308

$99 \%$

MULTISPECIES: bifunctional aldehyde dehydrogenase/enoyl-CoA

hydratase [Escherichia]

732

732

$98 \%$

MULTISPECIES: 3-oxoadipyl-CoA/3-oxo-5,6-dehydrosuberyl-CoA

thiolase [Enterobacteriaceae]

561

MULTISPECIES: 2,3-dehydroadipyl-CoA hydratase [Enterobacteriace

267

$56198 \%$

MULTISPECIES: 3-hydroxyadipyl-CoA dehydrogenase

[Enterobacteriaceae]

410

410

$96 \%$ 


\begin{tabular}{|c|c|c|c|c|}
\hline \multirow[t]{2}{*}{ E value } & Accession & \multirow{2}{*}{$\begin{array}{l}\text { E. coli Locus } \\
\text { b3942 }\end{array}$} & \multirow{2}{*}{$\begin{array}{l}\text { GenBank Accession } \\
\text { AAC76924.1 }\end{array}$} & \multirow{2}{*}{$\begin{array}{l}\text { E. coli } \\
\text { Name } \\
\text { katG }\end{array}$} \\
\hline & 63\% WP_001295695.1 & & & \\
\hline 8.00E-144 & 43\% WP_000138615.1 & b1385 & AAC74467.2 & feaB \\
\hline 3.00E-146 & 51\% WP_000486985.1 & b4054 & AAC77024.1 & tyrB \\
\hline 7.00E-39 & 28\% WP_000785931.1 & b2379 & AAC75438.1 & alaC \\
\hline 0 & 71\% WP_000486985.1 & b4054 & AAC77024.1 & tyrB \\
\hline 0 & 68\% WP_000632280.1 & b1398 & AAC74480.1 & paak \\
\hline $5.00 E-38$ & 47\% WP_000018413.1 & b1396 & AAC74478.1 & paal \\
\hline $1.00 E-163$ & 68\% WP_000191077.1 & b1388 & AAC74470.1 & paaA \\
\hline $5.00 \mathrm{E}-40$ & 61\% WP_000073393.1 & b1389 & AAC74471.1 & paaB \\
\hline $6.00 E-88$ & 54\% WP_001072837.1 & b1390 & AAC74472.1 & paaC \\
\hline $7.00 E-58$ & 54\% WP_001189201.1 & b1391 & AAC74473.4 & paaD \\
\hline 1.00E-120 & 49\% WP_000206388.1 & b1392 & AAC74474.1 & paaE \\
\hline $4.00 E-107$ & 62\% WP_000969784.1 & b1394 & AAC74476.1 & paaG \\
\hline 0 & 59\% WP_001186469.1 & b1387 & AAC74469.1 & paaZ \\
\hline 0 & 71\% WP_001206197.1 & b1397 & AAC74479.1 & paaJ \\
\hline 3.00E-91 & 56\% WP_001292353.1 & b1393 & AAC74475.1 & paaF \\
\hline 2.00E-140 & 48\% WP_000973360.1 & b1395 & AAC74477.1 & paaH \\
\hline
\end{tabular}


Results from E. coli K12 to P. putida KT2440 and P. putida KT2440 back to E. coli K12

$\begin{array}{llc}\text { E. coli Locus } & \text { P. putida Locus } \text { E. coli Locus } \\ \text { b3942 } & \text { PP_3668 } & \text { b3942 } \\ \text { b1386 } & & \\ \text { b1385 } & \text { PP_3463 } & \text { b1385 } \\ \text { b0928 } & \text { PP_1972 } & \text { b4054 } \\ \text { b2021 } & \text { PP_1588 } & \text { b2379 } \\ \text { b4054 } & \text { PP_3590 } & \text { b4054 } \\ \text { b1398 } & \text { PP_3279 } & \text { b1398 } \\ \text { b1396 } & \text { PP_3281 } & \text { b1396 } \\ \text { b1388 } & \text { PP_3278 } & \text { b1388 } \\ \text { b1389 } & \text { PP_3277 } & \text { b1389 } \\ \text { b1390 } & \text { PP_3276 } & \text { b1390 } \\ \text { b1391 } & \text { PP_3275 } & \text { b1391 } \\ \text { b1392 } & \text { PP_3274 } & \text { b1392 } \\ \text { b1394 } & \text { PP_3283 } & \text { b1394 } \\ \text { b1387 } & \text { PP_3270 } & \text { b1387 } \\ \text { b1397 } & \text { PP_3280 } & \text { b1397 } \\ \text { b1393 } & \text { PP_3284 } & \text { b1393 } \\ \text { b1395 } & \text { PP_3282 } & \text { b1395 }\end{array}$


Supplemental File 2: Identification of putative genes of the phenylalanine catabolic pathway in 3 CF pathogens by bi-directional BLASTP searches using $P$. putida KT2440 and $E$. coli K-12 MG1655 genes against the genome of each CF pathogen

Overview:

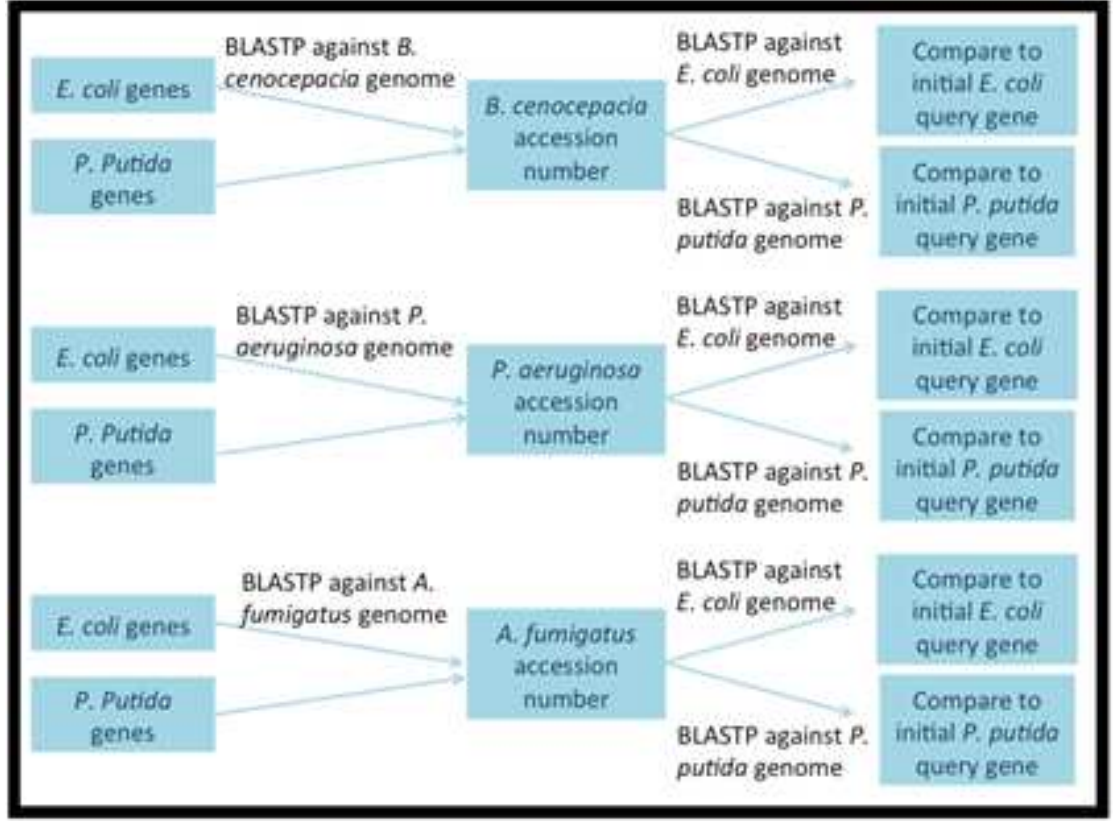

Using the KEGG database the phenylalanine catabolic pathway for each CF strain was elucidated. (See CF Strain Pathways Tab)

Steps where no gene has been identified by KEGG were selected for further investigation (Highlighted Red in CF Strain Pathways Tab).

Create a FASTA File (one for each strain) containing the amino acid sequences for the genes (from P. putida KT2440 and E. coli K-12 MG1655 responsible for the steps with genes absent in eacl These FASTA files were used to BLAST against each genome

The accession number of the resulting hits from each genome was used as a query in a BLASTP search back against the initial genome (P. putida or E. coli)

If the locus tag of the initial query matched the locus tag returned from the bidirectional BLASTP search, it was considered a putative gene for the step.

BLAST against $P$. aeruginosa

$P$. aeruginosa back to genome The accession numbers of the genes identified by BLAST against $P$. aeruginos $a$ were used as queries back against the initial $P$. putid

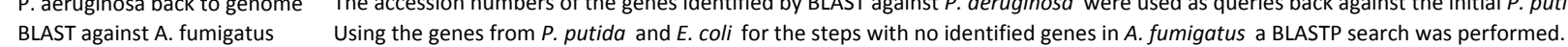

A. fumigatus back to genome The accession numbers of the genes identified by BLAST against A. fumigatus were used as queries back against the initial P. putida or E. coli genome 


\begin{tabular}{|c|c|c|c|c|c|}
\hline Step in Pathway & Locus Tag(s) in P. putida & Locus Tag(s) in E. coli & B. cenocepacia & P. aeruginosa & A. fumigatus \\
\hline & PP_0967 & b0928 & BCAL0313 & PA2531 & AFUA_1G04160 \\
\hline & PP_1972 & b2021 & BCAL0797 & PA3165 & AFUA_2G09650 \\
\hline & PP_3590 & b4054 & BCAL2303 & PA4447 & AFUA_4G10410 \\
\hline & & & BCAM1478 & PA0870 & AFUA_-6G02490 \\
\hline & & & & PA3139 & AFUA_1G14090 \\
\hline & & & & & AFUA_2G13630 \\
\hline & PP_2552 & & & & AFUA_3G02240 \\
\hline & PP_3668 & b3942 & BCAL3299 & & AFUA_8G01670 \\
\hline & & & BCAM2107 & & \\
\hline & PP_4983 & b1386 & BCAM0112 & PA0421 & AFUA_3G00100 \\
\hline & & & BCAM0123 & & AFUA_1G13440 \\
\hline & & & & & AFUA_3G00680 \\
\hline & & & & & AFUA_-3G14590 \\
\hline & & & & & AFUA_5G01470 \\
\hline & & & & & AFUA_5G07360 \\
\hline & & & & & AFUA_7G04180 \\
\hline & & & & & AFUA_-7G08470 \\
\hline & PP_2932 & & \begin{tabular}{|l} 
BCAL0148 \\
\end{tabular} & PA0202 & AFUA_1G14880 \\
\hline & & & BCAM0538 & PA0704 & AFUA_5G00470 \\
\hline & & & BCAM0641 & PA3366 & AFUA_5G09140 \\
\hline & & & BCAM1315 & PA4163 & AFUA_6G08000 \\
\hline & & & BCAM1544 & PA4342 & AFUA_6G14410 \\
\hline & & & BCAM1828 & & AFUA_8G05220 \\
\hline & PP_3463 & b1385 & BCAM1979 & PA4073 & \\
\hline & & & BCAM2298 & & \\
\hline & & & & & \\
\hline & PP_0244 & b1396 & BCAL0405 & PA5202 & \\
\hline & PP_3281 & & & & \\
\hline 10 & PP_3279 & b1398 & \begin{tabular}{|l|} 
BCAL0404 \\
\end{tabular} & & \\
\hline & & & BCAM1711 & & \\
\hline 11 & PP_3274 & b1388 & BCAL0212 & & \\
\hline & PP_3275 & b1389 & BCAL0213 & & \\
\hline & PP_3276 & b1390 & BCAL0214 & & \\
\hline & PP_3277 & b1391 & BCAL0215 & & \\
\hline & PP_3278 & b1392 & BCAL0216 & & \\
\hline 12 & PP_3283 & b1394 & \begin{tabular}{|l|} 
BCAL0406 \\
\end{tabular} & PA4980 & \\
\hline & PP_3726 & & & & \\
\hline 13 & PP_3270 & b1387 & & & \\
\hline 14 & PP_3270 & b1387 & & & \\
\hline 15 & PP_3283 & b1394 & BCAL0406 & PA4980 & \\
\hline & PP_3726 & b1397 & & & \\
\hline 16 & PP_1845 & b1393 & BCAL0409 & PA1748 & \\
\hline & PP_2217 & & BCAL0687 & PA1821 & \\
\hline & PP_3284 & & BCAM1632 & PA3426 & \\
\hline & PP_4030 & & BCAM1634 & & \\
\hline 17 & PP_3282 & b1395 & BCAM0656 & PA1628 & AFUA_4G04410 \\
\hline & PP_3755 & & BCAM1712 & PA3590 & \\
\hline & & & BCAS0607 & & \\
\hline 18 & 3 PP_3280 & b1397 & & & \\
\hline
\end{tabular}


Step in Pathway Locus Tag(s) in P. putida Locus Tag(s) in E. coll PP_2552

PP_3270

Absent

13 and 14
B. cenocepacia using e value threshold $10^{\wedge-8}$

MULTISPECIES: aldehyde dehydrogenase [Burkholderia cepacia complex]

MULTISPECIES: aldehyde dehydrogenase [Burkholderia cepacia complex]

betaine aldehyde dehydrogenase [Burkholderia cenocepacia J2315]

MULTISPECIES: 5-carboxymethy-2-hydroxymuconate semialdehyde dehydrogenase [Burkholderia cepacia complex]

MULTISPECLS. aldehyde dehydrogenase (BUH holderia cepacia complex],

MULTISPECIES: aldehyde dehydrogenase [Burkholderia cepacia complex]

MULTISPECIES: phosphonoacetaldehyde dehydrogenase [Burkholderia cepacia complex]

MULTSPECLS: phosphonoacetaldehyde dehydrogenase [Burkholderia cepacia complex] MUain A, The Crystal Structure of Gamma-glutamyl-gamma-aminobutyrald
MULISPECIES: aldehyde dehydrogenase [Burkholderia cepacia complex]

MULTISPECIES: gamma-glutamyl-gamma-aminobutyraldehyde dehydrogenase [Burkholderia cepacia complex]

(acylating) [Burkholderia cepacia complex]

is

dentrogenase [Burkholderia cepacia complex]

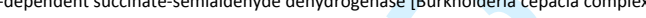

MULTISPECIES adehye dehydrogenase [Burkholderia cepacia complex]

MULTIIPECIES: phosphonoacetaldehyde dehydrogenase [Burkholderia cepacia complex]

MULTISPECISS phosphe

MULTISPECIES: phosphonoacetaldehyde dehydrogenase [Burkholderia cepacia complex]

putative NADP-dependent glyceraldehyde-3-phosphate dehydrogenase [Burkholderia cenocepacia J2315]

MULTISPECIES: aldehyde dehydrogenase [Burkholderia cepacia complex]

MULTSPECLS: aldehyde dehydrogenase [Burkholderia cepacia complex]

MULTIISPECIES: aldehyde dehydrogenase [Burkholderia cepacia complex]

Chain A, The Crystal Structure Of Gamma-glutamyl-gamma-aminobutyraldehyde Dehydrogenase From Burkholderia Cenocepacia $J 2315$

MULTISPECESS

MULTISPECIES: methylmalonate-semialdehyde dehydrogenase (acylating) [Burkholderi cepcia conpex]

MULTISPECIES: acyl dehydratase [Burkholderia cepacia complex]

MULTISPECIES: acetyl-COA acetyltransferase [Burkholderia cepacia complex]

MULTISPECIES: acetyl-COA acetyltransferase [Burkholderia cepacia complex]

MULTISPECIES: acetyl-COA acetyltransferase [Burkholderia cepacia complex]

MULTISPECIIS: acetyl-CoA acetyytransferase [Burkholderia cepacia complex] MULTISPECIES: 3-ketoacyl-COA thiolase [Burkholderia]

MULTISPECIES: acety-COA acetyltransferase [Burkholderia cepacia complex]

MULTISPECIES acetyl-COA acetyltransferse [Burkholderia cep ria complex]

MULTISPECIES: acetyl-COA acetyltransferase [Burkholderia cepacia complex]

MULTSPECESS acety-COA acetyltransferase BBurkholderia cepacia complex]

MULTISPECIES: acetyl-CoA acetyltransferase [Burkholderia cepacia complex

MULTSPECIES: acetyl-CoA acetyltransferase Burkholderia cepacia complex MUISPCCES. acety-COA acetyltransferase [Burkholderia cepacia complex] MULTISPECIES: 3-ketoacyl-CoA thiolase [Burkholderia]

MULTISPECISS a MULTISPECIES: acetyl-COA acetyltransferase [Burkholderia cep cia conplex] MULTISPECIES: acetyl-COA acetyltransferase [Burkholderia cepacia complex] MULTISPECIES: acetyl-COA acetyltransferase [Burkholderia cepacia complex]
MULTISPECIES: acetyl-COA acetyltransferase [Burkholderia cepacia complex]
Scor

\begin{tabular}{|c|c|c|c|c|}
\hline .9 & 95.9 & $39 \%$ & $2.00 \mathrm{E}-21$ & \\
\hline 9.4 & 89.4 & $42 \%$ & 2.00E-19 & $28 \%$ WP_006484845.1 \\
\hline 87.8 & 87.8 & $52 \%$ & 7.00E-19 & 31\% WP_006489660.1 \\
\hline 87.8 & 87.8 & $52 \%$ & 7.00E-19 & $31 \%$ 4YWE_A \\
\hline 87.8 & 87.8 & $72 \%$ & $9.00 \mathrm{E}-19$ & 27\% 406R_A \\
\hline 87.4 & 87.4 & $72 \%$ & $1.00 \mathrm{E}-18$ & $27 \%$ WP_006484608.1 \\
\hline 87 & 87 & $52 \%$ & $1.00 E-18$ & $26 \%$ WP_006487205.1 \\
\hline 82.8 & 82.8 & $51 \%$ & $3.00 \mathrm{E}-17$ & $28 \%$ WP_034178280.1 \\
\hline 82.8 & 82.8 & $51 \%$ & $3.00 E-17$ & $28 \%$ CAR54273.1 \\
\hline 81.6 & 81.6 & $50 \%$ & $8.00 \mathrm{E}-17$ & 24\% WP_006490715.1 \\
\hline 79 & 79 & $52 \%$ & 5.00E-16 & $25 \%$ WP_006481882.1 \\
\hline 78.2 & 78.2 & $52 \%$ & 1.00E-15 & $28 \%$ WP_006484740.1 \\
\hline 78.2 & 78.2 & $52 \%$ & $1.00 \mathrm{E}-15$ & $28 \%$ CAR56157.1 \\
\hline 77.8 & 77.8 & $52 \%$ & $1.00 E-15$ & $28 \%$ 405H_A \\
\hline 73.2 & 73.2 & $51 \%$ & 4.00E-14 & 26\% WP_012493562.1 \\
\hline 70.5 & 70.5 & $42 \%$ & $2.00 E-13$ & 23\% WP_006491137.1 \\
\hline 70.5 & 70.5 & $42 \%$ & $3.00 E-13$ & $23 \%$ WP_006485777.1 \\
\hline 70.5 & 70.5 & $41 \%$ & 3.00E-13 & $26 \% 4 L{ }^{\prime}{ }_{-} \mathrm{A}$ \\
\hline 70.1 & 70.1 & $41 \%$ & $3.00 \mathrm{E}-13$ & 25\% WP_006490987.1 \\
\hline 70.5 & 70.5 & $41 \%$ & 3.00E-13 & 26\% WP_006488053.1 \\
\hline 69.7 & 69.7 & $42 \%$ & $5.00 \mathrm{E}-13$ & 26\% WP_012493582.1 \\
\hline 64.7 & 64.7 & $21 \%$ & $6.00 \mathrm{E}-13$ & $30 \%$ WP_006483300.1 \\
\hline 67.4 & 67.4 & $44 \%$ & 2.00E-12 & 25\% WP_006488924.1 \\
\hline 66.6 & 66.6 & $49 \%$ & $4.00 E-12$ & $26 \%$ WP_006481750.1 \\
\hline 60.1 & 60.1 & $52 \%$ & 5.00E-10 10 & 26\% WP_006489632.1 \\
\hline 100 & 100 & $52 \%$ & $5.00 \mathrm{E}-23$ & 26\% WP_006488133.1 \\
\hline 97.1 & 97.1 & $69 \%$ & $1.00 E-21$ & 24\% WP_012493582.1 \\
\hline 95.5 & 95.5 & $51 \%$ & $3.00 E-21$ & $28 \%$ WP_006487205.1 \\
\hline 87.8 & 87.8 & $51 \%$ & $9.00 \mathrm{E}-19$ & $26 \%$ WP_006481882.1 \\
\hline 87 & 87 & $56 \%$ & $1.00 \mathrm{E}-18$ & 24\% WP_006491137.1 \\
\hline 86.7 & 86.7 & $56 \%$ & 2.00E-18 & 24\% WP_006485777.1 \\
\hline 85.9 & 85.9 & $55 \%$ & $3.00 \mathrm{E}-18$ & 24\% WP_006493412.1 \\
\hline 85.9 & 85.9 & $55 \%$ & $3.00 E-18$ & $24 \%$ CAR5 $5358.1^{-1}$ \\
\hline 85.5 & 85.5 & $51 \%$ & $5.00 \mathrm{E}-18$ & 28\% WP_012493562.1 \\
\hline 81.3 & 81.3 & $37 \%$ & $9.00 \mathrm{E}-17$ & 26\% WP_006490987.1 \\
\hline 77.8 & 77.8 & $42 \%$ & $1.00 \mathrm{E}-15$ & $25 \%$ WP_006490715.1 \\
\hline 72.8 & 72.8 & $41 \%$ & 5.00E-14 & $25 \%$ WP_006484845.1 \\
\hline 70.9 & 70.9 & $40 \%$ & $2.00 \mathrm{E}-13$ & $25 \% 4$ 4LI__A \\
\hline 70.9 & 70.9 & $40 \%$ & $2.00 E-13$ & 25\% WP_006 \\
\hline 68.9 & 68.9 & $42 \%$ & $8.00 \mathrm{E}-13$ & 26\% WP_006488924.1 \\
\hline 62.8 & 62.8 & $19 \%$ & $3.00 E-12$ & $32 \%$ WP_006483300.1 \\
\hline 570 & 570 & $98 \%$ & 0 & 73\% WP_006491099.1 \\
\hline 552 & 552 & $98 \%$ & & $70 \%$ WP_006482586.1 \\
\hline 307 & 307 & $98 \%$ & $1.00 E-102$ & $46 \%$ WP_006485256.1 \\
\hline 307 & 307 & $98 \%$ & $2.00 E-102$ & $46 \%$ WP_012492402.1 \\
\hline 296 & 296 & $98 \%$ & $3.00 E-98$ & $46 \%$ WP_006485733.1 \\
\hline 287 & 287 & $98 \%$ & $9.00 \mathrm{E}-95$ & 44\% WP_006481441.1 \\
\hline 270 & 270 & $97 \%$ & $2.00 E-88$ & 43\% WP_006493096.1 \\
\hline 254 & 254 & $98 \%$ & $9.00 E-82$ & 42\% WP_006483911.1 \\
\hline 249 & 249 & $97 \%$ & $6.00 \mathrm{E}-80$ & $42 \%$ WP_006493100.1 \\
\hline 242 & 242 & $97 \%$ & $5.00 E-77$ & 39\% WP_006491472.1 \\
\hline 541 & 541 & $100 \%$ & 0 & 69\% WP_006482586.1 \\
\hline 535 & 535 & $100 \%$ & 0 & $69 \%$ WP_006491099.1 \\
\hline 323 & 323 & $100 \%$ & 6.00E-109 & $46 \% \mathrm{~W}$ \\
\hline 321 & 321 & $99 \%$ & $4.00 E-108$ & $46 \% \mathrm{WP}_{-}$ \\
\hline 310 & 310 & $99 \%$ & 8.00 & 45 \\
\hline 299 & 299 & $100 \%$ & $2.00 E-99$ & 44\% WP_006485733.1 \\
\hline 265 & 265 & $100 \%$ & $2.00 \mathrm{E}-86$ & $40 \%$ WP_006493096.1 \\
\hline 262 & 262 & $99 \%$ & $4.00 \mathrm{E}-85$ & 42\% WP_006483911.1 \\
\hline 252 & 252 & $99 \%$ & $6.00 \mathrm{E}-81$ & \\
\hline 229 & 229 & $98 \%$ & $4.00 E-72$ & 37\% WP_0064914 \\
\hline
\end{tabular}




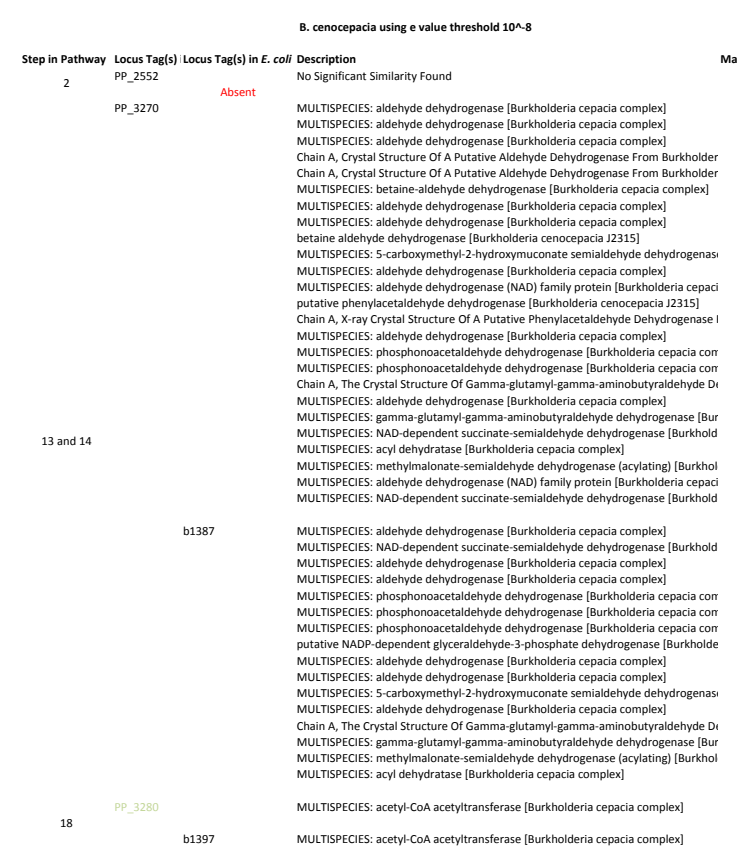

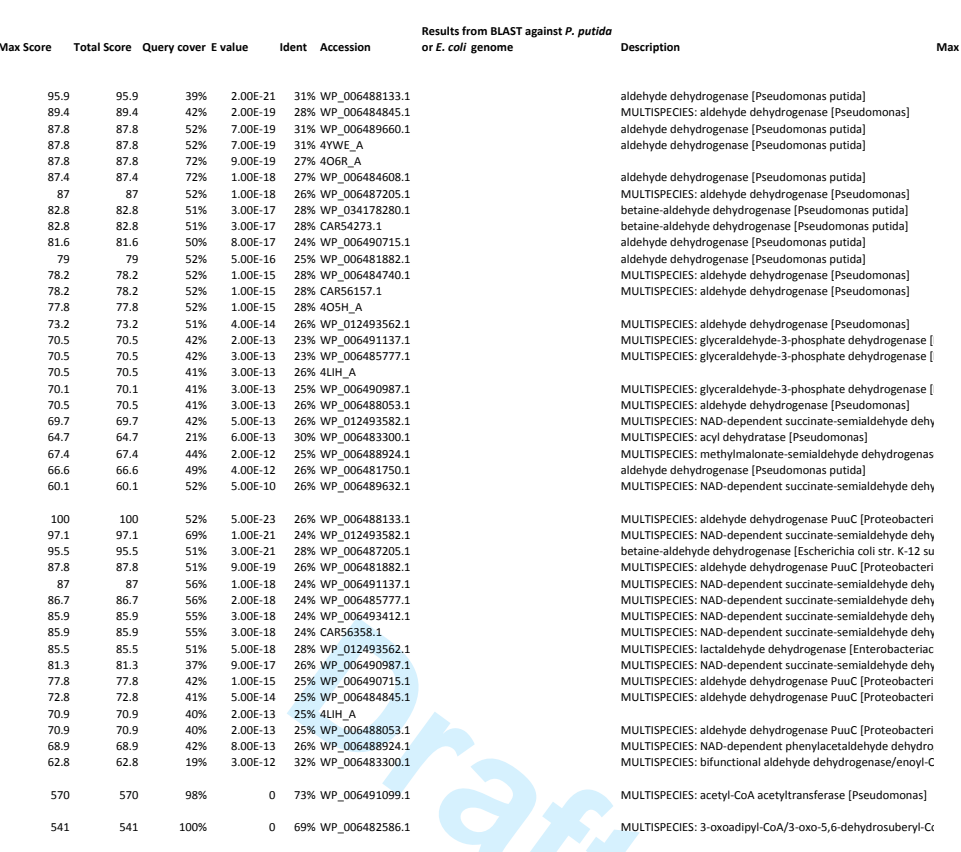

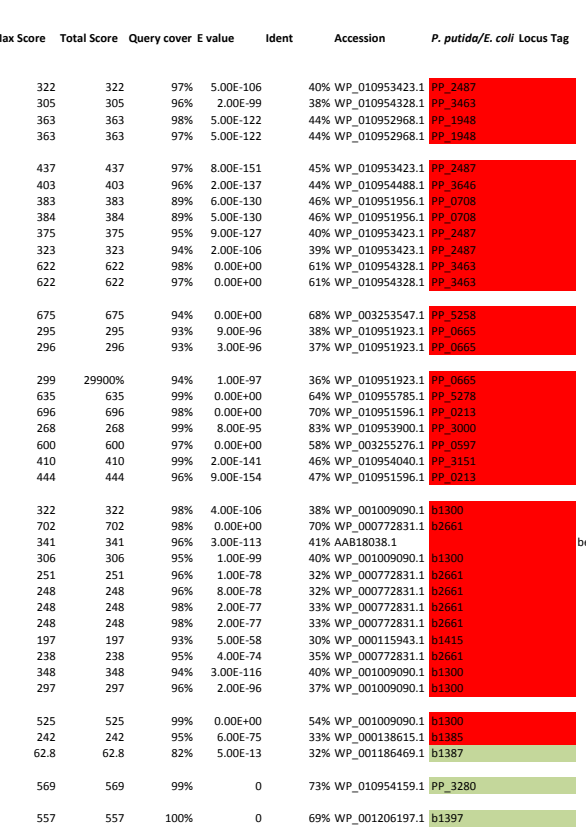

BCA50364 Bcalo407
всам2568 


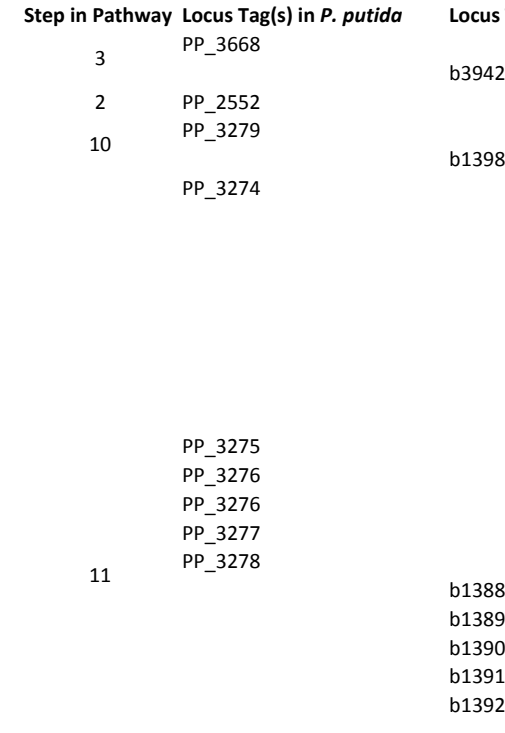

PP_3270 b1398
Locus Tag(s) in E. coli b3942 Absent
P. aeruginosa using e value threshold $10^{\wedge}-8$

Description

Max Score Total Score Query cover Evalue

No Significant Similarity Found

No Significant Similarity Found

No Significant Similarity Found

No Significant Similarity Found

MULTISPECIES: hybrid-cluster NAD(P)-dependent oxidoreductase [Pseudomonas]

MULTISPECIES: flarohemoprotein [Pseudomonas]

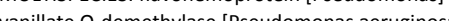

oxidoreductase [Pseudomonas aeruginosa]

oxidoreductase [Pseudomonas aeruginosa
ferredoxin [Pseudomonas aeruginosa]

Vanillate O-demethylase oxidoreductase [Pseudomonas aeruginosa]

MULTISPECIES: toluate 1,2-dioxygenase electron transfer subunit [Pseudomonas]

anthranilate dioxygenase reductase [Pseudomonas aeruginosa]

anthranilate dioxygenase reductase [Pseudomonas aeruginosa]

No Significant Similarity Found

No Significant Similarity Found

No Significant Similarity Found

No Significant Similarity Found

No Significant Similarity Found

No Significant Similarity Found

No Significant Similarity Foun

No Significant Similarity Found

MULTISPECIES: hybrid-cluster NAD(P)-dependent oxidoreductase [Pseudomonas]

MULTISPECIES: flavohemoprotein [Pseudomonas]

oxidoreductase [Pseudomonas aeruginos

ferredoxin [Pseudomonas aeruginosa]

vanillate $\mathrm{O}$-demethylase [Pseudomonas aeruginosa]

Vanillate O-demethylase oxidoreductase [Pseudomonas aeruginosa]

MULTISPECIES: oxidoreductase [Pseudomonas]

anthranilate dioxygenase reductase [Pseudomonas aeruginosa]
anthranilate dioxygenase reductase [Pseudomonas aeruginosa]

MULTISPECIES: toluate 1,2-dioxygenase electron transfer subunit [Pseudomonas]

aldehyde dehydrogenase [Pseudomonas aeruginosa]

MULTISPECIES: aldehyde dehydrogenase [Pseudomonas]

MULTISPECIES: betaine-aldehyde dehydrogenase [Pseudomonas]

betaine-aldehyde dehydrogenase [Pseudomonas aeruginosa]

MULTISPECIES: aldehyde dehydrogenase [Pseudomonas]

aldehyde dehydrogenase [Pseudomonas aeruginosa]

MULTISPECIES: aldehyde dehydrogenase [Pseudomonas]

MULTISPECIES: NAD-dependent succinate-semialdehyde dehydrogenase [Pseudomonas]

MULTISPECIES: aldehyde dehydrogenase [Pseudomonas]

aldehyde dehydrogenase [Pseudomonas aeruginosa]

putative aldehyde dehydrogenase [Pseudomonas aeruginosa PAO1H2O]

aldehyde dehydrogenase [Pseudomonas aeruginosa]

aldehyde dehydrogenase [Pseudomonas aeruginosa]

MULTISPECIES: aldehyde dehydrogenase [Pseudomonas]

aldehyde dehydrogenase [Pseudomonas aeruginosa]

MULTSPCL: 5-cas

[5endomonas]

dPseudomonas aeruginosa]

MULTISPECIES. hypothetical protein [Pseudomonas]

$\begin{array}{rrrrl}143 & 143 & 98 \% & 7.00 E-40 & 29 \% \text { WP_003096769.1 } \\ 93.2 & 93.2 & 59 \% & 1.00 E-21 & 30 \% \text { WP_003113387.1 } \\ 88.6 & 88.6 & 84 \% & 3.00 E-20 & 29 \% \text { WP_003112295.1 } \\ 82.8 & 82.8 & 79 \% & 4.00 E-18 & 27 \% \text { WP_003112305.1 } \\ 81.6 & 81.6 & 79 \% & 1.00 E-17 & 27 \% \text { WP_038404013.1 } \\ 79 & 79 & 84 \% & 6.00 E-17 & 29 \% \text { WP_038404026.1 } \\ 67.8 & 67.8 & 59 \% & 4.00 E-13 & 26 \% \text { WP_003109537.1 } \\ 61.6 & 61.6 & 68 \% & 2.00 E-11 & 25 \% \text { WP_047696755.1 } \\ 62 & 62 & 68 \% & 4.00 E-11 & \text { 25\% WP_003113312. }\end{array}$

\begin{tabular}{|c|c|c|}
\hline & $1.00 \mathrm{E}$ & $26 \% \mathrm{~W}$ \\
\hline & & 0\% WP_0031133 \\
\hline & 10E-19 & 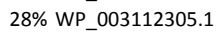 \\
\hline & & \\
\hline & OE-14 & 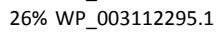 \\
\hline & & \\
\hline & $1.00 E-12$ & \% WP_003112912. \\
\hline & 12 & \\
\hline & $2.00 E-12$ & \% WP_003 \\
\hline & 10 & 37.1 \\
\hline & & 6.1 \\
\hline & $0 E-21$ & $1111 / 13.1$ \\
\hline & OE-21 & $\%$ \\
\hline & OE-20 & 436 \\
\hline & 19 & 1.1 \\
\hline & OE-17 & 2149.1 \\
\hline & & \\
\hline$\%$ & 15 & 3112906.1 \\
\hline & 5 & 3106269. \\
\hline $3 \%$ & 00E-13 & \% WP_003107137.1 \\
\hline & 00E-13 & $\%$ WP_038404021. \\
\hline$\%$ & OE-13 & 383.1 \\
\hline & ODE-13 & 17707 \\
\hline$\%$ & 13 & 8403762.1 \\
\hline & & \\
\hline & 3 & /P_038403149.1 \\
\hline & & \\
\hline & & \\
\hline & & \\
\hline & & \\
\hline & & \\
\hline
\end{tabular}


aldehyde dehydrogenase [Pseudomonas aeruginosa] MULTISPECIES: aldehyde dehydrogenase [Pseudomonas] MULTISPECIES: aldehyde dehydrogenase [Pseudomon

NADP-dendrencente-phosphag

enase [Pseudomonas aeruginosa]

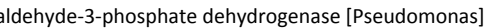

b1387

NADP-dependent glyceraldehyde-3-phosphate dehydrogenase [Pseudomonas aeruginosa]

MULTISPECIES: NAD-dependent succinate-semialdehyde dehydrogenase [Pseudomonas] aldehyde dehydrogenase [Pseudomonas aeruginosa]

MULTISPECIES: betaine-aldehyde dehydrogenase [Pseudomona

betaine-aldehyde dehydrogenase [Pseudomonas aeruginosa]

MULTISPECIES: aldehyde dehydrogenase [Pseudomonas]

aldehyde dehydrogenase [Pseudomonas aeruginosa]

TULTSPEILS. 5 -carboxy-2

5-carboxymethyl-2-hydroxymuconate semialdehyde dehydrogenase [Pseudomonas aeruginosa]

MULTISPECIES: hypothetical protein [Pseudomonas]

MUTTSPCCS: hypouhetical protein [Pseudomonas]

MULTISPECIES: beta-ketoadipyI CoA thiolase [Pseudomonas]

MULTISPECIES: acetyl-CoA acetyltransferase [Pseudomon

beta-ketoadipyl CoA thiolase [Pseudomonas aeruginosa]

MULTISPECIES: acetyl-COA acetyltransferase [Pseudomonas]

acetyl-CoA acetyltransferase [Pseudomonas aeruginos]

3-ketoacyl-CoA thiolase [Pseudomonas aeruginosa]

MULTISPECIES: 3-ketoacyl-CoA thiolase [Pseudomonas]

MULTISPECIES: acetyl-CoA acetyltransferase [Pseudomonas]

MULTISPECIES: acetyl-CoA acetyltransferase [Pseudomonas]

MULTISPECIES: acyl-CoA thiolase [Pseudomonas]

MULTISPECIES: acYl-CoA thiolase [Pseudomonas]

acetyl-CoA acetyltransferase [Pseudomonas aeruginosa]

MULTISPECIES: acyl-COA thiolase [Pseudomonas]

acetyl-CoA acetyltransferase [Pseudomonas aeruginosa]

MULTISPECIES: acyl-CoA thiolase [Pseudomonas]

acetyl-CoA acetyltransferase [Pseudomonas aeruginosa]

acetyl-CoA acetyltransferase [Pseudomonas aeruginosa]

acetyl-COA acetyltransferase [Pseudomonas aeruginosa]

acetyl-CoA acetyltransferase [Pseudomonas aeruginosa]

MULTISPECIES: acetyl-CoA acetyltransferase [Pseudomonas]

putative acetyl-CoA acetyltransferase [Pseudomonas aeruginosa MPAO1/P1]

MULTISPECIES: beta-ketoadipyl CoA thiolase [Pseudomonas]

MULTISPECIES: acetyl-CoA acetyltransferase [Pseudomonas]

beta-ketoadipyl CoA thiolase [Pseudomonas aeruginosa]

MULTISPECIES: acetyl-CoA acetyltransferase [Pseudomonas]

acetyl-CoA acetyltransferase [Pseudomonas aeruginosa]

3-ketoacyl-CoA thiolase [Pseudomonas aeruginosa]

MULTISPECIES: 3-ketoacyl-CoA thiolase [Pseudomonas]

MULTISPECIES: acetyl-CoA acetyltransferase [Pseudomonas]

MULTISPECIES: acetyl-CoA acetyltransferase [Pseudomonas]

MULTISPECIES: acYl-CoA thiolase [Pseudomonas]

MULTISPECIES: acyl-CoA thiolase [Pseudomonas]

MULTISPECIES: acyl-COA thiolase [Pseudomonas]

acetyl-CoA acetyltransferase [Pseudomonas aeruginosa]

acetyl-CoA acetyltransferase [Pseudomonas aeruginos]

MULTISPECIES: acyl-COA thiolase [Pseudomonas]

acetyl-CoA acetyltransferase [Pseudomonas aeruginosa]

\begin{tabular}{|c|c|c|c|c|}
\hline 65.9 & 65.9 & $42 \%$ & $1.00 \mathrm{E}-11$ & 27\% WP_010895535.1 \\
\hline 65.5 & 65.5 & $51 \%$ & $1.00 \mathrm{E}-11$ & 26\% WP_003086325.1 \\
\hline 62 & 62 & $52 \%$ & $2.00 \mathrm{E}-10$ & 26\% WP_003113043.1 \\
\hline 61.6 & 61.6 & $52 \%$ & $2.00 \mathrm{E}-10$ & 26\% WP_038403780.1 \\
\hline 59.7 & 59.7 & $45 \%$ & $9.00 \mathrm{E}-10$ & 23\% WP_047705006.1 \\
\hline 60.1 & 60.1 & $45 \%$ & $9.00 \mathrm{E}-10$ & 23\% WP_003115067.1 \\
\hline 59.7 & 59.7 & $45 \%$ & $1.00 \mathrm{E}-09$ & $23 \%$ WP 038403238.1 \\
\hline 97.4 & 97.4 & $65 \%$ & $1.00 \mathrm{E}-21$ & 27\% WP_003107137.1 \\
\hline 95.1 & 95.1 & $65 \%$ & $5.00 \mathrm{E}-21$ & 24\% WP_003106269.1 \\
\hline 94.7 & 94.7 & $65 \%$ & $9.00 \mathrm{E}-21$ & 26\% WP_038404021.1 \\
\hline 91.3 & 91.3 & $67 \%$ & $1.00 \mathrm{E}-19$ & 27\% WP_003114436.1 \\
\hline 90.1 & 90.1 & $67 \%$ & $2.00 \mathrm{E}-19$ & 27\% WP_038404164.1 \\
\hline 88.2 & 88.2 & $42 \%$ & $1.00 \mathrm{E}-18$ & 28\% WP_003112906.1 \\
\hline 87.8 & 87.8 & $45 \%$ & $1.00 \mathrm{E}-18$ & 27\% WP_047697654.1 \\
\hline 78.6 & 78.6 & $42 \%$ & $1.00 \mathrm{E}-15$ & 27\% WP_003110130.1 \\
\hline 78.6 & 78.6 & $42 \%$ & $1.00 E-15$ & 27\% WP_003115626.1 \\
\hline 57.8 & 57.8 & $16 \%$ & $3.00 \mathrm{E}-10$ & 32\% WP_003091647.1 \\
\hline 57.4 & 57.4 & $12 \%$ & $4.00 E-10$ & 35\% WP_003093220.1 \\
\hline 541 & 541 & $98 \%$ & 0 & 68\% WP_003101891.1 \\
\hline 387 & 387 & $98 \%$ & $2.00 \mathrm{E}-133$ & $56 \%$ WP_0030 \\
\hline 384 & 384 & $98 \%$ & $3.00 E-132$ & 56\% WP_038403640.1 \\
\hline 324 & 324 & $98 \%$ & $6.00 E-109$ & 48\% WP_003121716.1 \\
\hline 323 & 323 & $98 \%$ & $1.00 E-108$ & 48\% WP_003113500.1 \\
\hline 287 & 287 & $98 \%$ & 2.00E-94 & 41\% WP_003115356.1 \\
\hline 286 & 286 & $98 \%$ & 4.00E-94 & 41\% WP_003113978.1 \\
\hline 278 & 278 & $98 \%$ & $5.00 E-91$ & 43\% WP_003087747.1 \\
\hline 276 & 276 & $93 \%$ & $3.00 E-90$ & 45\% WP_003101465.1 \\
\hline 275 & 275 & $93 \%$ & 4.00E-90 & 45\% WP_003114002.1 \\
\hline 244 & 244 & $97 \%$ & $1.00 E-77$ & 40\% WP_003114496.1 \\
\hline 234 & 234 & $98 \%$ & $9.00 E-74$ & 39\% WP_038403740.1 \\
\hline 234 & 234 & $98 \%$ & $1.00 E-73$ & 39\% WP_003103022.1 \\
\hline 233 & 233 & $98 \%$ & $1.00 \mathrm{E}-73$ & 39\% WP_003114307.1 \\
\hline 220 & 220 & $97 \%$ & $2.00 \mathrm{E}-68$ & 38\% WP_003108823.1 \\
\hline 210 & 210 & $78 \%$ & $1.00 E-65$ & 40\% WP_047697289.1 \\
\hline 203 & 203 & $85 \%$ & $2.00 \mathrm{E}-62$ & 38\% WP_003115775.1 \\
\hline 200 & 200 & $80 \%$ & $1.00 E-61$ & 39\% WP_047705128.1 \\
\hline 196 & 196 & $78 \%$ & $3.00 E-60$ & 38\% WP_047696772.1 \\
\hline 125 & 125 & $97 \%$ & $1.00 E-32$ & 29\% WP_003102247.1 \\
\hline 94.7 & 94.7 & $24 \%$ & $2.00 E-23$ & 53\% EHS33070.1 \\
\hline 531 & 531 & $99 \%$ & 0 & 65\% WP_003101891.1 \\
\hline 389 & 389 & $99 \%$ & $1.00 E-134$ & 54\% WP_003098466.1 \\
\hline 387 & 387 & $99 \%$ & $1.00 E-133$ & 53\% WP_038403640.1 \\
\hline 310 & 310 & $99 \%$ & $2.00 E-103$ & 46\% WP_003121716.1 \\
\hline 309 & 309 & $99 \%$ & $5.00 E-103$ & 46\% WP_003113500.1 \\
\hline 300 & 300 & $99 \%$ & $1.00 E-99$ & 42\% WP_003115356.1 \\
\hline 300 & 300 & $99 \%$ & 2.00E-99 & 42\% WP_003113978.1 \\
\hline 284 & 284 & $100 \%$ & 4.00E-93 & 42\% WP_003087747.1 \\
\hline 282 & 282 & $95 \%$ & 7.00E-93 & 45\% WP_003101465.1 \\
\hline 282 & 282 & $95 \%$ & $8.00 E-93$ & 45\% WP_003114002.1 \\
\hline 254 & 254 & $100 \%$ & $9.00 E-82$ & 40\% WP_003114496.1 \\
\hline 231 & 231 & $99 \%$ & $6.00 E-73$ & 39\% WP_003103022.1 \\
\hline 231 & 231 & $99 \%$ & $7.00 E-73$ & 39\% WP_003114307.1 \\
\hline 231 & 231 & $99 \%$ & $7.00 E-73$ & 39\% WP_038403740.1 \\
\hline 201 & 201 & $78 \%$ & 4.00E-62 & 39\% WP_047697289.1 \\
\hline 201 & 201 & $98 \%$ & $3.00 E-61$ & 36\% WP_003108823.1 \\
\hline 186 & 186 & $86 \%$ & 3.00E-56 & 36\% WP_003115775.1 \\
\hline
\end{tabular}



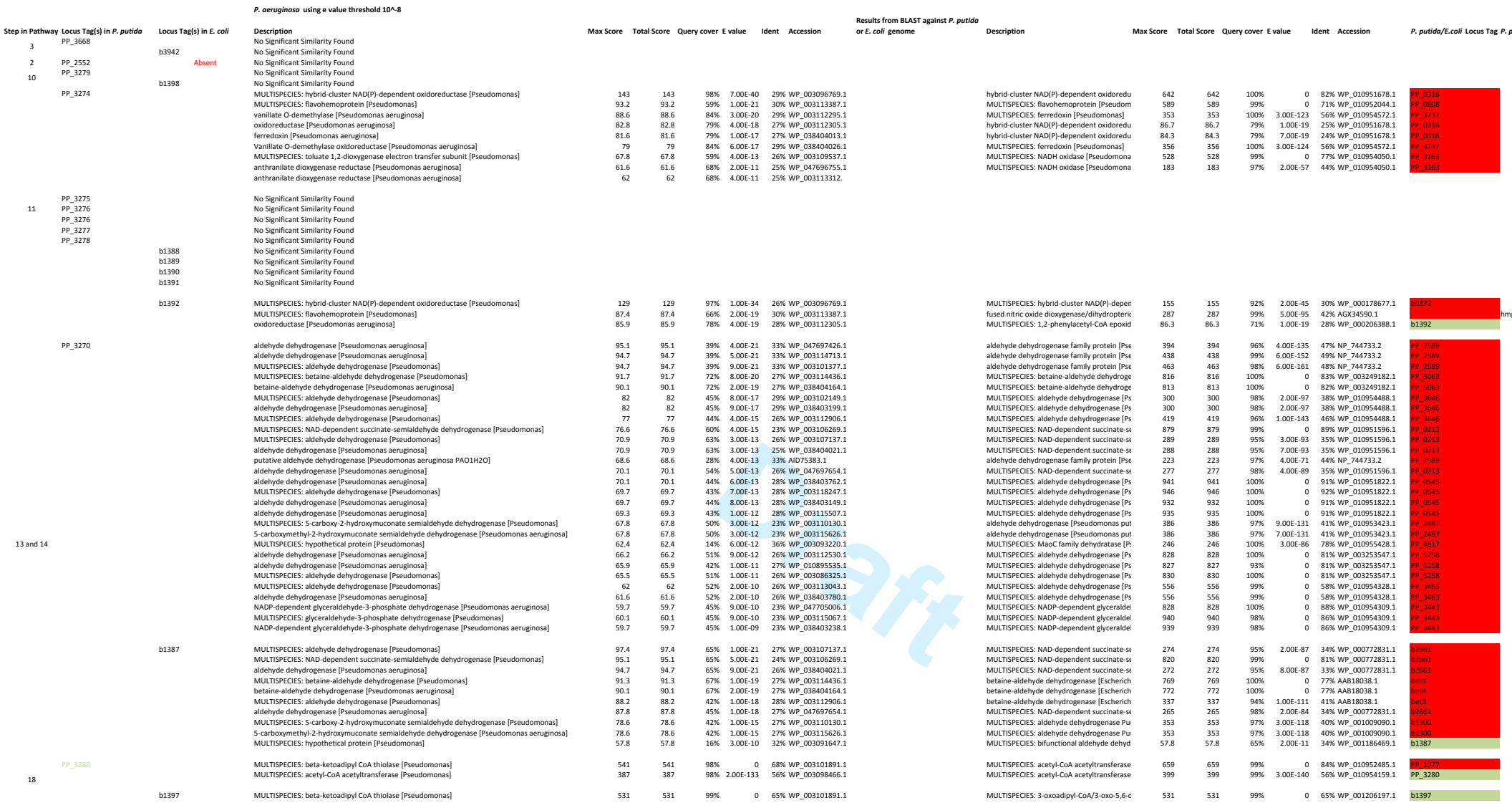
Step in Pathway Locus Tag(s) in P. putida Locus Tag(s) in E. coli PP_3463

b1385

\section{Description}

aldehyde dehydrogenase AldA [Aspergilius fumigatus Af293] aldehyde dehydrogenase ALDH [Aspergillus fumigatus Af293] aldehyde dehydrogenase [Aspergillus fumigatus Af293] aldehyde dehydrogenase [Aspergillus fumigatus Af293] aldehyde dehydrogenase [Aspergillus fumigatus A2293] betaine aldehyde dehydrogenase (BadH) [Aspergillus fumigatus Af293] succinate-semialdehyde dehydrogenase [Aspergillus fumigatus Af29 betaine aldehyde dehydrogenase [Aspergillus fumigatus Af293] succinate-semialdehyde dehydrogenase Uga2 [Aspergillus fumigatus Af293] succinate-semialdehyde dehydrogenase [Aspergillus fumigatus Af29 (a) aldehyde dehydrogenase family protein [Aspergillus fumigatus Af293] aldehyde dehydrogenase family protein [Aspergillus fumigatus A A293] aldehyde dehydrogenase family protein [Aspergillus fumigatus A2293] aldehyde dehydrogenase family protein [Aspergillus fumigatus Af293] methylmalonate-semialdehyde dehydrogenase [Aspergillus fumigatus Af293] vanillin dehydrogenase [Aspergillus fumigatus A A293]

delta-1-pyrroline-5-carboxylate dehydrogenase PrnC [Aspergillus fumigatus Af293] aldehyde dehydrogenase [Aspergillus fumigatus Af293] aldehyde dehydrogenase [Aspergillus fumigatus Af293]

aldehyde dehydrogenase [Aspergillus fumigatus A 2933$]$ aldehyde dehydrogenase [Asperglilus fumigatus Af293] aldehyde dehydrogenase AldA [Aspergllus fumigatus Af293] aldehyde dehyrogerse [Asperillus fumigotus A 2931 ? aldehyde dehydrogenase [Aspergillus fumigatus Af29 betaine aldehyde dehydrogenase (BadH) [Aspergillus fumigatus Af293] betaine aldehyde dehydrogenase [Aspergillus fumigatus Af293] aldehyde dehydrogenase (AldH12) [Aspergillus fumigatus Af293] aldehyde dehydrogenase family protein [Aspergillus fumigatus Af293] succinate-semialdehyde dehydrogenase [Aspergillus fumigatus A A293] succinate-semialdehyde dehydrogenase Uga2 [Aspergillus fumigatus Af293] succinate-semialdehyde dehydrogenase [Aspergillus fumigatus Af293] aldehyde dehydrogenase family protein [Aspergillus fumigatus At29] aldehyde dehydrogenase family protein [Aspergillus fumigatus A2293] aldehyde dehydrogenase family protein [Aspergllus fumigatus A2293] vanilin dehydrogenase [Aspergillus fumigatus A $\mathrm{A293}$ ] (Aspergillus fumigatus Af293] aldehyde dehydrogenase [Asperillus fumigatus Af293] delta-1-pyrroline-5-carboxylate dehydrogenase Prnc [Aspergillus fumigatus Af293] aldehyde dehydrogenase [Aspergillus fumigatus Af293]

No Significant Similarity Found

No Significant Similarity Found

No Significant Similarity Found

cytochrome b5 reductase [Aspergillus fumigatus Af293] flavohemoprotein [Aspergillus fumigatus Af293]

No Significant Similarity Found

No Significant Similarity Found

No Significant Similarity Found

No Significant Similarity Found

\begin{tabular}{|c|c|c|c|c|}
\hline x score & Total Score & Query cover & Evalue & Ident Accession \\
\hline 420 & 420 & $96 \%$ & 8.00E-143 & 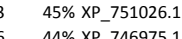 \\
\hline 404 & 404 & $95 \%$ & $3.00 E-136$ & $44 \%$ XP_746975.1 \\
\hline 400 & 400 & $98 \%$ & $5.00 E-135$ & 42\% XP_751967.1 \\
\hline 396 & 396 & $96 \%$ & $2.00 E-134$ & $43 \%$ XP_746831.1 \\
\hline 363 & 363 & $94 \%$ & 2.00E- -121 & $43 \%$ ХР_749209.1 \\
\hline 282 & 282 & $91 \%$ & $4.00 E-90$ & $36 \%$ XP_746469.1 \\
\hline 283 & 283 & $94 \%$ & $4.00 E-90$ & $38 \%$ XP_747215.1 \\
\hline 281 & 281 & $94 \%$ & $1.00 \mathrm{E}-89$ & $37 \%$ XP_748332.1 \\
\hline 278 & 278 & $94 \%$ & $2.00 \mathrm{E}-88$ & 37\% XP_749799.1 \\
\hline 270 & 270 & $98 \%$ & $4.00 \mathrm{E}-85$ & $35 \%$ XP_752009.1 \\
\hline 266 & 266 & $94 \%$ & $8.00 \mathrm{E}-84$ & 34\% XP_754891.1 \\
\hline 265 & 265 & $93 \%$ & $2.00 \mathrm{E}-83$ & $35 \%$ XP_756081.1 \\
\hline 253 & 253 & $91 \%$ & $1.00 \mathrm{E}-78$ & 34\% XP_748644.1 \\
\hline 244 & 244 & $90 \%$ & $2.00 E-75$ & 34\% XP_746990.1 \\
\hline 216 & 216 & $92 \%$ & $7.00 \mathrm{E}-65$ & 33\% XP_747354.1 \\
\hline 213 & 213 & $93 \%$ & $7.00 \mathrm{E}-64$ & 31\% XP_746556.2 \\
\hline 212 & 212 & $93 \%$ & $2.00 \mathrm{E}-63$ & 30\% XP_755901.1 \\
\hline 201 & 201 & $97 \%$ & $3.00 E-58$ & $29 \% \mathrm{XP}_{-}^{-} 751545.1$ \\
\hline 184 & 184 & $91 \%$ & $6.00 \mathrm{E}-53$ & $32 \% \mathrm{XP}_{-}^{-} 750112.1$ \\
\hline 180 & 180 & $96 \%$ & $4.00 E-50$ & $27 \% \mathrm{XP}_{-}^{-} 747700.1$ \\
\hline 133 & 133 & $93 \%$ & $8.00 E-34$ & $27 \%$ XP_750764.1 \\
\hline 130 & 130 & $84 \%$ & $2.00 \mathrm{E}-33$ & $28 \%$ XP_746302.1 \\
\hline 127 & 127 & $88 \%$ & $6.00 \mathrm{E}-32$ & $26 \%$ XP_751481.1 \\
\hline 367 & 367 & $95 \%$ & $6.00 \mathrm{E}-122$ & 40\% XP_751967.1 \\
\hline 358 & 358 & $95 \%$ & $1.00 E-119$ & $39 \% \mathrm{XP}_{-} 746831.1$ \\
\hline 348 & 348 & $98 \%$ & $1.00 E-114$ & 37\% XP_751026.1 \\
\hline 335 & 335 & $95 \%$ & $2.00 E-109$ & $40 \%$ XP_746975.1 \\
\hline 331 & 331 & $95 \%$ & $6.00 E-109$ & $9 \% \times$ XP_7 $_{7} 79209.1$ \\
\hline 280 & 280 & $93 \%$ & $3.00 E-89$ & $36 \% \mathrm{XP}_{-}^{-} 746469.1$ \\
\hline 280 & 280 & $95 \%$ & $7.00 \mathrm{E}-89$ & $36 \% \times \mathrm{XP}_{-} 747215.1$ \\
\hline 277 & 277 & $95 \%$ & $6.00 \mathrm{E}-88$ & $36 \%$ XP_749799.1 \\
\hline 276 & 276 & $94 \%$ & $1.00 \mathrm{E}-87$ & 36\% XP_756081.1 \\
\hline 276 & 276 & $95 \%$ & $2.00 \mathrm{E}-87$ & $35 \%$ XP_748644.1 \\
\hline 266 & 266 & $90 \%$ & $1.00 E-83$ & $34 \%$ XP_$_{-}^{-} 754891.1$ \\
\hline 254 & 254 & $90 \%$ & $4.00 E-79$ & $33 \% \mathrm{XP}_{-}^{-} 746990.1$ \\
\hline 254 & 254 & $94 \%$ & $6.00 \mathrm{E}-79$ & 35\% XP_752009.1 \\
\hline 252 & 252 & $94 \%$ & $2.00 E-78$ & $33 \%$ XP_748332.1 \\
\hline 242 & 242 & $91 \%$ & $8.00 \mathrm{E}-75$ & $35 \%$ XP_747354.1 \\
\hline 221 & 21 & & $6.00 \mathrm{E}-67$ & $29 \% \mathrm{XP}_{-}^{-} 746556.2$ \\
\hline 217 & & & $2.00 \mathrm{E}-65$ & $29 \%$ XP_755901.1 \\
\hline 214 & & $92 \%$ & $4.00 E-64$ & $35 \%$ XP_750112.1 \\
\hline 201 & 201 & $94 \%$ & $4.00 E-58$ & $30 \% \mathrm{XP}_{-}^{-} 751545.1$ \\
\hline 183 & 183 & $93 \%$ & $2.00 \mathrm{E}-51$ & $30 \%$ XP_747700.1 \\
\hline 160 & 160 & $84 \%$ & $5.00 \mathrm{E}-44$ & $30 \%$ XP_746302.1 \\
\hline 143 & 143 & $88 \%$ & $2.00 \mathrm{E}-37$ & $28 \%$ XP_750764.1 \\
\hline 141 & 141 & $67 \%$ & $5.00 \mathrm{E}-37$ & O\% XP_751481.1 \\
\hline
\end{tabular}

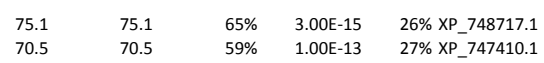


No Significant Similarity Found

No Significant Similarity Found

NADH-cyto b5 reduclase [Aspergillus funigatus Af293]

noyl-CoA hydratase/isomerase family protein [Aspergillus fumigatus Af293] [Aspergillus fumigatus Af293]

[Aspergillus fumigatus Af293] enoyl-CoA hydratase/isomerase family protein [Aspergillus fumigatus Af293] mitochondrial methylglutaconyl-CoA hydratase (Auh) [Aspergillus fumigatus Af293] enoyl-CoA hydratase/isomerase family protein [Aspergillus fumigatus Af293]

enoyl-CoA hydratase/isomerase family protein [Aspergillus fumigatus Af293] enoyl-CoA hydratase/isomerase family protein [Aspergillus fumigatus Af293] enoyl-CoA hydratase/isomerase family protein [Aspergillus fumigatus Af293] enoyl-CoA hydratase/isomerase family protein [Aspergillus fumigatus Af293] mitochondrial methylglutaconyl-CoA hydratase (Auh) (Aspergillus fumigatus Af29] enoyl-CoA hydratase/isomerase family protein [Aspergillus fumigatus Af293] enoyl-CoA hydratase/isomerase family protein [Aspergillus fumigatus Af293]

Aspergillus fumigatus Af293]

enoyl-CoA hydratase/isomerase family protein [Aspergillus fumigatus Af293]

enoyl-CoA hydratase [Aspergillus fumigatus Af293]
enoyl-CoA hydratase/isomerase family protein [Aspergillus fumigatus Af293] enoyl-CoA hydratase/somerase family protein (Aspergillus fumigatus Af293 enoyl-COA hydrato/isomera family protein [Aspergillus fumigotus Af293] peroxisomal D3,D2-enoyl-CoA isomerase [Aspergillus fumigatus Af293] mitochondrial 3-hydroxyisobutyryl-CoA hydrolase [Aspergillus fumigatus Af293] enoyl-CoA hydratase/isomerase family protein [Aspergillus fumigatus Af293]

aldehyde dehydrogenase family protein [Aspergillus fumigatus Af293] aldehyde dehydrogenase family protein [Aspergllus fumige

enoyl-CoA hydratase/isomerase family protein [Aspergillus fumigatus Af293] enoy-COA hydratase [Asperginus rumigatus Airgs]

enoyl-CoA hydratase/somerase family protein (Aspergillus fumigatus Af293) mitochondrial methylglutaconyl COA hydratase (Aub) [Aspergillus fumigatus Af29 mitochondrial 3-hydroxyisobutyryl-CoA hydrolase [Aspergillus fumigatus Af293] enoyl-CoA hydratase/isomerase family protein [Aspergillus fumigatus Af293]

enoyl-CoA hydratase/isomerase family protein [Aspergillus fumigatus Af293] enoyl-CoA hydratase/isomerase family protein [Aspergillus fumigatus Af293] enoyl-COA hydratase/isomerase family protein [Aspergillus fumigatus Af23] mitochondrial methylgutaconyl-CoA hydratase (Auh) [Aspergillus fumigatus Af29 enoyl-CoA hydratase/isomerase family protein [Aspergillus fumigatus Af293] enoyl-CoA hydratase/isomerase family protein [Aspergillus fumigatus Af293] enoyl-CoA hydratase [Aspergillus fumigatus A 1293 ]

mitochondrial 3-hydroxyisobutyryl-CoA hydrolase [Aspergillus fumigatus Af293] peroxisomal D3,D2-enoyl-CoA isomerase [Aspergillus fumigatus Af293]

enoyl-CoA hydratase/isomerase family protein [Aspergillus fumigatus Af293] enoyl-CoA hydratase [Aspergillus fumigatus Af293] enoyl-COA hydratase/isomerase family protein [Aspergillus fumigatus Af293]
enoy-COA hydratase/isomerase family protein [Aspergillus fumigatus A2293

\begin{tabular}{|c|c|c|c|c|}
\hline 70.1 & 70.1 & $55 \%$ & $1.00 E-13$ & 24\% XP_753636.1 \\
\hline 63.9 & 63.9 & $65 \%$ & 9.00E-12 & $24 \%$ XP_755738.2 \\
\hline 121 & 121 & $98 \%$ & $2.00 E-33$ & $29 \%$ XP_755425.1 \\
\hline 103 & 103 & $97 \%$ & $2.00 \mathrm{E}-26$ & $30 \%$ XP_755398.1 \\
\hline 92.4 & 92.4 & $93 \%$ & $1.00 E-22$ & $27 \%$ XP_747085.1 \\
\hline 92 & 92 & $98 \%$ & $2.00 \mathrm{E}-22$ & $28 \%$ XP_748661.1 \\
\hline 78.6 & 78.6 & $95 \%$ & $2.00 E-17$ & $26 \% \times$ \\
\hline 76.6 & 76.6 & $73 \%$ & $3.00 E-16$ & $28 \%$ XP_-753374.1 \\
\hline 67 & 67 & $77 \%$ & $2.00 \mathrm{E}-13$ & $29 \%$ XP_754855.1 \\
\hline 106 & 106 & $96 \%$ & $1.00 E-27$ & $31 \%$ ХР 755425.1 \\
\hline 105 & 105 & $92 \%$ & $1.00 E-27$ & $31 \%$ XP_748661.1 \\
\hline 102 & 102 & $91 \%$ & $2.00 E-26$ & 31\% XP_747085.1 \\
\hline 100 & 100 & $93 \%$ & $3.00 \mathrm{E}-25$ & $32 \%$ XP_749938.1 \\
\hline 95.9 & 95.9 & $94 \%$ & $1.00 E-23$ & $31 \%$ XP_754471.1 \\
\hline 89.7 & 89.7 & $93 \%$ & $2.00 E-21$ & $28 \% \mathrm{XP}_{-}^{-} 754174.1$ \\
\hline 88.6 & 88.6 & $69 \%$ & $4.00 E-21$ & $34 \%$ ХР 754855.1 \\
\hline 86.7 & 86.7 & $98 \%$ & $3.00 E-20$ & $28 \% \mathrm{XP}_{-}^{-} 755398.1$ \\
\hline 81.3 & 81.3 & $74 \%$ & $9.00 E-18$ & $31 \%$ XP_753374.1 \\
\hline 67 & 67 & $84 \%$ & $2.00 \mathrm{E}-13$ & $28 \% \mathrm{XP}_{-}^{-} 747673.1$ \\
\hline 125 & 125 & $99 \%$ & $1.00 E-34$ & $31 \%$ XP_755425.1 \\
\hline 117 & 117 & $97 \%$ & $5.00 E-32$ & $31 \%$ XP_755398.1 \\
\hline 112 & 112 & $96 \%$ & $3.00 E-30$ & $32 \%$ XP_747085.1 \\
\hline 105 & 105 & $94 \%$ & $5.00 E-27$ & $31 \%$ XP_749938.1 \\
\hline 99.4 & 99.4 & $97 \%$ & $3.00 E-25$ & $29 \% \mathrm{XP}_{-}^{-} 748661.1$ \\
\hline 89.7. & 89.7 & $96 \%$ & $1.00 E-21$ & $26 \%$ ХР_754174.1 \\
\hline 89.4 & 89.4 & $89 \%$ & $2.00 E-21$ & $29 \% \times \mathrm{XP}^{-} 747673.1$ \\
\hline 80.9 & 80.9 & $71 \%$ & $1.00 E-17$ & $31 \% \mathrm{XP}_{-}^{-} 753374.1$ \\
\hline 67.8 & 67.8 & $78 \%$ & $1.00 \mathrm{E}-13$ & $28 \%$ XP_754855.1 \\
\hline 76.6 & 76.6 & $41 \%$ & $4.00 E-15$ & $25 \% \times$ \\
\hline 68.2 & 68.2 & $42 \%$ & $2.00 \mathrm{E}-12$ & $27 \%$ XP_755901.1 \\
\hline 62.8 & 62.8 & $66 \%$ & $1.00 E-10$ & $23 \%$ XP_751481.1 \\
\hline 83.2 & 83.2 & $65 \%$ & $5.00 \mathrm{E}-17$ & $25 \%$ XP_754891.1 \\
\hline 72.4 & 72.4 & $44 \%$ & $1.00 E-13$ & $25 \%$ XP_755901.1 \\
\hline 121 & 12: & $\%$ & 8.00 & $29 \%$ \\
\hline 103 & 103 & $97 \%$ & $2.00 \mathrm{E}-26$ & $30 \%$ XP_755398.1 \\
\hline 92.4 & 92.4 & $93 \%$ & $1.00 \mathrm{E}-22$ & $27 \%$ XP_747085.1 \\
\hline 92 & 92 & $98 \%$ & $2.00 E-22$ & $28 \%$ XP_748661.1 \\
\hline 78.6 & 78.6 & $95 \%$ & $2.00 E-17$ & $26 \%$ XP_754471.1 \\
\hline 76.6 & 76.6 & $73 \%$ & $3.00 \mathrm{E}-16$ & $28 \%$ XP_753374.1 \\
\hline 67 & 67 & $77 \%$ & 2.00E-13 & $29 \%$ XP_754855.1 \\
\hline 106 & 106 & $96 \%$ & & $31 \%$ \\
\hline 105 & 105 & $92 \%$ & $1.00 E-27$ & $31 \% \times P_{-} 748661.1$ \\
\hline 102 & 102 & $91 \%$ & $2.00 E-26$ & $31 \% \times P_{-} 747085.1$ \\
\hline 100 & 100 & $93 \%$ & $.00 \mathrm{E}-25$ & 32\% XP_749938.1 \\
\hline 95.9 & 95.9 & $94 \%$ & $1.00 E-23$ & $31 \%$ XP_754471.1 \\
\hline 89.7 & 89.7 & $93 \%$ & $2.00 \mathrm{E}-21$ & $28 \% \times \mathrm{XP}_{-} 754174.1$ \\
\hline 88.6 & 88.6 & $69 \%$ & DOE-21 & $34 \%$ XP_754855.1 \\
\hline 86.7 & 86.7 & $98 \%$ & & $28 \%$ XP_755398.1 \\
\hline 81.3 & 81.3 & $74 \%$ & $9.00 E-18$ & $31 \%$ XP_753374.1 \\
\hline 67 & 67 & $84 \%$ & 2.00E-13 & $28 \%$ XP_747673.1 \\
\hline 125 & 125 & $99 \%$ & $1.00 E-34$ & $31 \%$ XP_755425.1 \\
\hline 117 & 117 & $97 \%$ & $5.00 E-32$ & $31 \% \times P$ P 755398.1 \\
\hline 112 & 112 & $96 \%$ & 0 & $322^{\circ}>$ \\
\hline 105 & 105 & $\%$ & 27 & \\
\hline 99.4 & 99.4 & $97 \%$ & $3.00 \mathrm{E}-25$ & $29 \%$ XP_74866 \\
\hline
\end{tabular}


enoyl-CoA hydratase/isomerase family protein [Aspergillus fumigatus Af293] peroxisomal D3,D2-enoyl-CoA isomerase [Aspergillus fumigatus Af293] mitochondrial 3-hydroxyisobutyryl-CoA hydrolase [Aspergillus fumigatus Af2933
enoyl-CoA hydratase/isomerase family protein [Aspergillus fumigatus A2293]

\section{3-ketoacyl-CoA thiolase (POT1) [Aspergillus fumigatus Af293]} acetyl-CoA-acetyltransferase [Aspergillus fumigatus Af293] acety-COA-acetyltransferase [Aspergillus fumigatus At293]
peroxisomal 3-ketoacyl-coA thiolase (Kat1) [Aspergillus fumigatus Af293] 3-ketoacyl-coA thiolase peroxisomal A precursor [Aspergillus fumigatus Af293] 3-ketoacyl-CoA ketothiolase (Kat1) [Aspergillus fumigatus Af293] acetyl-CoA acetyltransferase [Aspergillus fumigatus Af293]

PP 1845

PP_2217

PP_3284

\section{No Significant Similarity Found}

enoyl-CoA hydratase/isomerase family protein [Aspergillus fumigatus Af293] enoyl-CoA hydratase/isomerase family protein [Aspergillus fumigatus Af293] enoy-CoA hydratase/isomerase family protein [AS
enoyl-CoA hydratase [Aspergillus fumigatus Af293]

enoy-CoA hydratase/isomerase family protein [Aspergillus fumigatus Af293] mitochondrial methylglutacony-CoA hydratase (Auh) (Aspergillus fumigatus A229 enoyl-CoA hydratase/isomerase family protein [Aspergillus fumigatus Af293] peroxisomal D3, D2 - enoy- CoA isomerase [Asperillus fumigatus Af293] mitochondrial 3-hydroxyisobutyryl-CoA hydrolase [Aspergillus fumigatus A2293]

enoyl-CoA hydratase/isomerase family protein [Aspergillus fumigatus Af293] enoyl-CoA hydratase/isomerase family protein [Aspergillus fumigatus Af293] enoyl-CoA hydratase (Aspergillus fumigatus Ai293)

enoyl-CoA hydratase//somerase family protein [Aspergillus fumigatus A2293] mitocho drial 3 hydroyysom enoyl-CoA hydratase/isomerase family protein [Aspergillus fumigatus Af293]. peroxisomal D3,D2-enoyl-CoA isomerase [Aspergillus fumigatus Af293]

enoyl-CoA hydratase/isomerase family protein [Aspergillus fumigatus Af293] enoyl-CoA hydratase/somerase family protein [Aspergillus fumigatus Af29] enoyl-CoA hydratase/somerse famly proten [Aspergllus fumigatus A293]

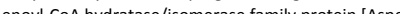
enoyl-CoA hydratase/isomerase family protein [Aspergillus fumigatus Af293] mitochondrial 3-hydroxyisobutyryl-CoA hydrolase [Aspergillus fumigatus Af293] peroxisomal D3,D2-enoyl-CoA isomerase [Aspergillus fumigatus Af293]

enoyl-CoA hydratase/isomerase family protein [Aspergillus fumigatus Af293] enoyl-CoA hydratase/somerase family protein (Aspergillus fumigatus A A293] enoyl-CoA hydratase [Aspergillus fumigatus Af293]

mitochondrial methylglutaconyl-CoA hydratase (Auh) [Aspergillus fumigatus Af293] enoyl-CoA hydratase/isomerase family protein [Aspergillus fumigatus Af293] enoyl-CoA hydratase/isomerase family protein [Aspergillus fumigatus Af29] enoyl-CoA hydratase/isomerase family protein [Aspergillus fumigatus Af293] mitochondrial 3-hydroxyisobutyryl-CoA hydrolase [Aspergillus fumigatus Af293]

3-ketoacy-CoA thiolase (POT1) [Aspergillus fumigatus Af293] peroxisomal 3-ketoacy-coA thiolase (Kat1) [Aspergillus fumigatus Af293] acetyl-CoA-acetyltransferase [Aspergillus fumigatus Af293]

3-ketoacyl-coA thiolase peroxisomal A precursor [Aspergillus fumigatus Af293] 3-ketoacyl-CoA ketothiolase (Kat1) [Aspergillus fumigatus Af293] (a) peroxisomal 3-ketoacyl-coA thiolase (Kat1) [Aspergillus fumigatus Af293] 3-ketoacyl-coA thiolase peroxisomal A precursor [Aspergillus fumigatus Af293] 3-ketoacyl-CoA ketothiolase (Kat1) [Aspergillus fumigatus Af293] acety-CoA acetyltransferase [Aspergillus fumigatus Af293]

\begin{tabular}{|c|c|c|c|c|}
\hline 89.7 & 89.7 & $96 \%$ & $1.00 E-21$ & $26 \%$ XP_754174.1 \\
\hline 89.4 & 89.4 & $89 \%$ & $2.00 E-21$ & $29 \%$ XP_747673.1 \\
\hline 80.9 & 80.9 & $71 \%$ & $1.00 E-17$ & $31 \%$ XP_753374.1 \\
\hline 7.8 & 67.8 & $78 \%$ & $1.00 E-13$ & $28 \% \mathrm{XP}_{-} 754855.1$ \\
\hline 24 & 224 & $98 \%$ & $2.00 E-69$ & $39 \% \mathrm{xF}$ \\
\hline 221 & 221 & $100 \%$ & $3.00 E-68$ & $37 \%$ XP_751294.1 \\
\hline 212 & 212 & $97 \%$ & $4.00 \mathrm{E}-65$ & $37 \%$ XP 755468.1 \\
\hline 205 & 205 & $97 \%$ & $1.00 E-62$ & $36 \% \times P=751733.1$ \\
\hline 201 & 201 & $98 \%$ & $7.00 \mathrm{E}-61$ & $35 \% \times \mathrm{XP} 752635.1$ \\
\hline 200 & 200 & $98 \%$ & $1.00 E-60$ & $33 \% \times$ ХР 747207.1 \\
\hline
\end{tabular}

$\begin{array}{lllll}270 & 270 & 93 \% & 3.00 E-91 & 56 \% \text { XP_755425.1 } \\ 183 & 183 & 93 \% & 1.00 E-57 & 43 \% \text { XP } 747085.1\end{array}$ $\begin{array}{llllll}137 & 137 & 93 \% & 7.00 E-40 & 33 \% \times P_{-} 748661.1\end{array}$ $\begin{array}{lllll}136 & 136 & 93 \% & 3.00 E-39 & 33 \% \times P_{-} 755398.1 \\ 115 & 115 & 95 \% & 500-31 & 30 \% X_{-} 754471\end{array}$ $\begin{array}{lllll}115 & 115 & 95 \% & 5.00 \mathrm{E}-31 & 30 \% \mathrm{XP}_{-} 754174.1 \\ 106 & 106 & 95 \% & 2.00 \mathrm{-}-27 & 30 \% \mathrm{XP}_{-} 754471.1\end{array}$ $\begin{array}{lllll}92.4 & 92.4 & 94 \% & 2.00 E-22 & 29 \% \text { XP_749938. } \\ 82.8 & 82.8 & 73 \% & 5.00 E-19 & 33 \% \text { XP } 754855.1\end{array}$ $\begin{array}{rrrrr}79 & 79 & 68 \% & 1.00 E-17 & 27 \% \times X_{-}^{-747673.1} \\ 75 & 75.9 & 63 \% & 5.00 E-16 & 33 \% X_{-} 753374.1\end{array}$

$\begin{array}{lllll}213 & 213 & 99 \% & 4.00 E-69 & 46 \% \\ 175 & 175 & 96755425.1\end{array}$ \begin{tabular}{lllll}
175 & 175 & $96 \%$ & $2.000-54$ & $41 \% \times P_{-} 747085.1$ \\
136 & 136 & $98 \%$ & $4.005-39$ & $33 \%$ \\
\hline
\end{tabular} $\begin{array}{llllll}136 & 136 & 98 \% & 4.00 E-39 & 33 \% \times \mathrm{P}_{-} 7555398.1 \\ 134 & 134 & 96 \% & 1.00 E-38 & 33 \% \mathrm{XP}_{-} 748661.1 \\ 112 & 112 & 97 \% & 500-30 & 34 \% \mathrm{XP}_{-} 754471.1\end{array}$

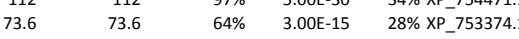

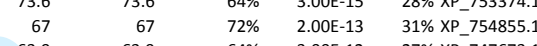
$\begin{array}{lllll}63.9 & 63.9 & 64 \% & 3.00 \mathrm{E}-12 & 27 \% \times \mathrm{XP}_{-} 747673.1\end{array}$ 

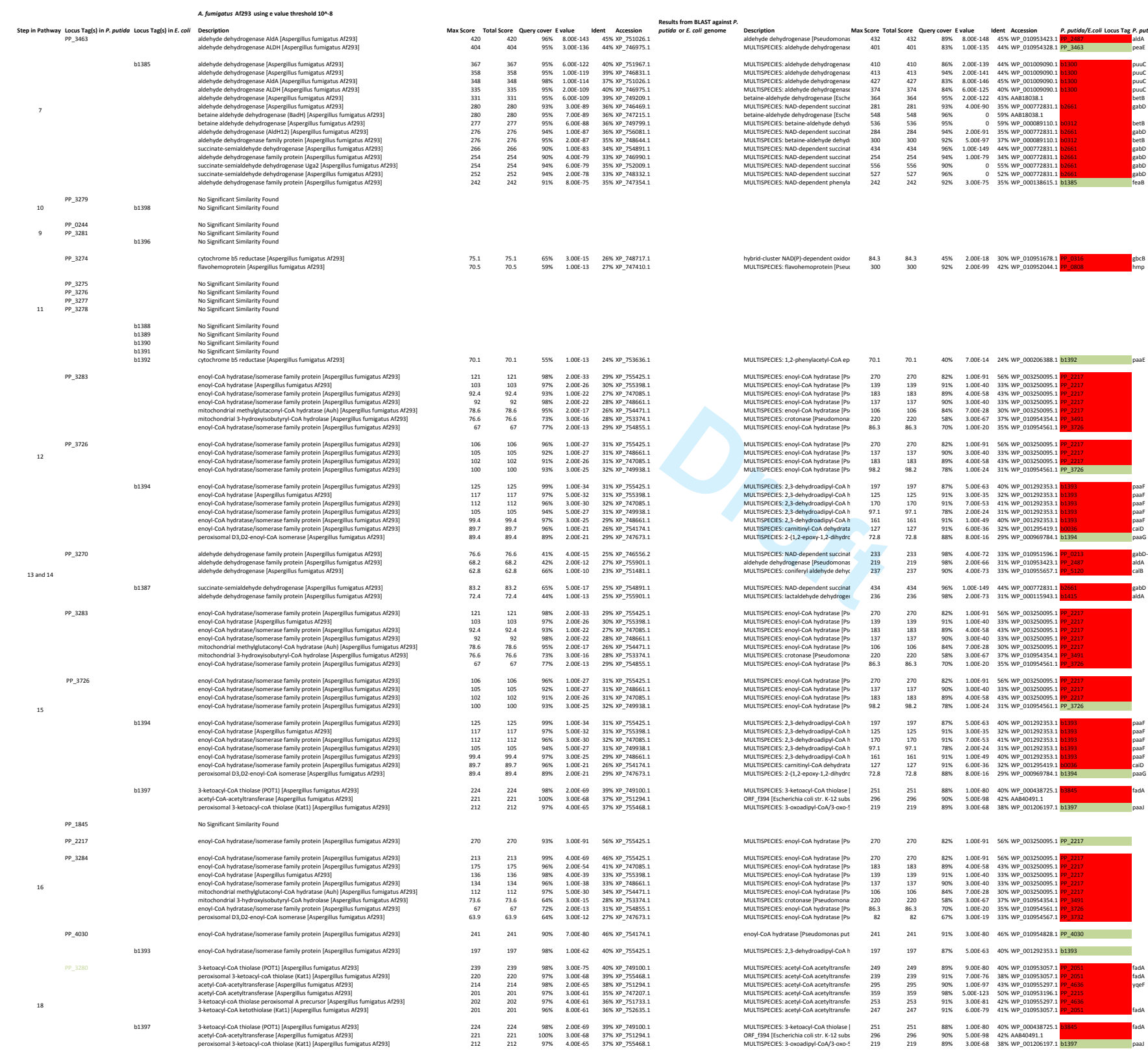

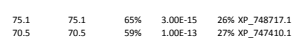

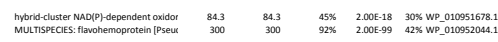
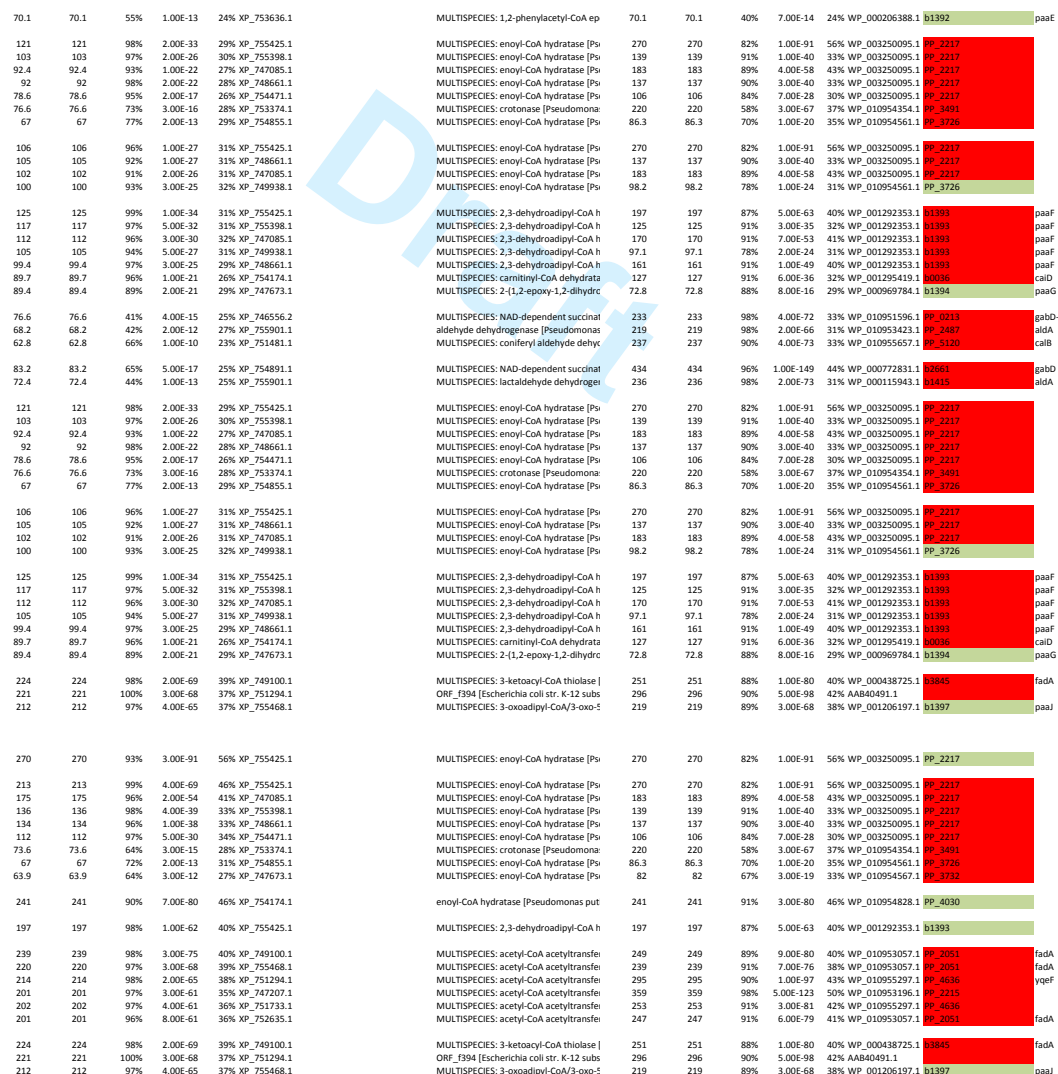